EUROPEAN LABORATORY FOR PARTICLE PHYSICS

CERN-EP/98-054

April 14, 1998

\title{
Photon and Light Meson Production in Hadronic $\mathrm{Z}^{0}$ Decays
}

\author{
The OPAL Collaboration
}

\begin{abstract}

$$
\begin{aligned}
\left\langle n_{\gamma}\right\rangle & =20.97 \pm 0.02 \pm 1.15 \\
\left\langle n_{\pi^{0}}\right\rangle & =9.55 \pm 0.06 \pm 0.75 \\
\left\langle n_{\eta}\right\rangle & =0.97 \pm 0.03 \pm 0.11 \\
\left\langle n_{\rho^{ \pm}}\right\rangle & =2.40 \pm 0.06 \pm 0.43 \\
\left\langle n_{\omega}\right\rangle & =1.04 \pm 0.04 \pm 0.14 \\
\left\langle n_{\eta^{\prime}}\right\rangle & =0.14 \pm 0.01 \pm 0.02 \\
\left\langle n_{\mathrm{a}_{0}^{ \pm}}\right\rangle & =0.27 \pm 0.04 \pm 0.10
\end{aligned}
$$
\end{abstract}

The inclusive production rates and differential cross-sections of photons and mesons with a final state containing photons have been measured with the OPAL detector at LEP. The light mesons covered by the measurements are the $\pi^{0}, \eta, \rho(770)^{ \pm}, \omega(782), \eta^{\prime}(958)$ and $\mathrm{a}_{0}(980)^{ \pm}$. The particle multiplicities per hadronic $\mathrm{Z}^{0}$ decay, extrapolated to the full energy range, are:

where the first errors are statistical and the second systematic. In general, the results are in agreement with the predictions of the JETSET and HERWIG Monte Carlo models.

(Submitted to European Physical Journal, C) 


\section{The OPAL Collaboration}

K. Ackerstaff ${ }^{8}$, G. Alexander ${ }^{23}$, J. Allison ${ }^{16}$, N. Altekamp ${ }^{5}$, K.J. Anderson ${ }^{9}$, S. Anderson ${ }^{12}$, S. Arcelli ${ }^{2}$, S. Asai ${ }^{24}$, S.F. Ashby ${ }^{1}$, D. Axen ${ }^{29}$, G. Azuelos ${ }^{18, a}$, A.H. Ball ${ }^{17}$, E. Barberio ${ }^{8}$, R.J. Barlow ${ }^{16}$, R. Bartoldus ${ }^{3}$, J.R. Batley ${ }^{5}$, S. Baumann ${ }^{3}$, J. Bechtluft ${ }^{14}$, T. Behnke ${ }^{8}$, K.W. Bell ${ }^{20}$, G. Bella ${ }^{23}$, S. Bentvelsen ${ }^{8}$, S. Bethke ${ }^{14}$, S. Betts ${ }^{15}$, O. Biebel ${ }^{14}$, A. Biguzzi ${ }^{5}$,

S.D. Bird ${ }^{16}$, V. Blobel ${ }^{27}$, I.J. Bloodworth ${ }^{1}$, M. Bobinski ${ }^{10}$, P. Bock ${ }^{11}$, J. Böhme ${ }^{14}$,

M. Boutemeur ${ }^{34}$, S. Braibant ${ }^{8}$, P. Bright-Thomas ${ }^{1}$, R.M. Brown ${ }^{20}$, H.J. Burckhart ${ }^{8}$,

C. Burgard ${ }^{8}$, R. Bürgin ${ }^{10}$, P. Capiluppi ${ }^{2}$, R.K. Carnegie $^{6}$, A.A. Carter ${ }^{13}$, J.R. Carter ${ }^{5}$,

C.Y. Chang ${ }^{17}$, D.G. Charlton ${ }^{1, b}$, D. Chrisman ${ }^{4}$, C. Ciocca ${ }^{2}$, P.E.L. Clarke ${ }^{15}$, E. Clay ${ }^{15}$,

I. Cohen ${ }^{23}$, J.E. Conboy ${ }^{15}$, O.C. Cooke ${ }^{8}$, C. Couyoumtzelis ${ }^{13}$, R.L. Coxe ${ }^{9}$, M. Cuffiani ${ }^{2}$, S. Dado ${ }^{22}$, G.M. Dallavalle ${ }^{2}$, R. Davis ${ }^{30}$, S. De Jong ${ }^{12}$, L.A. del Pozo ${ }^{4}$, A. de Roeck ${ }^{8}$, K. Desch ${ }^{8}$,

B. Dienes ${ }^{33, d}$, M.S. Dixit ${ }^{7}$, M. Doucet ${ }^{18}$, J. Dubbert ${ }^{34}$, E. Duchovni ${ }^{26}$, G. Duckeck ${ }^{34}$,

I.P. Duerdoth ${ }^{16}$, D. Eatough ${ }^{16}$, P.G. Estabrooks ${ }^{6}$, E. Etzion ${ }^{23}$, H.G. Evans ${ }^{9}$, F. Fabbri ${ }^{2}$, A. Fanfani ${ }^{2}$, M.Fanti ${ }^{2}$, A.A.Faust ${ }^{30}$, F. Fiedler ${ }^{27}$, M. Fierro ${ }^{2}$, H.M. Fischer ${ }^{3}$, I. Fleck ${ }^{8}$,

R. Folman ${ }^{26}$, A. Fürtjes ${ }^{8}$, D.I. Futyan ${ }^{16}$, P. Gagnon ${ }^{7}$, J.W. Gary ${ }^{4}$, J. Gascon ${ }^{18}$,

S.M. Gascon-Shotkin ${ }^{17}$, C. Geich-Gimbel ${ }^{3}$, T. Geralis ${ }^{20}$, G. Giacomelli ${ }^{2}$, P. Giacomelli ${ }^{2}$,

V. Gibson ${ }^{5}$, W.R. Gibson ${ }^{13}$, D.M. Gingrich ${ }^{30, a}$, D. Glenzinski ${ }^{9}$, J. Goldberg ${ }^{22}$, W. Gorn ${ }^{4}$,

C. Grandi ${ }^{2}$, E. Gross ${ }^{26}$, J. Grunhaus ${ }^{23}$, M. Gruwée ${ }^{27}$, G.G. Hanson ${ }^{12}$, M. Hansroul ${ }^{8}$, M. Hapke ${ }^{13}$,

C.K. Hargrove ${ }^{7}$, C. Hartmann ${ }^{3}$, M. Hauschild ${ }^{8}$, C.M. Hawkes ${ }^{5}$, R. Hawkings ${ }^{27}$,

R.J.Hemingway ${ }^{6}$, M.Herndon ${ }^{17}$, G. Herten ${ }^{10}$, R.D. Heuer ${ }^{8}$, M.D. Hildreth ${ }^{8}$, J.C. Hill ${ }^{5}$,

S.J.Hillier ${ }^{1}$, P.R. Hobson ${ }^{25}$, A. Hocker ${ }^{9}$, R.J.Homer ${ }^{1}$, A.K. Honma ${ }^{28, a}$, D. Horváth ${ }^{32, c}$,

K.R. Hossain ${ }^{30}$, R. Howard ${ }^{29}$, P. Hüntemeyer ${ }^{27}$, P. Igo-Kemenes ${ }^{11}$, D.C. Imrie ${ }^{25}$, K. Ishii ${ }^{24}$,

F.R. Jacob ${ }^{20}$, A. Jawahery ${ }^{17}$, H. Jeremie ${ }^{18}$, M. Jimack ${ }^{1}$, A. Joly ${ }^{18}$, C.R. Jones ${ }^{5}$, P. Jovanovic ${ }^{1}$,

T.R. Junk ${ }^{8}$, D. Karlen ${ }^{6}$, V. Kartvelishvili ${ }^{16}$, K. Kawagoe ${ }^{24}$, T. Kawamoto ${ }^{24}$, P.I. Kayal ${ }^{30}$,

R.K.Keeler ${ }^{28}$, R.G. Kellogg ${ }^{17}$, B.W. Kennedy ${ }^{20}$, A. Klier ${ }^{26}$, S. Kluth ${ }^{8}$, T. Kobayashi ${ }^{24}$,

M. Kobel ${ }^{3, e}$, D.S. Koetke ${ }^{6}$, T.P. Kokott ${ }^{3}$, M. Kolrep ${ }^{10}$, S. Komamiya ${ }^{24}$, R.V. Kowalewski ${ }^{28}$,

T. Kress ${ }^{11}$, P. Krieger ${ }^{6}$, J. von Krogh ${ }^{11}$, P. Kyberd ${ }^{13}$, G.D. Lafferty ${ }^{16}$, D. Lanske ${ }^{14}$, J. Lauber ${ }^{15}$,

S.R. Lautenschlager ${ }^{31}$, I. Lawson ${ }^{28}$, J.G. Layter ${ }^{4}$, D. Lazic ${ }^{22}$, A.M. Lee ${ }^{31}$, E. Lefebvre ${ }^{18}$,

D. Lellouch ${ }^{26}$, J. Letts ${ }^{12}$, L. Levinson ${ }^{26}$, R. Liebisch ${ }^{11}$, B. List ${ }^{8}$, C. Littlewood ${ }^{5}$, A.W. Lloyd ${ }^{1}$,

S.L. Lloyd ${ }^{13}$, F.K. Loebinger ${ }^{16}$, G.D. Long ${ }^{28}$, M.J. Losty ${ }^{7}$, J. Ludwig ${ }^{10}$, D. Lui ${ }^{12}$, A. Macchiolo ${ }^{2}$, A. Macpherson ${ }^{30}$, M. Mannelli ${ }^{8}$, S. Marcellini' ${ }^{2}$, C. Markopoulos ${ }^{13}$, A.J. Martin ${ }^{13}$, J.P. Martin ${ }^{18}$, G. Martinez ${ }^{17}$, T. Mashimo ${ }^{24}$, P. Mättig ${ }^{26}$, W.J. McDonald ${ }^{30}$, J. McKenna ${ }^{29}$, E.A. Mckigney ${ }^{15}$, T.J.McMahon ${ }^{1}$, R.A. McPherson ${ }^{28}$, F. Meijers ${ }^{8}$, S. Menke ${ }^{3}$, F.S. Merritt ${ }^{9}$, H. Mes ${ }^{7}$, J. Meyer ${ }^{27}$, A. Michelini ${ }^{2}$, S. Mihara ${ }^{24}$, G. Mikenberg ${ }^{26}$, D.J.Miller ${ }^{15}$, R. Mir $^{26}$, W. Mohr ${ }^{10}$, A. Montanari ${ }^{2}$,

T. Mori ${ }^{24}$, K. Nagai ${ }^{26}$, I. Nakamura ${ }^{24}$, H.A. Neal ${ }^{12}$, B. Nellen ${ }^{3}$, R. Nisius ${ }^{8}$, S.W. O’Neale ${ }^{1}$,

F.G. Oakham ${ }^{7}$, F. Odorici ${ }^{2}$, H.O. Ogren ${ }^{12}$, M.J. Oreglia ${ }^{9}$, S. Orito ${ }^{24}$, J. Pálinkás ${ }^{33, d}$,

G.Pásztor ${ }^{32}$, J.R. Pater ${ }^{16}$, G.N.Patrick ${ }^{20}$, J.Patt ${ }^{10}$, R. Perez-Ochoa ${ }^{8}$, S. Petzold ${ }^{27}$,

P. Pfeifenschneider ${ }^{14}$, J.E. Pilcher ${ }^{9}$, J. Pinfold ${ }^{30}$, D.E. Plane ${ }^{8}$, P. Poffenberger ${ }^{28}$, B. Poli ${ }^{2}$, J. Polok ${ }^{8}$, M. Przybzien ${ }^{8}$, C. Rembser ${ }^{8}$, H. Rick ${ }^{8}$, S. Robertson ${ }^{28}$, S.A. Robins ${ }^{22}$, N. Rodning ${ }^{30}$, J.M. Roney ${ }^{28}$, K. Roscoe ${ }^{16}$, A.M. Rossi ${ }^{2}$, Y.Rozen ${ }^{22}$, K. Runge ${ }^{10}$, O. Runolfsson ${ }^{8}$, D.R. Rust ${ }^{12}$, K. Sachs ${ }^{10}$, T.Saeki ${ }^{24}$, O.Sahr ${ }^{34}$, W.M.Sang ${ }^{25}$, E.K.G.Sarkisyan ${ }^{23}$, C.Sbarra ${ }^{29}$, A.D.Schaile ${ }^{34}$,

O. Schaile ${ }^{34}$, F. Scharf ${ }^{3}$, P.Scharff-Hansen ${ }^{8}$, J. Schieck ${ }^{11}$, B. Schmitt ${ }^{8}$, S. Schmitt ${ }^{11}$,

A. Schöning ${ }^{8}$, T. Schorner ${ }^{34}$, M. Schröder ${ }^{8}$, M. Schumacher ${ }^{3}$, C. Schwick ${ }^{8}$, W.G. Scott ${ }^{20}$,

R. Seuster ${ }^{14}$, T.G.Shears ${ }^{8}$, B.C. Shen ${ }^{4}$, C.H. Shepherd-Themistocleous ${ }^{8}$, P. Sherwood ${ }^{15}$,

G.P.Siroli ${ }^{2}$, A.Sittler ${ }^{27}$, A.Skuja ${ }^{17}$, A.M.Smith ${ }^{8}$, G.A.Snow ${ }^{17}$, R. Sobie ${ }^{28}$,

S. Söldner-Rembold ${ }^{10}$, M. Sproston ${ }^{20}$, A. Stahl ${ }^{3}$, K. Stephens ${ }^{16}$, J.Steuerer ${ }^{27}$, K. Stoll ${ }^{10}$, 


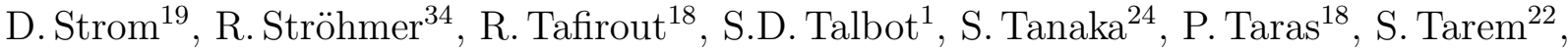
R. Teuscher ${ }^{8}$, M. Thiergen ${ }^{10}$, M.A. Thomson ${ }^{8}$, E. von Törne ${ }^{3}$, E. Torrence $^{8}$, S. Towers ${ }^{6}$, I. Trigger ${ }^{18}$, Z. Trócsányi ${ }^{33}$, E. Tsur ${ }^{23}$, A.S. Turcot ${ }^{9}$, M.F. Turner-Watson ${ }^{8}$, R. Van Kooten ${ }^{12}$, P. Vannerem ${ }^{10}$, M. Verzocchi ${ }^{10}$, P. Vikas ${ }^{18}$, H. Voss ${ }^{3}$, F. Wäckerle ${ }^{10}$, A. Wagner ${ }^{27}$, C.P. Ward ${ }^{5}$, D.R. Ward ${ }^{5}$, P.M. Watkins ${ }^{1}$, A.T. Watson ${ }^{1}$, N.K. Watson ${ }^{1}$, P.S. Wells ${ }^{8}$, N. Wermes ${ }^{3}$, J.S. White ${ }^{28}$, G.W.Wilson ${ }^{14}$, J.A. Wilson ${ }^{1}$, T.R. Wyatt ${ }^{16}$, S. Yamashita ${ }^{24}$, G. Yekutieli26, V. Zacek ${ }^{18}$, D. Zer-Zion ${ }^{8}$

${ }^{1}$ School of Physics and Astronomy, University of Birmingham, Birmingham B15 2TT, UK

${ }^{2}$ Dipartimento di Fisica dell' Università di Bologna and INFN, I-40126 Bologna, Italy

${ }^{3}$ Physikalisches Institut, Universität Bonn, D-53115 Bonn, Germany

${ }^{4}$ Department of Physics, University of California, Riverside CA 92521, USA

${ }^{5}$ Cavendish Laboratory, Cambridge CB3 0HE, UK

${ }^{6}$ Ottawa-Carleton Institute for Physics, Department of Physics, Carleton University, Ottawa, Ontario K1S 5B6, Canada

${ }^{7}$ Centre for Research in Particle Physics, Carleton University, Ottawa, Ontario K1S 5B6, Canada

${ }^{8}$ CERN, European Organisation for Particle Physics, CH-1211 Geneva 23, Switzerland

${ }^{9}$ Enrico Fermi Institute and Department of Physics, University of Chicago, Chicago IL 60637, USA

${ }^{10}$ Fakultät für Physik, Albert Ludwigs Universität, D-79104 Freiburg, Germany

${ }^{11}$ Physikalisches Institut, Universität Heidelberg, D-69120 Heidelberg, Germany

${ }^{12}$ Indiana University, Department of Physics, Swain Hall West 117, Bloomington IN 47405, USA

${ }^{13}$ Queen Mary and Westfield College, University of London, London E1 4NS, UK

${ }^{14}$ Technische Hochschule Aachen, III Physikalisches Institut, Sommerfeldstrasse 26-28, D-52056 Aachen, Germany

${ }^{15}$ University College London, London WC1E 6BT, UK

${ }^{16}$ Department of Physics, Schuster Laboratory, The University, Manchester M13 9PL, UK

${ }^{17}$ Department of Physics, University of Maryland, College Park, MD 20742, USA

${ }^{18}$ Laboratoire de Physique Nucléaire, Université de Montréal, Montréal, Quebec H3C 3J7, Canada

${ }^{19}$ University of Oregon, Department of Physics, Eugene OR 97403, USA

${ }^{20}$ Rutherford Appleton Laboratory, Chilton, Didcot, Oxfordshire OX11 0QX, UK

${ }^{22}$ Department of Physics, Technion-Israel Institute of Technology, Haifa 32000, Israel

${ }^{23}$ Department of Physics and Astronomy, Tel Aviv University, Tel Aviv 69978, Israel

${ }^{24}$ International Centre for Elementary Particle Physics and Department of Physics, University of Tokyo, Tokyo 113, and Kobe University, Kobe 657, Japan

${ }^{25}$ Institute of Physical and Environmental Sciences, Brunel University, Uxbridge, Middlesex UB8 3PH, UK

${ }^{26}$ Particle Physics Department, Weizmann Institute of Science, Rehovot 76100, Israel

${ }^{27}$ Universität Hamburg/DESY, II Institut für Experimental Physik, Notkestrasse 85, D-22607 Hamburg, Germany

${ }^{28}$ University of Victoria, Department of Physics, P O Box 3055, Victoria BC V8W 3P6, Canada

${ }^{29}$ University of British Columbia, Department of Physics, Vancouver BC V6T 1Z1, Canada

${ }^{30}$ University of Alberta, Department of Physics, Edmonton AB T6G 2J1, Canada 
${ }^{31}$ Duke University, Dept of Physics, Durham, NC 27708-0305, USA

${ }^{32}$ Research Institute for Particle and Nuclear Physics, H-1525 Budapest, P O Box 49, Hungary

${ }^{33}$ Institute of Nuclear Research, H-4001 Debrecen, P O Box 51, Hungary

${ }^{34}$ Ludwigs-Maximilians-Universität München, Sektion Physik, Am Coulombwall 1, D-85748

Garching, Germany

${ }^{a}$ and at TRIUMF, Vancouver, Canada V6T 2A3

${ }^{b}$ and Royal Society University Research Fellow

${ }^{c}$ and Institute of Nuclear Research, Debrecen, Hungary

${ }^{d}$ and Department of Experimental Physics, Lajos Kossuth University, Debrecen, Hungary

${ }^{e}$ on leave of absence from the University of Freiburg 


\section{Introduction}

In high-energy collisions, the transition from quarks and gluons to stable hadrons can only be described by phenomenological models [1]. Among the basic features that these hadronisation models attempt to reproduce are the multiplicity and energy spectrum of each hadron species. The large sample of hadronic $\mathrm{Z}^{0}$ decays collected at LEP is ideal to test these models and to improve their accuracy, as the initial state in this process is theoretically well understood. With the versatility of the LEP detectors, these measurements can be extended to most of the low-lying particle states [0 [12].

This paper describes the OPAL measurements of the differential production cross-sections in hadronic $\mathrm{Z}^{0}$ decays of photons and of light mesons decaying to final states containing at least one photon. The mesons studied are the $\pi^{0}, \eta, \rho(770)^{ \pm}, \omega(782), \eta^{\prime}(958)$ and $\mathrm{a}_{0}(980)^{ \pm}$. The measurements require a good understanding of both the detector response to photons and of the environment in which these particles are produced. For this reason, the results obtained with photons detected as energy deposits in the electromagnetic calorimeter and as pairs of tracks from photon conversions to $\mathrm{e}^{+} \mathrm{e}^{-}$in the central drift chamber are first compared and then combined. This comprehensive study of the production of mesons decaying to photons leads to a better understanding of the systematic effects related to photon detection, making possible a reliable measurement of the inclusive production of photons in $Z^{0}$ decays in a wide energy rangef. The production cross-section of each particle is presented as a function of its scaled energy $x_{E}=E_{\text {particle }} / E_{\text {beam }}$ and of $\log \left(1 / x_{p}\right)$, where $x_{p}=p_{\text {particle }} / p_{\text {beam }}$ is the scaled momentum.

Current measurements of photon and $\pi^{0}, \eta, \omega$ and $\eta^{\prime}$ meson production at LEP are limited by experimental systematic errors [6, [9- [2]. Compared to these studies, the present measurements cover a larger fraction of the total rate of these particles, and a number of the sources of systematic error are different. This is the first measurement of the inclusive production of $\rho^{ \pm}$ and $\mathrm{a}_{0}^{ \pm}$in high-energy $\mathrm{e}^{+} \mathrm{e}^{-}$collisions.

The outline of the paper is the following. The OPAL detector is briefly presented, followed by the description of the event selection and simulation. The following three sections describe the three steps in the particle reconstruction. First, photons are detected either as localised energy deposits in the electromagnetic calorimeter or as two tracks from a $\gamma \rightarrow \mathrm{e}^{+} \mathrm{e}^{-}$conversion within the volume of the central drift chambers. In the second step, photons are combined in pairs to form $\pi^{0}$ and $\eta$ meson candidates. In a final step, the $\pi^{0}$ and $\eta$ meson candidates are combined with one charged track or two oppositely charged tracks to reconstruct $\eta \rightarrow \pi^{0} \pi^{+} \pi^{-}$, $\rho^{ \pm} \rightarrow \pi^{0} \pi^{ \pm}, \omega \rightarrow \pi^{0} \pi^{+} \pi^{-}, \eta^{\prime} \rightarrow \eta \pi^{+} \pi^{-}$and $\mathrm{a}_{0}^{ \pm} \rightarrow \eta \pi^{ \pm}$decays. Each step is described, together with the corresponding rate measurements and evaluations of systematic errors. The following section describes the combination of the results for those particles where more than one decay mode is used. The resulting differential cross-sections for photons and light mesons are then compared to the predictions of different models and to other measurements at LEP. This is followed by the conclusion.

\footnotetext{
${ }^{1}$ For an inclusive measurement of prompt photons, excluding hadron decays and initial state radiation, see ref. [13].
} 


\section{The OPAL detector}

The OPAL detector and its performance are described in detail elsewhere [14]. Only detector elements of importance for this analysis are described here. The central tracking system consists of three drift chambers which surround a silicon microvertex detector [15], all within an axial magnetic field of $0.435 \mathrm{~T}$. The silicon microvertex detector has two layers, at radii of 6.1 and $7.5 \mathrm{~cm}$ from the beam axis, with an intrinsic resolution of $5 \mu \mathrm{m}$ in the $r-\phi$ pland ${ }^{2}$. A precision vertex drift chamber with $24 \mathrm{~cm}$ outer radius provides space points with a resolution of about $50 \mu \mathrm{m}$ in $r-\phi$ and $1 \mathrm{~mm}$ in $z$. The jet chamber has an outer radius $185 \mathrm{~cm}$ and provides up to 159 measurements of space points per track, with a resolution in the $r-\phi$ plane of about $130 \mu \mathrm{m}$. The resolution of the $r-\phi$ component of the track momentum $\left(p_{t}\right)$ is $\sigma_{p_{t}} / p_{t} \sim$ $\sqrt{(0.02)^{2}+\left(0.0015 p_{t}\right)^{2}}$, where $p_{t}$ is in $\mathrm{GeV} / c$. In addition, charged particles can be identified by their specific ionisation energy loss $(\mathrm{d} E / \mathrm{d} x)$ [16]. The jet chamber is surrounded by a system of $z$-chambers, thin drift chambers with a resolution of about $300 \mu \mathrm{m}$ in the $z$ coordinate, which serves to improve the determination of $\theta$.

The tracking detectors and the magnet coil are surrounded by electromagnetic and hadronic calorimeters and muon chambers. In this work, the identification of photons is performed within the acceptance of the barrel electromagnetic calorimeter. This consists of a cylindrical array of 9440 lead glass blocks of 24.6 radiation lengths thickness that covers the polar angle range $|\cos \theta|<0.82$. Each block subtends approximately $40 \times 40 \mathrm{mrad}^{2}$. The energy resolution is improved by correcting for losses due to showers initiated in the material in front of the calorimeter. These showers are detected by thin presampler gas detectors covering the front surface of the electromagnetic calorimeter. Time-of-flight scintillators, situated between the magnet coil and the presampler in the polar angle range $|\cos \theta|<0.72$ are also used to detect these showers.

\section{Data selection and event simulation}

This study is based on a sample of 4.1 million hadronic $\mathrm{Z}^{0}$ decays collected by the OPAL detector at LEP at centre-of-mass energies within $\pm 2 \mathrm{GeV}$ of the $\mathrm{Z}^{0}$ peak. The hadronic event selection [17] has an efficiency of $98.4 \pm 0.4 \%$ with a background of less than $0.2 \%$.

The detection efficiencies for the particles under study are evaluated using 6.4 million hadronic $\mathrm{Z}^{0}$ decays simulated using the Monte Carlo programs JETSET 7.3 and 7.4 [18] tuned to reproduce the global features of hadronic events as observed at LEP [19, 20]. Samples generated by the HERWIG 5.9 program [19,21] are also used for comparison. The generated events are passed through a full simulation of the OPAL detector [22] and are subjected to the same event reconstruction and selection as the data.

2 The OPAL coordinate system is defined so that $z$ is the coordinate parallel to the $\mathrm{e}^{-}$beam, $r$ is the coordinate normal to this axis, $\theta$ is the polar angle with respect to $z$ and $\phi$ is the azimuthal angle about the $z$-axis. 


\section{Photons}

\subsection{Photons reconstructed using the electromagnetic calorimeter}

The electromagnetic calorimeter provides the largest part of the photon sample. To resolve a maximum number of photons in the dense environment of hadronic jets, the location and the energy of the electromagnetic showers are obtained from a fit to the energy deposited in the individual lead glass blocks. The fit uses a description of the lateral shower profile as the sum of two exponentials (see for example ref. [23]) and allows a proper treatment of overlapping showers. The fit can also handle the cases where most of the photon energy is in a single block, a common occurrence for photons in the energy range from 0.1 to $0.3 \mathrm{GeV}$.

Not all energy deposits in the electromagnetic calorimeter are due to photons. Many are due to ionisation or to electromagnetic and hadronic showers caused by charged particles. For this reason, a block lying close to the extrapolated path of a charged track is given a small weight in the fit, provided its energy does not significantly exceed the expectation for a hadronic shower.

Photons may lose energy (typically about $0.2 \mathrm{GeV}$ ) by initiating an electromagnetic shower before reaching the calorimeter. The photon energy is therefore corrected using the signal recorded in the presampler. The efficiency for detecting these showers is further increased by also using the presence of signals in the time-of-flight detectors.

A shower is retained as a photon candidate if it has at least $0.1 \mathrm{GeV}$ energy in the lead glass calorimeter and if the energy corrected using the presampler and the time-of-flight detectors is at least $0.15 \mathrm{GeV}$. An acceptance cut of $|\cos \theta|<0.75$ is imposed to improve the homogeneity in the amount of material traversed by the photons before reaching the calorimeter. The momentum direction of the photon is evaluated assuming that it originates from the primary event vertex determined as described in [24].

According to the Monte Carlo simulations, the overall efficiency for photons above $0.15 \mathrm{GeV}$ is $69 \%$ within the acceptance $|\cos \theta|<0.75$. The purity of the sample is $52 \%$, with the most important background being due to energy deposits from charged particles, neutrons and $\mathrm{K}_{\mathrm{L}}^{0}$ mesons. In simulations of hadronic $\mathrm{Z}^{0}$ decays, the photon angular resolution is approximately $10 \mathrm{mrad}$ and the energy resolution varies from $30 \%$ at $0.15 \mathrm{GeV}$ to $8 \%$ at $20 \mathrm{GeV}$.

For the rate determination, it is useful to compare results obtained from samples with different purities. As shown in fig. 1, the purity depends on the photon energy, the energy deposited in its vicinity, its distance to the closest charged track, the shower shape and the quality of the shower fit. The seven variables shown in fig. 11 are combined in one variable $S$ :

$$
S=\frac{1}{N} \sum_{i=1}^{N}\left(1+\exp \left(\left(v_{i}-c_{i}\right) / t_{i}\right)\right)^{-1},
$$

where the index $i$ runs over the $N=7$ variables $v_{i}$ and the parameters $c_{i}$ and $t_{i}$ are chosen such that the power of the variable $S$ to discriminate between signal and background is maximal. This is achieved by minimising the ratio

$$
\mathcal{R}=\frac{\sigma_{s}^{2}+\sigma_{b}^{2}}{\left(\mu_{s}-\mu_{b}\right)^{2}}
$$


where $\mu_{s}$ and $\sigma_{s}$ are the average and rms values of $S$ for the signal and $\mu_{b}$ and $\sigma_{b}$ are the corresponding values for the background. The minimisation is performed using MINUIT [25] and a sample of simulated events. The contributions to $S$ of the input variables are shown as inserts in figs. 17a to 1 $\mathrm{g}$.

The variable $S$ can be interpreted as the output of a simplified artificial neural network, where the number of parameters optimised using a reference sample of simulated events is reduced to two per input variable. In this way, $S$ is forced to depend on the global properties of the input variables, which should reduce problems due to the imperfection of the detector simulation. In the present application, the discrimination losses relative to more complex artificial networks are negligible. In contrast to a likelihood method, correlations between the input variables are partially taken into account by the simultaneous optimisation of all the $c_{i}$ and $t_{i}$ parameters.

The purity of the Monte Carlo photon sample, $P_{\gamma}$, has a smooth dependence on $S$ which is easily parameterized by an analytical function. The distribution of $P_{\gamma}$ is shown in fig. 2. It is well reproduced by the simulation, in particular in the region $P_{\gamma}>0.5$, where the signal is expected to dominate. The variable $P_{\gamma}$ is used for the systematic studies of photon samples of varying purity described in section 4.3.

\subsection{Reconstruction of photon conversions}

According to the Monte Carlo simulation, $7 \%$ of the photons in the angular range $|\cos \theta|<0.75$ convert to $\mathrm{e}^{+} \mathrm{e}^{-}$pairs in the volume of the central tracking chambers. It is useful to compare the results obtained using this sample with those obtained from photons detected in the calorimeter, since they are affected by different systematic uncertainties.

The selection of photon conversions is optimised to have a high efficiency at low momentum and a good angular resolution, and to be insensitive to details of the detector simulation. Conversions are observed as two oppositely charged tracks in the central detector. The two tracks must have impact parameters relative to the primary event vertex greater than $300 \mu \mathrm{m}$ in the $r-\phi$ plane, and at least one track must have space point measurements inside the $z$-chambers. The average of the two points where the tracks are parallel in the $r-\phi$ plane is taken as the point of conversion. The pair topology is required to be consistent with the expectation for a conversion:

- the distance between the two points where the tracks are parallel in the $r-\phi$ plane is required to be less than $1 \mathrm{~cm}$,

- the radial coordinate of the point of conversion, $r$, must be greater than $3 \mathrm{~cm}$,

- the reconstructed photon must have an impact parameter relative to the primary event vertex in the $r-\phi$ plane smaller than $5 \mathrm{~cm}$,

- the absolute value of the difference between $r$ and the radial coordinate of the first space point measurement on either track must be less than $20 \mathrm{~cm}$. 
According to the Monte Carlo, each of these topological cuts removes less than $2 \%$ of the conversion sample. This loose selection is sufficient to obtain a $90 \%$ pure sample for $r>$ $50 \mathrm{~cm}$. For $r<50 \mathrm{~cm}$, the background increases because of the large track density and it is further required that the $\mathrm{d} E / \mathrm{d} x$ measurements of the two tracks each have a probability greater than $1 \%$ for the electron hypothesis. According to the simulation, this cut removes $4 \%$ of the conversions, achieving a purity of $85 \%$ for the entire conversion sample. No further cuts are applied since this purity is sufficient to obtain invariant mass spectra of pairs of photon candidates where the background is dominated by random combinations of genuine photons.

The direction of the photon in $\theta$ is computed from the track parameters with the added constraint that the photon comes from the primary event vertex. The polar angle of the photon direction is required to be in the same fiducial region used for the calorimetric sample $(|\cos \theta|$ $<0.75)$.

The distribution of the radial coordinate $r$ of the conversion points is shown in figure 3 . The Monte Carlo simulation reproduces the overall shape due to the local concentrations of the material in the detector. However, the numbers are not reproduced exactly: for example, in the data $58.0 \%$ of the conversions lie below $r=50 \mathrm{~cm}$, while in the simulation this fraction is $59.2 \%$. Such discrepancies are considered in the following estimation of the systematic errors.

According to the simulation, in the energy range from 0.15 to $20 \mathrm{GeV}$, the angular resolution on the direction of the photon conversions decreases from 10 to $1 \mathrm{mrad}$ in $\phi$ and from 24 to 15 $\operatorname{mrad}$ in $\theta$, and the energy resolution is approximately constant at 4\%. Approximately two thirds of the photon conversions in the fiducial region are reconstructed and selected, corresponding to an average photon efficiency of about $3 \%$.

\subsection{Evaluation of the photon yield}

As the sizes of the photon candidate samples are large, the precision of the measured yields is expected to be limited by systematic uncertainties. It is therefore important to compare the yields derived from samples obtained with different selection procedures, using different Monte Carlo predictions for the photon efficiencies and different methods to subtract the background. Here, the size of the final systematic errors is reduced by incorporating these tests in the determination of the yield itself.

In a first step, the number $n\left(E_{\gamma}, \Delta E_{\gamma}\right)$ of photons per hadronic $\mathrm{Z}^{0}$ decay in an energy bin of width $\Delta E_{\gamma}$ centred at $E_{\gamma}$ is derived using five different data samples (noted by the index $i$ ), three Monte Carlo samples (index $j$ ) and two methods to estimate the background (index $k$ ):

$$
n^{i, j, k}\left(E_{\gamma}, \Delta E_{\gamma}\right)=\frac{N_{\text {candidates }}^{i}\left(E_{\gamma}, \Delta E_{\gamma}\right)-N_{\mathrm{bkg}}^{i, j, k}\left(E_{\gamma}, \Delta E_{\gamma}\right)}{\epsilon^{i, j}\left(E_{\gamma}, \Delta E_{\gamma}\right) N_{\mathrm{Z}^{0}}} .
$$

Here $N_{\text {candidates }}^{i}$ is the total number of photon candidates in the data, $N_{\mathrm{bkg}}^{i, j, k}$ is the predicted number of fake photons, $\epsilon^{i, j}$ is the efficiency for photons in that energy bin, and $N_{\mathrm{Z}^{0}}$ is the number of $\mathrm{Z}^{0}$ decays. In the Monte Carlo, the background is defined as those candidates that are not unambiguously associated to a single photon, or photons coming from bremsstrahlung radiation or decays of particles produced in interactions with the material of the detector. For 
example, the Monte Carlo predicts that about half of the candidates above $15 \mathrm{GeV}$ result from the overlap of the two photons from high-energy $\pi^{0}$ decays. These unresolved photons cannot be counted appropriately and are therefore considered as background.

The five data samples are the conversion sample, three calorimetric samples with requirements $P_{\gamma}>0.0,0.5$ and 0.75 , and the calorimetric sample with each entry weighted by $P_{\gamma}$. The three Monte Carlo samples used for evaluating $N_{\mathrm{bkg}}^{i, j, k}$ and $\epsilon^{i, j}$ are based on the JETSET tunes of ref. [19] and [20], and the HERWIG tune of ref. [19]. The two prescriptions for the determination of $N_{\mathrm{bkg}}^{i, j, k}$ are to take the Monte Carlo prediction and to scale it either according to the number of events or the number of photons. In total, 30 energy-dependent yields $n^{i, j, k}\left(E_{\gamma}, \Delta E_{\gamma}\right)$ are obtained.

In a second step, the central value for $n\left(E_{\gamma}, \Delta E_{\gamma}\right)$ is obtained from a weighted average of the 30 analyses. The average is first performed on the background assumptions $k$, using the quadratic sums of the statistical errors on the data and on the Monte Carlo as weights. This results in averaged yields $n^{i, j}$ and the rms deviation around this mean, $\sigma_{b k g}^{i, j}$, is taken as the systematic error on the choice of background assumptions. This error is added in quadrature to the total error, and the $n^{i, j}$ 's are averaged over the choice of Monte Carlo $j$, using the new total error as a weight. This in turn yields new averages, $n^{i}$, and the error associated with the choice of Monte Carlo $\sigma_{M C}^{i}$, which are again added in quadrature to the total error. Next, the same procedure is applied to the four calorimetric samples, resulting in an average for these samples, $n^{\text {cal }}$, and a systematic error associated with the use of $P_{\gamma}$. In the end, two independent measurements of $n\left(E_{\gamma}, \Delta E_{\gamma}\right)$ are obtained, one from the calorimetric sample, and one from the conversion sample. At the same time, the three systematic errors associated to the variations of $i, j$ and $k$ have been calculated. The weights used to evaluate the average yields are also used to calculate the average error associated to each source, assuming that the samples being combined are fully correlated. For simplicity, this conservative assumption is also applied to the statistical errors on the data and Monte Carlo samples. This is justified because the statistical errors represent a small fraction of the total errors and, in general, there is a large overlap between the samples being combined. A notable exception is the combination of the calorimetric and conversion samples, which is discussed in section 7 .

The averaging procedure is performed separately for each energy bin in order to take into account the variation with energy of the nature and the size of the systematic uncertainties. As a check, the order of the averaging procedure is reversed, and the resulting yields and errors are compared. No significant differences are observed, indicating that the different systematic tests are largely uncorrelated. However, the variations arising from a given test are assumed to be fully correlated from one energy bin to another.

Two corrections are applied to the average yields. The first one is for the difference in energy calibration between the data and the Monte Carlo explained in detail in section 5.2.3. The second accounts for the difference between the data and the Monte Carlo in the number of photons initiating a shower before reaching the calorimeter. A study of the fraction of calorimeter photons with an associated signal in either the presampler or time-of-flight reveals that the Monte Carlo underestimates by $2 \%$ the probability of initiating a shower before reaching the calorimeter. The efficiency is corrected for this effect. It is important only for photons with an energy comparable to the average energy lost before reaching the calorimeter, i.e., about 200 $\mathrm{MeV}$. 
The numbers of photons per hadronic $Z^{0}$ decay in the energy range $x_{E}>0.003$ obtained from the calorimetric $(\gamma)$ and conversion $\left(\gamma_{c}\right)$ measurements are given in table 1 together with the values of each systematic uncertainty. The sources of these uncertainties are:

- The statistical error on the Monte Carlo samples used to calculate the efficiency.

- The variations observed using different Monte Carlo samples, obtained from the averaging procedure.

- The error associated with $P_{\gamma}$, obtained from the averaging procedure.

- The variations observed when using the different background assumptions, obtained from the averaging procedure.

- Some of the background comes from photons produced in interactions with the material of the detector. There are indications that this effect is not exactly reproduced by the Monte Carlo; see for example ref. [2] and the discussion on the electron bremsstrahlung in section 5.2.3. Therefore, half of the Monte Carlo prediction for this background is conservatively taken as an uncertainty.

- As the measured yields depend on the exact energy calibration, the analyses are repeated by shifting the energy scale by $1 \%$ and the difference in the rates is taken as a systematic error. The size of this shift is justified by the calibration studies described in the following section on $\pi^{0}$ and $\eta$ reconstruction.

The variation of the cuts on $P_{\gamma}$ does not cover all sources of systematic uncertainties on the quality of the simulation. The following systematic errors are considered:

- For the calorimeter data, the error labelled simulation in table 1 is the quadratic sum of the uncertainty on the correction of the probability of initiating a shower before reaching the calorimeter and the yield variation observed when the criteria for the association of charged tracks to energy deposits are varied.

- For the conversion data, the error labelled simulation is obtained by removing each of the selection cuts in turn. The differences between the number of accepted tracks in the data and the Monte Carlo obtained when each cut is removed are added in quadrature.

For the calorimetric measurement, the largest source of systematic uncertainty is the difference between the efficiencies derived from the three simulations (table 1). In contrast to ref. [10], where the most important error arises from the choice of using either JETSET or HERWIG for the determination of the efficiencies, here the largest difference is observed between the two samples generated using the JETSET versions tuned in refs. [19] and [20]. The efficiencies obtained with the HERWIG 5.9 sample are consistent those obtained with the first JETSET sample. Notable differences between the two JETSET samples are the inclusion of $L=1$ mesons [19] and consequent changes to the $\omega$ and $\eta^{\prime}$ rates by factors of 1.5 and 4.5 , respectively. These differences affect the photon efficiencies due to the presence of neighbouring particles. Another difference between the two samples is the version of the detector simulation program [22]. 
For the conversions, the largest source of uncertainty is that associated with the simulation of the selection cuts (table 11). The error is $6.8 \%$ over most of the energy range and is dominated by effects related to the inadequacy in describing the distribution of the radial coordinate of the conversions. At the lowest energy it increases to $10 \%$ due to uncertainties in reconstructing tracks with small curvature radii.

As a consistency check, the photon yields obtained from the calorimetric and the conversion sample have been compared in 10 data sub-samples corresponding to different data-taking periods, spanning six years of operation of OPAL at $\mathrm{Z}^{0}$ energies. The calorimeter efficiency varies by less than $1 \%$ and the number of conversions varies with an $\mathrm{rms}$ of $2.6 \%$, well within the systematic errors estimated for those channels.

The differential cross sections of inclusive photon production as a function of $x_{E}$ and $\log \left(1 / x_{p}\right)$ are presented, interpreted and discussed together with those for the light mesons in section 8 .

\section{$5 \quad$ The channels $\pi^{0} \rightarrow \gamma \gamma$ and $\eta \rightarrow \gamma \gamma$}

In this section, $\pi^{0}$ and $\eta$ mesons are reconstructed as pairs of photons. The branching ratios of the decays $\pi^{0} \rightarrow \gamma \gamma$ and $\eta \rightarrow \gamma \gamma$ are $(98.80 \pm 0.03) \%$ and $(39.25 \pm 0.31) \%$, respectively [26]. The numbers of $\pi^{0}$ and $\eta$ mesons in the data and Monte Carlo samples are determined from fits to the invariant mass spectra of the photon pairs. The selection of candidates is presented, followed by a description of the fits to the invariant mass spectra and the determination of the meson yields. As in the photon case, the yields and some systematic errors are obtained by averaging results based on different samples and using various analysis methods.

\section{$5.1 \quad \pi^{0} \rightarrow \gamma \gamma$ and $\eta \rightarrow \gamma \gamma$ selection}

The $\pi^{0}$ and $\eta$ candidates are obtained by combining in turn all pairs of photon candidates. In the $\eta$ selection, the energy of each photon is required to be larger than $0.3 \mathrm{GeV}$. At this stage, the combinatorial background is large, and it is not possible to extract the $\pi^{0}$ and $\eta$ yields from the invariant mass distribution of the photon pairs.

To reduce the background, the probability $P_{\gamma \gamma}$ that a photon pair comes from a $\pi^{0} \rightarrow \gamma \gamma$ decay as a function of a set of input variables is estimated using the same method as for $P_{\gamma}$ in section 4.1. The input variables for each photon are those shown in fig. 1 $1 \mathrm{a}, \mathrm{b}, \mathrm{d}, \mathrm{f}$, and $\mathrm{g}$, together with the opening angle $\theta_{i j}$ of the photon pair, the number of additional photons in cones of opening angle $\theta_{i j}$ around each of the two photons and the helicity angle of the photon calculated in the $\gamma \gamma$ rest frame. The invariant mass of the pair is excluded from the input variables so that it can be used later on for extracting the yields. The same method is applied to the decays involving conversions and the three functions $P_{\gamma \gamma}^{\pi^{0}} \rightarrow \gamma \gamma, P_{\gamma \gamma}^{\pi^{0} \rightarrow \gamma \gamma_{c}}$ and $P_{\gamma \gamma}^{\pi^{0} \rightarrow \gamma_{c} \gamma_{c}}$ are determined separately. Similarly, the equivalent functions for the $\eta, P_{\gamma \gamma}^{\eta \rightarrow \gamma \gamma}, P_{\gamma \gamma}^{\eta} \rightarrow \gamma \gamma_{c}$ and

\footnotetext{
${ }^{3}$ For the conversions, the input variables corresponding to fig. 1 $\mathrm{b}$, d, f, and $\mathrm{g}$ are not relevant and are therefore not used.
} 
$P_{\gamma \gamma}^{\eta \rightarrow \gamma_{c} \gamma_{c}}$, are evaluated separately. The purity of the $\pi^{0}$ Monte Carlo sample is evaluated using a $\Delta m=0.1 \mathrm{GeV} / c^{2}$-wide invariant mass window centred at $m_{0}=0.135 \mathrm{GeV} / c^{2}$. For the $\eta$, the purity of the Monte Carlo sample is evaluated using $\Delta m=0.2 \mathrm{GeV} / c^{2}$ and $m_{0}=0.5475 \mathrm{GeV} / c^{2}$.

The probability that a pair of photons $i, j$ is part of the $\pi^{0}$ or $\eta$ signal depends not only on the $P_{\gamma \gamma}$ value for this pair, but also on the signal probability for any other pair involving either $i$ or $j$. Assuming that $P_{\gamma \gamma}$ is indeed equal to the signal probability, and assuming that the $P_{\gamma \gamma}$ values of all photon pairs in an event are uncorrelated, the probability that two photons $i$ and $j$ are not related to any other photon in the event is:

$$
\mathbb{P}_{\gamma_{i} \gamma_{j}}=\prod_{k \neq i, j}\left[1-G_{i k}\left(m_{i k}\right)\right] \times \prod_{l \neq i, j}\left[1-G_{j l}\left(m_{j l}\right)\right]
$$

Here $m_{i k}$ is the invariant mass of the pair of photons $i$ and $k$, and

$$
G_{i k}\left(m_{i k}\right)=\frac{P_{\gamma_{i} \gamma_{k}}}{P_{\gamma_{i} \gamma_{k}}+\left(1-P_{\gamma_{i} \gamma_{k}}\right) \frac{\sqrt{2 \pi} \sigma}{\Delta m} \exp \left(\frac{1}{2}\left(\frac{m_{i k}-m_{0}}{\sigma}\right)^{2}\right)}
$$

is the $P_{\gamma \gamma}$ variable modified to take into account the mass dependence of the purity of the $\pi^{0}$ and $\eta$ signals, assuming that the invariant mass peaks are normal distributions of width $\sigma$, which are determined from the Monte Carlo. The products in eq. A are performed over all pairs retained by either the $\pi^{0}$ or the $\eta$ selection. If a pair passes both selections, the larger of the $\pi^{0}$ or $\eta$ signal probability is retained. The combined probability that the photons $i$ and $j$ are from the same $\pi^{0}$ or $\eta$ decay and that they do not take part in any other decay is taken as $\tilde{P}_{\gamma_{i} \gamma_{j}}=P_{\gamma_{i} \gamma_{j}} \times \mathbb{P}_{\gamma_{i} \gamma_{j}}$. According to the simulations, cutting on $\tilde{P}_{\gamma \gamma}$ instead of $P_{\gamma \gamma}$ reduces the combinatorial background by approximately $10 \%$ in the case of the $\pi^{0}$ and by as much as $30 \%$ in the case of the $\eta$.

Since the $\pi^{0}$ and $\eta$ yields are determined from fits to the invariant mass spectra, the effect of a cut on $\tilde{P}_{\gamma \gamma}$ on the shape of the invariant mass distributions has to be studied. The value of $\tilde{P}_{\gamma_{i} \gamma_{j}}$ has a monotonic correlation with the invariant mass of the pair $m_{i j}$, which arises primarily from its dependence on the opening angle $\theta_{i j}$ and the number of additional photons in the cones of opening angle $\theta_{i j}$ around $i$ and $j$. The shifts in the position of the $\pi^{0}$ and $\eta$ mass peaks induced by cuts on $\tilde{P}_{\gamma \gamma}$ are of the order of a few $\mathrm{MeV} / \mathrm{c}^{2}$ and are well reproduced by the Monte Carlo. More importantly, it has been verified in the Monte Carlo that the cuts on $\tilde{P}_{\gamma \gamma}$ do not produce a fake $\pi^{0}$ or $\eta$ peak in the invariant mass distribution of the combinatorial background.

Fig. 1 shows the distributions of $\tilde{P}_{\gamma \gamma}$ for all channels and indicates that their shapes are well reproduced by the Monte Carlo. A more detailed discussion of possible differences will follow in section 5.3. The Monte Carlo predictions for the efficiency for detecting $\pi^{0}$ and $\eta$ mesons using cuts of 0.1 and 0.05 on $\tilde{P}_{\gamma \gamma}$, respectively, are shown in fig. 5. The efficiencies include all effects, including the known branching ratios [26], so that the yields obtained from the $\gamma \gamma, \gamma \gamma_{c}$ and $\gamma_{c} \gamma_{c}$ samples can be directly compared.

\subsection{Analysis of the invariant mass spectra of photon pairs}

As in the photon analysis, the determination of the $\pi^{0}$ and $\eta$ yields is repeated using different data samples and analysis procedures, and the results are averaged to obtain the central values. 
Therefore, the fits to the invariant mass spectra are performed separately for the $\pi^{0} \rightarrow \gamma \gamma$, $\pi^{0} \rightarrow \gamma \gamma_{c}, \pi^{0} \rightarrow \gamma_{c} \gamma_{c}, \eta \rightarrow \gamma \gamma$ and $\eta \rightarrow \gamma \gamma_{c}$ samples. The channel $\eta \rightarrow \gamma_{c} \gamma_{c}$ is not used because of low statistics. The fit is systematically repeated using five values of the cut on $\tilde{P}_{\gamma \gamma}$, two parameterizations of the background and two parameterizations of the signal peaks. In addition, three Monte Carlo samples are used for the determination of the $\pi^{0}$ and $\eta$ efficiencies and, for one of the background parameterization methods, the background shape. These variations are described below. They amount to a total of 60 different methods to extract the rates for each channel.

\subsubsection{Cut variations}

The cut on $\tilde{P}_{\gamma \gamma}$ is varied among the values $0.1,0.2,0.3,0.4$ and 0.5 for the $\pi^{0}$ analysis, and

$0.05,0.10,0.20,0.30$ and 0.40 for the $\eta$ analysis. The variations of the $\tilde{P}_{\gamma \gamma}$ cut are chosen so as to result in a change in acceptance of at least a factor two, in order to test how well $\tilde{G}_{\gamma \gamma}(m)$ is simulated by the Monte Carlo, and also to provide a wide variety of background shapes and levels.

\subsubsection{Background parameterization}

Figs. 6 and 0 show the invariant mass spectra for all five channels. The two background estimations are also shown. In the first one, the background shape is taken from a simulation and is normalised to the number of counts outside the signal region. Possible differences between the shape in the data and in the Monte Carlo are taken into account by adding a linear background to the fit. In the second method, the background is fitted using a second-order polynomial. An additional source of background is considered in the region just above the $\eta$ peak. The reflection from $\omega \rightarrow \gamma \pi^{0}$ decays are taken into account using a Gaussian with a normalisation allowed to vary and a mass and width of 730 and $80 \mathrm{MeV} / c^{2}$, respectively. This peak is caused by the kinematic correlation between one of the photons from the $\pi^{0}$ and the direct photon from the $\omega$, and cannot be absorbed by the polynomial background.

The two background parameterizations are complementary; the first one takes into account all the features of the background shape predicted by the simulations, while the second does not depend on the details of the Monte Carlo. The area of the signal peaks obtained with the two methods are in general not identical, as shown in figs. 6 and 7 . However both methods should give the same yields if the efficiency is determined by applying the same parameterization to the Monte Carlo. As a result, the errors due to the background parameterization are smaller than is suggested by the difference between the two background estimates in figs. 6 and 7 . Part of this difference comes from an ambiguity in the definition of the background in the Monte Carlo. In a few percent of the cases, a shower reconstructed in the calorimeter has contributions from more than one incident particle and cannot be clearly associated to any one of them. However, the contribution of a photon to this shower might be important enough that when it is combined with the photon coming from the same $\pi^{0}$ decay, the invariant mass of the pair may be very close to the mass of the $\pi^{0}$. Even though such pairs may produce a small $\pi^{0}$ peak, the first method consider them as being part of the background while the second 
will tend to treat them as part of the signal. The averaging procedure takes into account this ambiguity and the related uncertainty.

\subsubsection{Signal parameterization}

Fig. 8 shows the shape of the mass peak for the samples $\pi^{0} \rightarrow \gamma \gamma, \pi^{0} \rightarrow \gamma \gamma_{c}, \pi^{0} \rightarrow \gamma_{c} \gamma_{c}$ and $\eta \rightarrow \gamma \gamma$ in the data and in the simulation. In all cases the distributions are obtained by subtracting the fitted background from the raw spectrum. In the fits, the shapes are alternatively parameterized either as a Gaussian function, or as the more complex functions described below.

With high statistics samples such as those in fig. 8, the non-Gaussian structure of the signal peaks is apparent. The peaks for the $\pi^{0} \rightarrow \gamma \gamma$ and $\eta \rightarrow \gamma \gamma$ decays are each described by two Gaussians centred at the same mass. The centroid, widths and amplitudes of the two Gaussians are determined independently for the data and the Monte Carlo. The centroids and widths are well described by the simulation (fig. 8a and d). In the case of $\pi^{0} \rightarrow \gamma \gamma_{c}$ and $\pi^{0} \rightarrow \gamma_{c} \gamma_{c}$ decays

(fig. 8b and c), bremsstrahlung from the conversion electrons produces a pronounced tail toward low invariant masses [27]. In the fit, this tail is described by an exponential convoluted with the fitted mass resolution. The amplitude and decay constant are determined independently for the data and the Monte Carlo. In the case of the $\eta \rightarrow \gamma \gamma_{c}$ signal, the tail is neglected because of the low statistics.

The comparison of the centroids of the $\pi^{0}$ and $\eta$ signals in the data with those obtained in the simulations provides an important check of the energy calibration. The corrections for all five channels are of order $1 \%$ over most of the energy range. This sets the scale for the uncertainty on the energy calibration.

The quality of the detector simulation and its impact on the analysis can be assessed by comparing the widths and the tails of the mass peaks in the data and in the Monte Carlo. The Monte Carlo reproduces well the peak shapes (fig. 8), except for the tail toward low masses for combinations involving one or two low-energy conversions. Such tails have been noticed before [27] and they are due mostly to bremsstrahlung of the conversion electrons. The Monte Carlo simulates correctly the slope of these tails, but underestimates their amplitude by as much as a factor of two. The analysis should not be affected by an inadequate modelling of these tails, since they are free parameters of the fit. In addition, the systematic error includes the effect of neglecting them by assuming Gaussian peak shapes, and the effect is further tested in section 7 by comparing the results derived from the $\gamma$ and $\gamma_{c}$ samples, and from the $\gamma \gamma, \gamma \gamma_{c}$ and $\gamma_{c} \gamma_{c}$ samples.

\subsubsection{Monte Carlo simulations}

The three Monte Carlo samples described in section 4.3 are alternatively used to evaluate the $\pi^{0}$ and $\eta$ efficiencies. When the first background parameterization method is used, the shape of background is taken from the corresponding simulation for consistency. 


\subsection{Determination of the $\pi^{0}$ and $\eta$ yields}

The $\pi^{0}$ and $\eta$ yields and their errors are determined separately for the channels $\pi^{0} \rightarrow \gamma \gamma$, $\pi^{0} \rightarrow \gamma \gamma_{c}, \pi^{0} \rightarrow \gamma_{c} \gamma_{c}, \eta \rightarrow \gamma \gamma$ and $\eta \rightarrow \gamma \gamma_{c}$ using the averaging method described in section 4.3 . As the numbers of mesons $N_{\text {candidates }}^{i}$ are obtained from fits to the invariant mass spectra, the background $N_{\mathrm{bkg}}$ entering eq. 33 is the contribution from $\pi^{0}$ produced in interactions with the material of the detector, which is taken from the simulation. As in section 1.3 , the yields are corrected for the difference between the Monte Carlo and the data in the energy calibration and in the probability that a photon initiates a shower before reaching the calorimeter.

The numbers of $\pi^{0}$ and $\eta$ mesons per hadronic $\mathrm{Z}^{0}$ decay in the energy ranges covered by the present measurement are given in tables 2 and 3 together with the values of each of the systematic uncertainties. These are:

- The statistical error on the Monte Carlo samples used to calculate the efficiency.

- The variations observed in the averaging procedure using different Monte Carlo samples.

- The error associated with $\tilde{P}_{\gamma \gamma}$, obtained from the averaging procedure.

- The variations observed in the averaging procedure when using the different background parameterizations.

- The variations observed in the averaging procedure when using the different signal parameterizations.

- The uncertainty associated with the $\pi^{0}$ produced in interactions with the material of the detector, evaluated as in section 1.3 .

- The uncertainty associated with the energy calibration, evaluated as in section 4.3 .

- The first method to evaluate the background requires the definition of a mass range used for the normalisation of the Monte Carlo prediction to the data. This procedure is not exact because of the presence of tails in the invariant mass distributions of the signal. The bias on the yields resulting from the choice of the normalisation range is estimated using the Monte Carlo, and its size is taken as the systematic error associated with this method of evaluating the background.

- The simulation uncertainties not covered by the variations of the cut on $\tilde{P}_{\gamma \gamma}$, i.e., the errors labelled simulation in table 1, propagated to the $\gamma \gamma, \gamma \gamma_{c}$ and $\gamma_{c} \gamma_{c}$ samples according to the number of calorimetric and conversion photons in the pairs.

As a consistency check, the position of the $\pi^{0}$ and $\eta$ mass peaks has been measured in 10 data sub-samples corresponding to different data-taking periods, spanning six years of operation of OPAL at $\mathrm{Z}^{0}$ energies. The energy scale varies by less than the $1 \%$ systematic uncertainty ascribed to it. The fluctuations in the extracted $\pi^{0}$ and $\eta$ yields are of the same size as those observed in section 4.3 for the numbers of calorimetric and conversion photons.

The dominant systematic uncertainties on the $\pi^{0} \rightarrow \gamma \gamma, \pi^{0} \rightarrow \gamma \gamma_{c}$ and $\pi^{0} \rightarrow \gamma_{c} \gamma_{c}$ yields (table 2) are those that also affect the corresponding $\gamma$ and $\gamma_{c}$ measurements (table 1), namely 
the variation of the efficiency in the different Monte Carlos for the calorimetric data, and the simulation uncertainty associated with reconstruction cuts for the conversion. However, the size of these uncertainties are approximately the same as those associated with the fits to the invariant mass distributions.

The largest systematic error for the $\eta \rightarrow \gamma \gamma$ channel comes from the variation of the cut on $\tilde{P}_{\gamma \gamma}$ (table 3). This is due to the difference between the shape of the $\tilde{P}_{\gamma \gamma}$ distributions in the data and in the Monte Carlo, which becomes clearly visible above $\tilde{P}_{\gamma \gamma}>0.5$ in fig $₫ \mathrm{~b}$ b. Several checks on the $\eta$ sample were performed in order to understand the origin of this discrepancy. A possible explanation is that the Monte Carlo simulations underestimate the number of isolated $\eta$ mesons. This was verified with $\eta$ samples selected requiring that there should be no other photons or charged tracks within a cone of half-angle $15^{\circ}$ from the $\eta$. The simulation underestimates the number of these isolated $\eta$ mesons with $x_{E}>0.1$ by a factor $2.07 \pm 0.11$, while the combinatorial background is well reproduced. The same problem affects $\pi^{0}$ mesons, for which this factor is measured to be $1.99 \pm 0.05$. The factors are the same for $\gamma \gamma$ and $\gamma \gamma_{c}$ samples. They are similar to those observed in ref. [28]. Thus, there is clear evidence that the Monte Carlo simulation underestimates significantly the number of very isolated $\pi^{0}$ and $\eta$ mesons. These mesons are systematically associated with large values of $\tilde{P}_{\gamma \gamma}$. Fortunately, they constitute only a few percent of the total sample of identified mesons and their impact on the inclusive rate is very small. However, they are an indication of yet unexplained shortcomings in current Monte Carlo models, and have to be taken into account in the evaluation of the systematic error. In the present analysis, the error associated with the variation of $\tilde{P}_{\gamma \gamma}$ corresponds to a decrease of acceptance by a factor of two and probes the behaviour of mesons with significantly different environments. An additional check is performed in sect 7 by comparing the $\gamma \gamma$ and $\pi^{0} \pi^{+} \pi^{-}$ channels which have different sensitivity to variations of the cut on $\tilde{P}_{\gamma \gamma}$.

The differential cross sections as a function of $x_{E}$ and $\log \left(1 / x_{p}\right)$ are presented, interpreted and discussed together with those for the photons and the other mesons in section 8 .

\section{The decay channels $\pi^{ \pm} \pi^{0}, \pi^{ \pm} \eta, \pi^{+} \pi^{-} \pi^{0}$ and $\pi^{+} \pi^{-} \eta$}

The reconstruction of $\pi^{0}$ and $\eta$ mesons offer the possibility to reconstruct the dominant $\rho^{ \pm} \rightarrow$ $\pi^{0} \pi^{ \pm}$and $\mathrm{a}_{0}^{ \pm} \rightarrow \eta \pi^{ \pm}$decays, the $\eta, \omega \rightarrow \pi^{0} \pi^{+} \pi^{-}$decays with branching ratios of $(23.2 \pm 0.5) \%$ and $(88.8 \pm 0.7) \%$ and the $\eta^{\prime} \rightarrow \eta \pi^{+} \pi^{-}$decay with a branching ratio of $(43.7 \pm 1.5) \%$ [26]. The method to evaluate the meson yields follows closely that used in section 5. Namely, the numbers of mesons are obtained from fits to the invariant mass spectra of the meson decay products and the final results and some systematic errors are calculated by averaging the yields obtained using different data samples and various analysis methods. Two notable changes with respect to section 5 are the slight modification of the $\pi^{0}$ and $\eta$ selection described below and the adaptation of the fit procedure to the properties of the observed signals and backgrounds presented in sections 6.2 to 6.5 .

The decay channels $\pi^{ \pm} \pi^{0}, \pi^{ \pm} \eta, \pi^{+} \pi^{-} \pi^{0}$ and $\pi^{+} \pi^{-} \eta$ are reconstructed by combining the $\pi^{0}$ and $\eta$ candidates with either one charged track or two oppositely charged tracks. The charged tracks are required to have at least 40 measured space-points in the jet chamber, a momentum component perpendicular to the beam axis of at least $0.15 \mathrm{GeV} / c$, an impact 
parameter relative to the primary event vertex of less than $0.5 \mathrm{~cm}$ in the $r-\phi$ plane and 20 $\mathrm{cm}$ along the $z$ direction. In addition, the $\mathrm{d} E / \mathrm{d} x$ measurement must have a probability greater than $1 \%$ for the pion hypothesis. The $\pi^{0}$ and $\eta$ selections are improved by using

$$
\tilde{G}_{\gamma \gamma}(m)=\frac{\tilde{P}_{\gamma \gamma}}{\tilde{P}_{\gamma \gamma}+\left(1-\tilde{P}_{\gamma \gamma}\right) \frac{\sqrt{2 \pi} \sigma}{\Delta m} \exp \left(\frac{1}{2}\left(\frac{m-m_{0}}{\sigma}\right)^{2}\right)}
$$

instead of $\tilde{P}_{\gamma_{i} \gamma_{j}}$ as the selection variable, using the same values of $m_{0}, \sigma$ and $\Delta m$ as in eq. 5 . The $\gamma \gamma, \gamma \gamma_{c}$ and $\gamma_{c} \gamma_{c}$ samples are simply summed because their purities are approximately the same for a given value of $\tilde{G}_{\gamma \gamma}(m)$. The resolution on the $\pi^{0}$ and $\eta$ momenta and energies are improved by constraining their invariant masses to their nominal values [26] using a kinematic fit. The four-momenta of the charged and neutral particles are added, and the invariant mass of the system is evaluated.

\subsection{Determination of the particle yields}

As in the previous section, the determination of the meson yields is repeated using different data samples and analysis procedures and the results are averaged to obtain the central values. Similarly, the fits are systematically repeated using different values of cuts on $\tilde{G}_{\gamma \gamma}(m)$, different parameterizations of both the background and the signal and using different Monte Carlo samples for the determination of the efficiency and, in some cases, the shape of the background. For channels involving a $\pi^{0}$, the cut on $\tilde{G}_{\gamma \gamma}(m)$ is varied among the values $0.1,0.2,0.3,0.5$ and 0.7. For channels involving an $\eta$ in the final state, the cut values are $0.05,0.1,0.2,0.3$ and 0.4. Since the largest deviations in the predicted efficiencies observed in the previous two sections are those obtained when comparing the two JETSET samples, only these two Monte Carlo samples are used in this section. In the following, the variations in the parameterization of the signals and the backgrounds are presented channel by channel.

\subsection{Analysis of the $\pi^{0} \pi^{ \pm}$invariant mass spectra}

Fig. 9 shows the invariant mass spectra of $\pi^{0} \pi^{ \pm}$combinations for the entire energy range for two different values of the cut on $\tilde{G}_{\gamma \gamma}(m)$. The extraction of the $\rho^{ \pm}$yield from the $\pi^{0} \pi^{ \pm}$invariant mass spectra is complicated by the large width of the resonance, by uncertainties regarding its exact shape and by the reflection from $\omega \rightarrow \pi^{0} \pi^{+} \pi^{-}$decays. An additional complication is the presence of partially reconstructed $\rho^{ \pm}$decays, where only the charged pion and one of the photons come from the decay of a $\rho^{ \pm}$meson while the other photon candidate has another origin. It is therefore particularly useful to compare yields obtained using different cuts on $\tilde{G}_{\gamma \gamma}(m)$ since this variation produces significant shifts in the position of the maximum of the background shape (fig. 9) and also changes the relative number of partially reconstructed $\rho^{ \pm}$ decays.

Two methods are used to evaluate the background. In the first, the background shape is taken from a simulation. It is normalised to the number of counts outside the signal region. This method is used to obtain background-subtracted invariant mass spectra. In the second 
method, the background is parameterized as:

$$
f(m)=p_{1}\left(\Delta_{m}\right)^{p_{2}} \times \exp \left(p_{3} \Delta_{m}+p_{4} \Delta_{m}^{2}\right),
$$

where $\Delta_{m}=m-m_{\pi^{0}}-m_{\pi^{ \pm}}, m$ is the invariant mass of the $\pi^{0} \pi^{ \pm}$system and the parameters $p_{1}$ to $p_{4}$ are determined in the fits to the data. A Gaussian representing the reflection from $\omega \rightarrow \pi^{0} \pi^{+} \pi^{-}$decays is added to this shape, with a width fixed to the Monte Carlo prediction. The amplitude and centroid of the Gaussian are free parameters, in order to absorb possible imperfections in the modelling of the background in this region close to the $\pi^{0} \pi^{ \pm}$threshold. The simulation predicts that the reflections from $\eta \rightarrow \pi^{0} \pi^{+} \pi^{-}$and $\mathrm{K}^{*}(892)^{ \pm} \rightarrow \mathrm{K}^{ \pm} \pi^{0}$ decays are small; they are not included in the fit. The background shapes obtained using the two methods are shown in fig. 9. Also shown are the data before and after the subtraction of the average of the two background shapes. The $\rho^{ \pm}$resonance is clearly observed, albeit at a slightly lower mass than in the Monte Carlo.

The shape of the $\rho^{ \pm}$resonance may be more complex than a relativistic Breit-Wigner. This is the case for the $\rho^{0}$ meson [8,29], where the observed deviations from this shape may be due to residual Bose-Einstein correlations, to interference between the amplitudes of the $\rho$ and the $\pi \pi$ background and to interference with the $\omega$ [30]. The first two effects should also affect the $\rho^{ \pm}$. The most apparent sign of this distortion is a shift towards low mass of the maximum of the resonance. For this reason the position of the pole of the resonance is a free parameter in the fits. Following ref. [30], the modification of the $\rho$ shape is taken into account by multiplying the relativistic Breit-Wigner by a factor

$$
I(m, C)=1+C \frac{m_{0}^{2}-m^{2}}{m \Gamma}
$$

where the parameter $C$ is to be determined from the data. The values of $C$ obtained in fits to the data in different energy bins vary between 0.2 and 1.0, corresponding to shifts of -10 to -30 $\mathrm{MeV} / \mathrm{c}^{2}$ in the position of the maximum of the resonance and consistent with the observations of ref. [30] for the $\rho^{0}$.

The $\rho^{ \pm}$yields are evaluated either from the results of fits to the invariant mass spectra or by numerical integration of background-subtracted spectra. In the fits, the resonance is parameterized as a relativistic Breit-Wigner convoluted with the experimental mass resolution and multiplied by the factor $I(m, C)$. The fits are repeated with the parameter $C$ being either fixed to zero or left as a free parameter. In addition, the experimental resolution is either fixed to the Monte Carlo prediction or left as a free parameter. These variations affect the number of mesons contained in the tails of the resonance. To address this problem in a consistent way, the numbers of $\rho^{ \pm}$mesons are obtained by integrating the fitted resonance shapes over the same range used for the integration of the background-subtracted spectra, from 0.39 to $1.15 \mathrm{GeV} / c^{2}$. All numbers in the data are then multiplied by $1.08 \pm 0.03$, which is the average correction needed to extrapolate the fitted shapes to the range from the $\pi^{0} \pi^{ \pm}$threshold up to $1.5 \mathrm{GeV} / c^{2}$. The integration range corresponds to the mass range to which the resonance is truncated in JETSET and therefore the yields extracted from the simulations are not multiplied by this factor. In addition, JETSET uses a non-relativistic Breit-Wigner shape, without any of the correlations or interference effects just discussed and for this reason the shape of the resonance is determined separately for the data and the Monte Carlo samples.

Another important consideration in the fits to the invariant mass spectra is the treatment of partially reconstructed $\rho^{ \pm}$mesons. If a photon related to the $\rho^{ \pm}$decay contains most of the 
energy of the $\pi^{0}$ and is combined with any low-energy photon, the invariant mass distribution of the system will form a broad peak under the signal. The height of this "bump" is correlated with the $\rho^{ \pm}$yield, but it is wider than the peak of fully reconstructed $\rho^{ \pm}$mesons. The influence of partially reconstructed $\rho^{ \pm}$mesons is taken into account in the analysis and in the systematic errors in the following ways. The bias in the extracted yields is evaluated by comparing the results of fits to the invariant mass spectra of Monte Carlo samples where the partially reconstructed $\rho^{ \pm}$are included or not. In addition, the simulation predicts that the importance of partial reconstruction decreases as the cut on $\tilde{G}_{\gamma \gamma}(m)$ (and therefore the quality of the selected photons) increases. The comparison of the rates measured with different cut values is thus an additional test of how well this effect is simulated.

\subsection{Analysis of the $\eta \pi^{ \pm}$invariant mass spectra}

Fig. 10 shows the invariant mass spectra of $\eta \pi^{ \pm}$combinations for the entire energy range for two values of the cut on $\tilde{G}_{\gamma \gamma}(m)$. The analyses of the $\eta \pi^{ \pm}$and $\pi^{0} \pi^{ \pm}$invariant mass spectra are similar. The same parameterization for the combinatorial background is used (eq. 7) and a Gaussian is added to represent the reflection from $\eta^{\prime} \rightarrow \eta \pi^{+} \pi^{-}$decays. However, because of the low statistics the description in the fit of the $\mathrm{a}_{0}^{ \pm}$resonance is not as detailed as for the $\rho^{ \pm}$. The $\mathrm{a}_{0}^{ \pm}$peak is parameterized by a simple Gaussian. The fitted mean and width of the signal peak are $990 \pm 12$ and $51 \pm 9 \mathrm{MeV} / c^{2}$, respectively, in agreement with the nominal mass of $983.5 \pm 0.9 \mathrm{MeV} / c^{2}$ [26], and consistent with the expected range of 50 to $90 \mathrm{MeV} / c^{2}$ for the width of the resonance in the $\eta \pi^{ \pm}$channel [32]. To control possible biases due to the Gaussian assumption, the fitted yields are used in the averaging procedure together with the integrals of background-subtracted spectra in the range from 880 to $1120 \mathrm{MeV} / \mathrm{c}^{2}$, where the background

shape is either taken from the simulation or from a fit of the analytical background shape to the data outside the signal region.

In fig. 10, the data are shown together with two background distributions obtained either by the fit to the data or by scaling the Monte Carlo prediction. Also shown is the data after the average of the two background shapes is subtracted. A peak is observed, with a position and a width comparable to the prediction of the Monte Carlo for the $\mathrm{a}_{0}^{ \pm}$signal. In the JETSET Monte Carlo the $\mathrm{a}_{0}^{ \pm}$resonance is a non-relativistic Breit-Wigner with a pole at $m_{0}=983 \mathrm{MeV} / c^{2}$ and a width $\Gamma=57 \mathrm{MeV} / c^{2}$, the distribution being truncated at $m_{0} \pm 50 \mathrm{MeV} / c^{2}$. Due to the severe truncation and taking into account the experimental mass resolution, the simulated signal distribution shown in fig. 10 resembles closely that of a Gaussian of width $\sigma \sim 32 \mathrm{MeV} / \mathrm{c}^{2}$. According to the simulation, the effect of partially reconstructed $\eta$ mesons is negligible. This is because the $\eta$ selection imposes more stringent requirements on the photon quality than does the $\pi^{0}$ selection.

\subsection{Analysis of the $\pi^{0} \pi^{+} \pi^{-}$invariant mass spectra}

Fig. $11 \mathrm{a}$ and $\mathrm{c}$ show the invariant mass spectra of $\pi^{0} \pi^{+} \pi^{-}$combinations, for the entire energy range, in the region close to the $\eta$ and $\omega$ signals. Compared to the $\pi^{0} \pi^{ \pm}$invariant mass spectra, the $\pi^{0} \pi^{+} \pi^{-}$analysis benefits from the narrow widths of the $\eta$ and $\omega$ states. However, it suffers from the reduced meson rates and the additional combinatorial background. 
In the fits to the invariant mass distributions, the peaks from $\pi^{0} \pi^{+} \pi^{-}$decays of the $\eta$ and the $\omega$ are each well reproduced by two Gaussians sharing the same centroid, their relative widths and areas being determined from the simulation. In the case of the $\omega$, this double-Gaussian also helps to account for the tails of the Breit-Wigner distribution of the resonance. The fitted parameters of the peak are the area, its centroid and its rms width.

The combinatorial backgrounds for the $\eta$ and the $\omega$ are described by a second- and a third-order polynomial, respectively. The shape of the fit component representing partially reconstructed mesons differs from the one used for the $\rho^{ \pm}$. The $\eta$ and $\omega$ momenta are shared amongst three daughters instead of two as in the case of the $\rho^{ \pm}$. Consequently, the cases where all particles except one of the two photons come from the same decay result in a bump with a narrower width, lying systematically at masses above the peak of fully-reconstructed decays. The adopted shape is an exponential tail at high masses convoluted with a Gaussian with a width equal to that of the signal peak. The exponent of the tail and its area relative to the signal peak are taken from the Monte Carlo prediction.

Two tests are performed to verify that the bump due to partially reconstructed decays is properly described by the Monte Carlo. The first is to compare the rates obtained with different cuts on $\tilde{G}_{\gamma \gamma}(m)$. As the $\pi^{0}$ probability increases, the size of the bump relative to the signal decreases. The second test uses the characteristic matrix elements of the $\eta$ and $\omega$ decays to produce invariant mass spectra with almost no background. Within an experimental precision of a few percent [34], the decay transition probabilities $\lambda_{\eta}$ and $\lambda_{\omega}$ are proportional tof:

$$
\begin{aligned}
& \lambda_{\eta} \propto 1-\frac{T_{0}^{*}}{T_{0, \max }^{*}} \\
& \lambda_{\omega} \propto\left|\vec{p}_{-}^{*} \times \vec{p}_{+}^{*}\right|^{2},
\end{aligned}
$$

where $T_{0}^{*}$ is the kinetic energy of the $\pi^{0}$ in the $\pi^{0} \pi^{+} \pi^{-}$rest frame, $T_{0, \text { max }}^{*}$ is its maximum possible value and $\vec{p}_{+}^{*}\left(\vec{p}_{-}^{*}\right)$ is the momentum of the positively (negatively) charged pion in the $\pi^{0} \pi^{+} \pi^{-}$ rest frame. $\lambda_{\eta}$ and $\lambda_{\omega}$ are normalised such that they vary from 0 to 1 . Random combinations of three pions distributed according to phase space result in a flat $\lambda$ distribution. Therefore the ratio of the $\lambda$ distributions for the signal and the combinatorial background should be proportional to $\lambda$. Extracting from the invariant mass spectra the component proportional to $\lambda$, one obtains distributions with the combinatorial background subtracted, and where the shape of the signal and of the bump of partially reconstructed mesons can be evaluated with more precision.

The method to extract the component proportional to $\lambda$ in the data does not depend on its modelling in the Monte Carlo. The $\lambda$ distribution of the candidates is sampled as a function of their invariant mass, $m$. The behaviour of phase space, $F(\lambda, m)$, including acceptance effects, is taken from combinations with invariant masses above and below the mass peak. In the signal region, it is interpolated using a polynomial function. In each invariant mass bin, the data are fitted with two components, one proportional to the phase space behaviour, $F(\lambda, m)$, and the other proportional to $\lambda F(\lambda, m)$. Figs. 11]b and $\mathrm{d}$ show the components proportional to $\lambda F(\lambda, m)$ extracted from the data in figs. 11a and $\mathrm{c}$, respectively. The distribution for the $\omega$ signal is slightly asymmetric due to a small excess at high mass which is explained in the Monte Carlo as the bump of partially reconstructed mesons (dashed line in fig. 11b). Tests

\footnotetext{
${ }^{4}$ As formulated here, $\lambda_{\eta}$ must be multiplied by the $T_{0}^{*}$ phase-space dependence.
} 
performed on the simulations show that the components proportional to $\lambda F(\lambda, m)$ are excellent approximations of the signal distributions. In both data and Monte Carlo, the meson yields obtained from fits to the total invariant mass distribution or to the component proportional to $\lambda$ agree within $3 \%$.

In JETSET, the matrix element of the decay $\eta \rightarrow \pi^{0} \pi^{+} \pi^{-}$is not simulated. To reproduce the data, the signal events in the Monte Carlo are weighted by a factor 9 of $2 \lambda_{\eta}$. Due to the strong correlation of $\lambda_{\eta}$ with the $\pi^{0}$ energy, this correction changes the total efficiency for the detection of $\eta \rightarrow \pi^{0} \pi^{+} \pi^{-}$decays by as much as $20 \%$.

\subsection{Analysis of the $\eta \pi^{+} \pi^{-}$invariant mass spectra}

Fits to the $\eta \pi^{+} \pi^{-}$invariant mass spectra for the entire energy range are shown in fig. 12, for the data and the Monte Carlo. Given the small statistics, a Gaussian is found to describe adequately the $\eta^{\prime}$ signal. As for the $\mathrm{a}_{0}^{ \pm}$, the contribution from partially reconstructed $\eta$ decays does not need to be parameterized in the fit. In contrast to the three-body decays of the $\eta$ and $\omega$, the Dalitz plot for the decay $\eta^{\prime} \rightarrow \eta \pi^{+} \pi^{-}$is closer to phase space [35] and the matrix element of the decay cannot be used to obtain background-free distributions.

\subsection{Determination of the meson yields}

The $\rho^{ \pm}, \mathrm{a}_{0}^{ \pm}, \eta, \omega$ and $\eta^{\prime}$ yields and their systematic errors are determined using the same averaging method as in sections 4.3 and 5.3. The yields are corrected for the known branching ratios of the different decay modes [26], except for $\mathrm{a}_{0}^{ \pm} \rightarrow \eta \pi^{ \pm}$, where a branching ratio of $90 \pm 10 \%$ is assumed [26, 33].

The numbers of mesons per hadronic $Z^{0}$ decay in the energy ranges covered by the present measurement are given in table 1 together with the values of each systematic uncertainty. These are:

- The statistical error on the Monte Carlo samples used to calculate the efficiency.

- The variations observed using different Monte Carlo samples, obtained from the averaging procedure.

- The error associated with $\tilde{G}_{\gamma \gamma}(m)$, obtained from the averaging procedure.

- The variations observed when using the different background parameterizations, obtained from the averaging procedure. In the case of $\pi^{0} \pi^{+} \pi^{-}$decays, this includes the variations observed when the matrix elements $\lambda_{\eta}$ and $\lambda_{\omega}$ are used.

- The variations observed when the mass resolution is either fixed to the Monte Carlo prediction or left as a free parameter. In the case of the $\rho^{ \pm}$, this also includes the variations observed when $C$ is either fixed to zero or left as a free parameter.

\footnotetext{
${ }^{5}$ The normalisation is chosen such that the integral of the signal, $\int_{0}^{1} F(\lambda, m) d \lambda$, is 1 .
} 
- For the $\rho^{ \pm}$, the error associated with the extrapolation of the resonance beyond the mass range described in section 6.2 .

- The simulation uncertainties not covered by the variations of the cut on $\tilde{G}_{\gamma \gamma}(m)$. The contribution of the $\pi^{0}$ and $\eta$ are the simulation errors quoted in tables 2 and 3 . These are added in quadrature and correspond to a $2 \%$ uncertainty on the charged pion selection.

- The uncertainty due to partially reconstructed mesons, evaluated with the Monte Carlo by comparing the results of fits to mass distributions with and without their contribution. The yield difference is taken as a systematic error.

- In the case of the decays $\eta \rightarrow \pi^{0} \pi^{+} \pi^{-}, \eta^{\prime} \rightarrow \eta \pi^{+} \pi^{-}$and $\mathrm{a}_{0}^{ \pm} \rightarrow \eta \pi^{ \pm}$, the uncertainty on the branching ratio is greater than $1 \%$, and is therefore included.

The differential cross sections as a function of $x_{E}$ and $\log \left(1 / x_{p}\right)$ are presented, interpreted and discussed together with those for the photons and the other mesons in section 8 .

\section{Combination of channels}

The calorimeter and conversion data are compared. The ratio of the total photon rates obtained using identified photon conversions and the calorimeter data is $1.010 \pm 0.002$ (stat.), in agreement well within the size of the systematic errors on the two samples $(4.7 \%$ and $8.3 \%$; see table (1). Comparison of the $\pi^{0}$ rates measured in the energy range common to the three channels $\gamma \gamma, \gamma \gamma_{c}$ and $\gamma_{c} \gamma_{c}$ also yields ratios consistent with one. The ratios of the rates $\gamma \gamma_{c} / \gamma \gamma$ and $\gamma_{c} \gamma_{c} / \gamma \gamma$ are $0.97 \pm 0.02$ (stat.) and $0.96 \pm 0.05$ (stat.), respectively, in perfect agreement given the estimated $8.4 \%, 12.2 \%$ and $23.6 \%$ errors on the rates for the $\gamma \gamma, \gamma \gamma_{c}$ and $\gamma_{c} \gamma_{c}$ channels, respectively (table 2). The agreement is also excellent for the $\gamma \gamma_{c}$ and $\gamma \gamma$ channels for the $\eta$, despite a $34.3 \%$ uncertainty on the $\gamma \gamma_{c}$ channel (table 3).

Given the positive result of these consistency tests, a weighted average of the differential cross-sections measured using the different channels is performed. The weights are taken as the inverse of the square of the total errors. The systematic errors on the combined results are obtained by assuming that the individual systematic errors are completely correlated. In the few cases where the individual measurements and their errors are more than one standard deviation away from the averaged result, the error on the average is scaled using the method of ref. [26].

The $\eta$ differential cross section measurements based on the $\gamma \gamma\left(\gamma \gamma_{c}\right)$ and $\pi^{0} \pi^{+} \pi^{-}$channels have comparable total errors (13.9\% and $12.9 \%$, respectively; see tables 3 and 1 ) and the systematic uncertainties are largely uncorrelated. The two measurements are combined assuming that the errors associated to the track and cluster simulation and to the variations of $\tilde{G}_{\gamma \gamma}(m)$ and of the Monte Carlo are entirely correlated and that all other sources of uncertainty are uncorrelated. In the energy range where both types of data are available, the ratio of the $\pi^{0} \pi^{+} \pi^{-}$to the $\gamma \gamma\left(\gamma \gamma_{c}\right)$ results is constant and equal to $1.14 \pm 0.07$ (stat.) \pm 0.13 (syst.). With the improved knowledge of the absolute normalisation provided by the combination of the two data sets, the error on the total $\eta$ rate is $10.9 \%$. 


\section{Results}

In this section, the experimental meson rates are presented and compared to the predictions of the JETSET and HERWIG Monte Carlo models. To simplify the comparison, the predictions of the default versions of JETSET 7.4 and HERWIG 5.9 are used here, except for the $\mathrm{a}_{0}^{ \pm}$which is not produced in the default version of JETSET 7.4 and for which the prediction of the JETSET version of ref. [19] is used. Two aspects of the model predictions are investigated: the shape of the momentum spectra and the integrated rates.

\subsection{Differential cross-sections}

The differential cross-sections as a function of $x_{E}$ and $\log \left(1 / x_{p}\right)$ of the photon and the $\pi^{0}, \eta$, $\rho^{ \pm}, \omega, \eta^{\prime}$ and $\mathrm{a}_{0}^{ \pm}$mesons are obtained by dividing the yields by the corresponding bin widths. For the $\rho^{ \pm}$and $\mathrm{a}_{0}^{ \pm}$resonances, the relationship between the meson energy and its momentum varies from event to event. The yields are thus evaluated separately in bins of $x_{E}$ and in bins of $\log \left(1 / x_{p}\right)$. The differential cross-sections are listed in tables 5 to 11 together with the statistical and systematic errors. In fig. 13, the data are compared to the JETSET 7.4 predictions normalised to the measured rate. In the simulation, the difference between the slopes of the photon and $\pi^{0}$ distributions (fig. 13a) is strongly constrained by the fact that $92 \%$ of the photons come from $\pi^{0}$ decays. The measured shapes are consistent with the photon and $\pi^{0}$ predictions. The $\rho^{ \pm}$and $\omega$ mesons, both vector particles with similar masses, have similar slopes (fig. 13b) that are also well reproduced by the Monte Carlo. However the predicted $\eta$ spectrum is too soft compared to the measurement (fig. 13. c), while the $\eta^{\prime}$ prediction is consistent with the data within errors. The slope of the $\mathrm{a}_{0}^{ \pm}$distribution (fig. 13d) is well reproduced by JETSET with the parameters of ref. [19].

To emphasise the low-momentum portions of the spectra which represent the largest fraction of the inclusive rates, the differential cross-sections are presented in fig. 14 as a function of $\log \left(1 / x_{p}\right)$. In this figure, the full and dashed curves are the absolute predictions of default JETSET 7.4 and HERWIG 5.9, respectively, except for the $\mathrm{a}_{0}^{ \pm}$prediction of JETSET, taken from ref. 19. JETSET 7.4 reproduces the photon and $\pi^{0}$ data slightly better than HERWIG 5.9. The predictions for the $\mathrm{a}_{0}^{ \pm}$are quite similar, but HERWIG 5.9 provides a better description of the $\rho^{ \pm}, \omega$ and $\eta^{\prime}$ data. However, the $\eta$ momentum spectra predicted by both models are too soft.

\subsection{Maxima of the $\log \left(1 / x_{p}\right)$ distributions}

The location of the maximum of the $\log \left(1 / x_{p}\right)$ distribution is expected to be correlated with the mass of the particle [36]. In addition, its value provides a quantitative measurement of the hardness of the momentum spectrum. The value is extracted by fitting a Gaussian to the data close to the maximum of the distribution. These values are listed in table 12, and shown in fig. 15a where they are compared to other measurements at LEP and to the predictions of JETSET and HERWIG. The errors quoted in the table are the sum of the fit errors and of the uncertainties due to variations of the range of the Gaussian fits. 
As can be seen in fig. 15a, both JETSET and HERWIG reproduce the data within errors, except for the $\eta$, for which the maximum is at a higher value in both JETSET and HERWIG. This confirms that the $\eta$ spectrum in the two models is too soft as was already noted in section 8.1. The OPAL results agree with those of DELPHI [9] and L3 [10]12].

\subsection{Rates extrapolated to $0<x_{E}<1$}

The extrapolation of the rates to the full range of $x_{E}$ is done using the fractions of the rate lying outside the measured range predicted by JETSET 7.4, HERWIG 5.9 and a Gaussian fit to the $\log \left(1 / x_{p}\right)$ distributions. The applied corrections correspond to the average of the lowest and highest values and the maximum deviation is taken as the systematic error on the procedure. The data used to evaluate the extrapolation factors and the associated errors are listed in table 13. The results of the Gaussian fits are considered because of their good description of the data within errors and because in at least one case (the $\eta$ ) it appears that the JETSET and HERWIG shapes may not be appropriate. Other shapes that describe the data equally well are also considered. For the $\pi^{0}$ and the $\eta$ they are, respectively, the $\pi^{ \pm}$and $K_{S}^{0}$ distributions measured by OPAL [2, 3]. The extrapolation factors derived from these experimental shapes are all within the range covered by the predictions of JETSET, HERWIG and the Gaussian fit.

The particle multiplicities per hadronic $\mathrm{Z}^{0}$ decay extrapolated to the full energy range are:

$$
\begin{aligned}
\left\langle n_{\gamma}\right\rangle & =20.97 \pm 0.02 \pm 1.07 \pm 0.42 \\
\left\langle n_{\pi^{0}}\right\rangle & =9.55 \pm 0.06 \pm 0.72 \pm 0.21 \\
\left\langle n_{\eta}\right\rangle & =0.97 \pm 0.03 \pm 0.10 \pm 0.04 \\
\left\langle n_{\rho^{ \pm}}\right\rangle & =2.40 \pm 0.06 \pm 0.43 \pm 0.02 \\
\left\langle n_{\omega}\right\rangle & =1.04 \pm 0.04 \pm 0.13 \pm 0.03 \\
\left\langle n_{\eta^{\prime}}\right\rangle & =0.14 \pm 0.01 \pm 0.02 \pm 0.01 \\
\left\langle n_{\mathrm{a}_{0}^{ \pm}}\right\rangle & =0.27 \pm 0.04 \pm 0.10 \pm 0.01
\end{aligned}
$$

where the first errors are statistical, the second systematic and the third are from the extrapolation procedure.

\subsection{Discussion of the rates and comparison with models}

In table 14, the measured rates are compared to those from other LEP experiments and to the predictions of JETSET 7.4 and HERWIG 5.9. The ratio of the measured rates to the JETSET 7.4 predictions are shown in fig. 15b together with the results from other LEP experiments [6, 9 12. In this figure, the results obtained by ALEPH in limited energy ranges for the photon, $\eta$ and $\eta^{\prime}$ are divided by the JETSET 7.4 predictions in the corresponding range. All the measurements are consistent with each other, except perhaps for the $\eta^{\prime}$ where the measured rate agrees with the ALEPH result [6], but is more than two standard deviations away from that of L3 [12].

According to JETSET 7.4, 97.0\% of all photons come from $\pi^{0}, \eta, \omega$ and $\eta^{\prime}$ decays. The prediction of HERWIG 5.9 is $96.0 \%$. In comparison, the sum of the measured $\pi^{0}, \eta, \omega$ and $\eta^{\prime}$ rates 
multiplied by the known photon multiplicities in their decays [26] accounts for $(95 \pm 5) \%$ of the measured number of photons 5 . The error on the ratio is calculated assuming that the errors on the photon, $\pi^{0}$ and $\eta$ rates are fully correlated except for those associated with the background subtraction and the fit to the invariant mass spectra?. This good agreement provides further evidence that the models offer a reasonable description of the inclusive production of photons in $\mathrm{Z}^{0}$ decays, an assumption on which the measurement of the photon rates relies.

The production rates of the $\pi^{0}$ and $\rho^{ \pm}$can be compared with those of their isospin parters. Using the measurements for the $\pi^{ \pm}$[2] and the $\rho^{0}$ [6, 8], the following ratios of rates are obtained:

$$
\begin{aligned}
2\left\langle n_{\pi^{0}}\right\rangle /\left\langle n_{\pi^{ \pm}}\right\rangle & =1.12 \pm 0.01 \pm 0.08, \\
2\left\langle n_{\rho^{0}}\right\rangle /\left\langle n_{\rho^{ \pm}}\right\rangle & =1.08 \pm 0.04 \pm 0.20,
\end{aligned}
$$

where the first errors are statistical and the second systematic. These ratios are consistent with the predictions of JETSET 7.4 (1.132 and 1.064, respectively) and HERWIG 5.9 (1.147 and 1.032). In these models, most of the deviation from unity comes from the decays $\eta \rightarrow \pi^{0} \pi^{0} \pi^{0}$ and $\eta^{\prime} \rightarrow \rho^{0} \gamma$.

As seen in fig. 15b, the measured rates are consistent with the predictions of JETSET 7.4 and HERWIG 5.9, except for the $\omega$ and the $\eta^{\prime}$ for which the rates are more than two standard deviations smaller than the JETSET 7.4 prediction. The failure of JETSET to reproduce the $\eta^{\prime}$ rate is well understood, as it assumes a similar strange quark content for the $\eta$ and $\eta^{\prime}$ and neglects the effect of their difference in mass on their relative production rate. For this reason, the suppression of the $\eta^{\prime}$ relative to the $\eta$ is a free parameter in JETSET 7.4. The present data suggest that the current suppression factor of 0.4 should be further reduced. In contrast to the $\eta^{\prime}$, no single parameter can modify the $\omega$ rate in JETSET independently of all other mesons. In that model an increase of the $\omega$ rate is necessarily accompanied by an equivalent increase of the $\rho^{0}, \rho^{+}$and $\rho^{-}$rates because, with ideal mixing, these mesons are the corresponding isospin $I=0$ and $I=1$ states. Indeed, the measured $\rho^{ \pm}$and $\omega$ rates (table 14) are consistent with $I=0 / I=1$ symmetry, albeit with a large error. However that symmetry can be broken by cascade decays of heavier mesons such as the $L=1$ states. The experimental information on the production of these states is limited and the present $\mathrm{a}_{0}^{ \pm}$data is interesting in this respect.

Table 14 shows that the $\mathrm{a}_{0}^{+}$and $\mathrm{a}_{0}^{-}$are produced at rates comparable to the $\eta^{\prime}$, a meson of equal spin and similar mass. However, the strangeness content of the $\mathrm{a}_{0}^{ \pm}$and $\eta^{\prime}$ are not expected to be the same. A more relevant comparison is with the $\mathrm{f}_{0}(980)$ meson, which is the isospin $I=0$ partner of the $\mathrm{a}_{0}^{ \pm}$according to the quark model of mesons. The ratio of the rates of the $\mathrm{a}_{0}^{ \pm}$and the $\mathrm{f}_{0}(980)$ [5] is $1.9 \pm 0.8$, compatible with the expected value of 2 .

In the HERWIG cluster fragmentation model [21, the relative production rates of lightflavour mesons are mostly determined by their masses, which affects the phase space available for the cluster decay. This simple ansatz appears to be able to reproduce the measured $\mathrm{a}_{0}^{ \pm}$ rate. The $\mathrm{a}_{0}^{ \pm}$is not present in the default version of JETSET. The inclusion of $L=1$ meson production in hadronisation requires the tuning of additional parameters. The predictions shown in fig. 15 correspond to the choice of parameters of ref. 19 optimised, in part, to

${ }^{6}$ In the data, the $\pi^{0}$ and $\eta$ decays alone account for approximately $91 \%$ and $4 \%$ of all observed photons, respectively, in good agreement with the JETSET 7.4 predictions of $91.9 \%$ and $4.0 \%$.

7These are the errors labelled as "Background subtraction", "Gaussian peaks" and "Background normalisation range" in tables 1 to 3 . The contributions of the $\omega$ and the $\eta^{\prime}$ to the total error are negligible. 
reproduce the available data on $\mathrm{D}$ and $\mathrm{B}$ mesons. The agreement for the $\mathrm{a}_{0}^{ \pm}$may be accidental since the parameters of ref. [19] also predict a substantial $b_{1}(1232)^{ \pm, 0}$ rate of 0.92 meson per $\mathrm{Z}^{0}$ decays. The present $\omega$ and $\rho^{ \pm}$data do not support this prediction. With the $\mathrm{b}_{1}(1232)^{ \pm, 0}$ decaying exclusively to $\omega \pi$, the parameters of ref. [19] result in an $\omega$ rate which exceeds that of its $I=1$ partner, the $\rho$, with $\left\langle n_{\omega}\right\rangle-\left\langle n_{\rho^{ \pm}}\right\rangle / 2=0.56$. The measured difference is $-0.17 \pm 0.26$, in better agreement with the prediction of -0.06 of default JETSET 7.4 with no $L=1$ mesons, and with the prediction of 0.00 of HERWIG 5.9 , with a $b_{1}(1232)^{ \pm, 0}$ rate of 0.32 . Thus, despite the lack of direct measurements for several $L=1$ meson states, it appears possible to constrain the JETSET model by using the available data on all other mesons.

\section{Conclusion}

The inclusive particle multiplicity per hadronic $\mathrm{Z}^{0}$ decay and the differential cross-section have been measured for photons and for $\pi^{0}, \eta, \rho^{ \pm}, \omega, \eta^{\prime}$ and $\mathrm{a}_{0}^{ \pm}$mesons. The $\mathrm{a}_{0}^{ \pm}$is observed for the first time in high-energy $\mathrm{e}^{+} \mathrm{e}^{-}$collisions. It is produced at a rate comparable to that of mesons with a similar mass, such as the $\eta^{\prime}$ and the $f_{0}(980)$. The inclusive $\rho^{ \pm}$production is measured for the first time in hadronic $\mathrm{Z}^{0}$ decays. The models JETSET 7.4 and HERWIG 5.9 with their default parameters reproduce the shape of the measured differential cross-sections, with the exception of that of the $\eta$ meson which is too soft in both models. The absolute rates in HERWIG 5.9 are in good agreement. In JETSET 7.4, the production rates of the $\omega$ and $\eta$ are overestimated by $20 \%$ and $50 \%$, respectively. The present $\mathrm{a}_{0}^{ \pm}$data is a valuable input for the determination of the parameters required for the inclusion of $L=1$ mesons in JETSET. These parameters are further constrained by the data on other mesons like the $\omega$ and the $\rho^{ \pm}$. 


\section{Acknowledgements}

We particularly wish to thank the SL Division for the efficient operation of the LEP accelerator at all energies and for their continuing close cooperation with our experimental group. We thank our colleagues from CEA, DAPNIA/SPP, CE-Saclay for their efforts over the years on the time-of-flight and trigger systems which we continue to use. In addition to the support staff at our own institutions we are pleased to acknowledge the

Department of Energy, USA, National Science Foundation, USA, Particle Physics and Astronomy Research Council, UK, Natural Sciences and Engineering Research Council, Canada, Israel Science Foundation, administered by the Israel Academy of Science and Humanities, Minerva Gesellschaft, Benoziyo Center for High Energy Physics,

Japanese Ministry of Education, Science and Culture (the Monbusho) and a grant under the Monbusho International Science Research Program, German Israeli Bi-national Science Foundation (GIF), Bundesministerium für Bildung, Wissenschaft, Forschung und Technologie, Germany, National Research Council of Canada, Research Corporation, USA, Hungarian Foundation for Scientific Research, OTKA T-016660, T023793 and OTKA F-023259. 


\section{References}

[1] For example: B. Anderson et al, Phys. Rep. 87 (1983) 31;

B.R. Webber, Nucl. Phys. B 238 (1984) 492.

[2] OPAL Collaboration, R. Akers et al., Z. Phys. C 63 (1994) 181.

[3] OPAL Collaboration, R. Akers et al., Z. Phys. C 67 (1995) 389.

[4] OPAL Collaboration, R. Akers et al., Z. Phys. C 68 (1995) 1.

[5] The OPAL Collaboration, K. Ackerstaff et al., Production of $f_{0}(980), f_{2}(1270)$ and $\phi(1020)$ in hadronic $\mathrm{Z}^{0}$ decays., CERN-PPE/98-010, submitted to European Physical Journal, C.

[6] ALEPH Collaboration, R. Barate et al., Phys. Rep. 294 (1998) 1.

[7] DELPHI Collaboration, P. Abreu et al., Nucl. Phys. B 444 (1995) 3;

DELPHI Collaboration, P. Abreu et al., Z. Phys. C 73 (1996) 61;

DELPHI Collaboration, P. Abreu et al., Phys. Lett. B 379 (1996) 309.

[8] DELPHI Collaboration, P. Abreu et al., Z. Phys. C 65 (1995) 587.

[9] DELPHI Collaboration, W. Adam et al., Z. Phys. C 69 (1996) 561.

[10] L3 Collaboration, M. Acciarri et al., Phys. Lett. B 328 (1994) 223.

[11] L3 Collaboration, M. Acciarri et al., Phys. Lett. B 371 (1996) 126.

[12] L3 Collaboration, M. Acciarri et al., Phys. Lett. B 393 (1997) 465.

[13] OPAL Collaboration, K. Ackerstaff et al., European Physical Journal C 2 (1998) 39.

[14] OPAL Collaboration, K. Ahmet et al., Nucl. Instr. and Meth. A 305 (1991) 275.

[15] P.P. Allport et al., Nucl. Instr. and Meth. A 324 (1993) 34;

P.P. Allport et al., Nucl. Instr. and Meth. A 346 (1994) 476.

[16] M. Hauschild et al., Nucl. Instr. and Meth. A 314 (1992) 74.

[17] OPAL Collaboration, G. Alexander et al., Z. Phys. C 52 (1991) 175.

[18] B. Andersson et al., Phys. Rep. 97 (1983) 31;

T. Sjöstrand, Comp. Phys. Comm. 39 (1986) 347;

T. Sjöstrand and M. Bengtsson, Comp. Phys. Comm. 43 (1987) 367;

T. Sjöstrand, Comp. Phys. Comm. 82 (1994) 74.

[19] OPAL Collaboration, G. Alexander et al., Z. Phys. C 69 (1996) 543.

[20] OPAL Collaboration, M.Z. Akrawy et al., Z. Phys. C 47 (1990) 505.

[21] G. Marchesini and B.R. Webber, Nucl. Phys. B 310 (1988) 461;

G. Marchesini, B.R. Webber et al., Comp. Phys. Comm. 67 (1992) 465.

[22] J. Allison et al., Nucl. Instr. and Meth. A 317 (1992) 47. 
[23] M. Thomson, Nucl. Instr. and Meth. A 382 (1996) 553.

[24] OPAL Collaboration, K. Ackerstaff et al., Z. Phys. C 74 (1997) 1.

[25] F. James and M. Roos, "MINUIT-Function Minimization and Error Analysis", Version 95.03, CERN Program Library D506, CERN, Geneva 1995.

[26] The Particle Data Group, M. Aguilar-Benitez et al., Phys. Rev. D 54 (1996) 1.

[27] ALEPH collaboration, R. Barate et al., Z. Phys. C 74 (1997) 451;

OPAL Collaboration, K. Ackerstaff et al., Z. Phys. C 74 (1997) 413.

[28] L3 Collaboration, O. Adriani et al., Phys. Lett. B 292 (1992) 472.

[29] OPAL Collaboration, P.D. Acton et al, Z. Phys. C 56 (1992) 521.

[30] G.D. Lafferty, Z. Phys. C 60 (1993) 659.

[31] D.V. Bugg et al., Phys. Rev. D 50 (1994) 4412.

[32] Crystal Ball Collaboration, C. Amsler et al., Phys. Lett. B 327 (1994) 425.

WA76 Collaboration, T.A. Amstrong et al., Z. Phys. C 52 (1991) 389.

[33] E852 Collaboration, S. Teige et al., Meeting of Division of Particles and Field of the APS, August 1996, Minneapolis, USA. hep-ex/9608017.

[34] M.L. Stevenson et al., Phys. Rev. 125 (1962) 687;

D.W. Carpenter et al., Phys. Rev. D 1 (1970) 1303;

J.G. Layter et al., Phys. Rev. D 7 (1973) 2565.

[35] D. Alde et al., Phys. Lett. B 177 (1986) 115;

G.R. Kalbfleisch, Phys. Rev. D 10 (1974) 916.

[36] Y.L. Dokshitzer, V.A. Khoze and S.I. Troyan, J. Phys. G: Nucl. Part. Phys. 17 (1991) 1481

Y.L. Dokshitzer, V.A. Khoze and S.I. Troyan, Z. Phys. C 55 (1992) 107. 


\begin{tabular}{|l|c|c||c|}
\hline \hline Photon sample & $\gamma$ & $\gamma_{c}$ & $\gamma+\gamma_{c}$ \\
\hline$x_{E}$ range & $0.003-1.000$ & $0.003-1.000$ & $0.003-1.000$ \\
\hline Integrated rate & 16.79 & 16.96 & 16.84 \\
\hline \hline Errors (\%) & \multicolumn{3}{|l|}{} \\
\hline Statistics (data) & 0.1 & 0.2 & 0.1 \\
Statistics (Monte Carlo) & 0.1 & 0.1 & 0.1 \\
Difference between Monte Carlos & 3.4 & 3.0 & 3.3 \\
$P_{\gamma}(S)$ variations & 0.8 & - & 0.6 \\
Background subtraction & 0.8 & 0.5 & 0.7 \\
Nuclear interactions & 2.0 & 0.9 & 1.7 \\
Energy scale & 1.5 & 1.3 & 1.4 \\
Track and cal. simulation & 1.8 & 7.6 & 3.1 \\
\hline Total error (\%) & 4.7 & 8.3 & 5.1 \\
\hline \hline
\end{tabular}

Table 1: Number of photons per hadronic $\mathrm{Z}^{0}$ decay in the $x_{E}$ range covered by the measurement together with its statistical and systematic uncertainties (in \%). The three columns are the results obtained with the calorimetric sample $(\gamma)$, the conversion sample $\left(\gamma_{c}\right)$ and the combined sample $\left(\gamma+\gamma_{c}\right)$. 


\begin{tabular}{|c|c|c|c|c|}
\hline$\overline{\pi^{0}}$ sample & $\gamma \gamma$ & $\gamma \gamma_{c}$ & $\gamma_{c} \gamma_{c}$ & $\gamma \gamma+\gamma \gamma_{c}+\gamma_{c} \gamma_{c}$ \\
\hline$x_{E}$ Range & $0.007-0.500$ & $0.007-0.500$ & $0.009-0.300$ & $0.007-0.500$ \\
\hline Integrated rate & 8.37 & 8.12 & 7.51 & 8.29 \\
\hline Rate $\left(0.009<x_{E}<0.3\right)$ & 7.80 & 7.35 & 7.51 & 7.65 \\
\hline \multicolumn{5}{|l|}{ Errors (\%) } \\
\hline Statistics (data) & 0.6 & 2.1 & 4.9 & 0.6 \\
\hline Statistics (Monte Carlo) & 0.4 & 1.0 & 2.8 & 0.4 \\
\hline Difference between Monte Carlos & 4.7 & 4.0 & 5.9 & 4.1 \\
\hline$\tilde{P}_{\gamma \gamma}$ variations & 2.0 & 2.7 & 9.3 & 2.2 \\
\hline Background subtraction & 3.7 & 7.2 & 11.5 & 3.8 \\
\hline Gaussian peaks & 0.8 & 1.1 & 3.0 & 0.8 \\
\hline Nuclear interactions & 1.6 & 0.7 & 0.1 & 1.3 \\
\hline Energy scale (1\%) & 1.8 & 1.9 & 1.5 & 1.6 \\
\hline Background normalisation range & 4.3 & 2.3 & 5.0 & 3.1 \\
\hline Track and cal. simulation & 2.2 & 7.6 & 15.2 & 2.5 \\
\hline Total error $(\%)$ & 8.4 & 12.2 & 23.6 & 7.6 \\
\hline
\end{tabular}

Table 2: $\quad$ Statistical and systematic uncertainties (in \%) on the number of $\pi^{0}$ mesons per hadronic $\mathrm{Z}^{0}$ decay measured using the individual $\pi^{0} \rightarrow \gamma \gamma, \pi^{0} \rightarrow \gamma \gamma_{c}$ and $\pi^{0} \rightarrow \gamma_{c} \gamma_{c}$ samples, and the combined sample.

\begin{tabular}{|l|c|c||c|}
\hline \hline$\eta$ sample & $\gamma \gamma$ & $\gamma \gamma_{c}$ & $\gamma \gamma+\gamma \gamma_{c}$ \\
\hline \hline$x_{E}$ Range & $0.025-1.000$ & $0.040-0.300$ & $0.025-1.000$ \\
\hline Integrated rate & 0.746 & 0.569 & 0.745 \\
\hline Rate $\left(0.04<x_{E}<0.30\right)$ & 0.558 & 0.569 & 0.558 \\
\hline \hline Errors (\%) & \multicolumn{3}{|c|}{} \\
\hline Statistics (data) & 2.6 & 6.5 & 2.6 \\
Statistics (Monte Carlo) & 1.7 & 4.5 & 1.7 \\
Difference between Monte Carlos & 3.9 & 9.7 & 3.8 \\
$\tilde{P}_{\gamma \gamma}$ variations & 10.5 & 26.0 & 10.5 \\
Background subtraction & 7.4 & 16.7 & 7.3 \\
Gaussian peaks & 0.2 & 1.1 & 0.2 \\
Energy scale (1\%) & 0.8 & 0.4 & 0.8 \\
Background normalisation range & 1.9 & 3.9 & 1.9 \\
Track and cal. simulation & 1.1 & 6.9 & 1.1 \\
\hline Total Error (\%) & 14.0 & 34.3 & 13.9 \\
\hline \hline
\end{tabular}

Table 3: $\quad$ Statistical and systematic uncertainties (in \%) on the number of $\eta$ mesons per hadronic $\mathrm{Z}^{0}$ decay measured using the individual $\eta \rightarrow \gamma \gamma$ and $\eta \rightarrow \gamma \gamma_{c}$ samples, and the combined sample. 


\begin{tabular}{|l|c|c|c|c|c|}
\hline Meson & $\begin{array}{c}\eta \\
\pi^{0} \pi^{+} \pi^{-}\end{array}$ & $\begin{array}{c}\rho^{ \pm} \\
\pi^{0} \pi^{ \pm}\end{array}$ & $\begin{array}{c}\omega \\
\pi^{0} \pi^{+} \pi^{-}\end{array}$ & $\begin{array}{c}\eta^{\prime} \\
\eta \pi^{+} \pi^{-}\end{array}$ & $\begin{array}{c}\mathrm{a}_{0}^{ \pm} \\
\eta \pi^{ \pm}\end{array}$ \\
\hline$x_{E}$ range & $0.025-0.4$ & & $0.025-0.6$ & $0.05-0.8$ & \\
\hline $\log \left(1 / x_{p}\right)$ range & & $0.0-5.0$ & & & $0.0-3.5$ \\
\hline Rate & 0.898 & 2.36 & 0.883 & 0.103 & 0.214 \\
\hline \hline Errors (\%) & \multicolumn{5}{|l|}{} \\
\hline Statistics (data) & 6.5 & 2.3 & 3.6 & 10.1 & 16.8 \\
Statistics (Monte Carlo) & 1.7 & 1.7 & 0.5 & 1.6 & 2.5 \\
Difference between Monte Carlos & 3.7 & - & 5.9 & 4.9 & - \\
$\tilde{G}_{\gamma \gamma}(m)$ variations & 2.4 & 10.9 & 3.9 & 7.2 & 13.6 \\
Background subtraction & 4.5 & 7.4 & 3.0 & 5.0 & 30.4 \\
Mass resolution & 4.0 & 7.0 & 8.1 & 6.8 & - \\
Breit-Wigner extrapolation & - & 3.1 & - & - & - \\
Track and cal. simulation & 4.0 & 4.0 & 4.0 & 3.0 & 3.0 \\
Partial reconstruction & 6.6 & 9.0 & 5.8 & - & 6.6 \\
Branching Ratio & 2.5 & - & - & 3.4 & 10.3 \\
\hline Total Error (\%) & 12.9 & 18.4 & 13.7 & 16.5 & 39.4 \\
\hline \hline
\end{tabular}

Table 4: Statistical and systematic uncertainties (in \%) on the number of $\eta, \rho^{ \pm}, \omega, \eta^{\prime}$ and $\mathrm{a}_{0}^{ \pm}$ mesons per hadronic $\mathrm{Z}^{0}$ decay measured with the channels combining a $\pi^{0}$ or an $\eta$ meson with charged pions. For the $\rho^{ \pm}$and $\mathrm{a}_{0}^{ \pm}$resonances, the error associated with the difference between the Monte Carlos is included in the error associated with the background subtraction. 


\begin{tabular}{|c|c||c|c|}
\hline$x_{E}$ & $\frac{1}{\sigma_{\text {had }}} \frac{d \sigma}{d x_{E}}$ & $\ln \left(1 / x_{p}\right)$ & $\frac{1}{\sigma_{\text {had }}} \frac{d \sigma}{d \ln \left(1 / x_{p}\right)}$ \\
\hline \hline $0.003-0.004$ & $1309 \pm 13 \pm 330$ & $5.81-5.52$ & $4.55 \pm 0.05 \pm 1.15$ \\
$0.004-0.007$ & $986 \pm 2 \pm 78$ & $5.52-4.96$ & $5.29 \pm 0.01 \pm 0.42$ \\
$0.007-0.009$ & $749 \pm 1 \pm 34$ & $4.96-4.71$ & $5.96 \pm 0.01 \pm 0.27$ \\
$0.009-0.011$ & $613 \pm 1 \pm 28$ & $4.71-4.51$ & $6.11 \pm 0.01 \pm 0.28$ \\
$0.011-0.013$ & $508 \pm 1 \pm 23$ & $4.51-4.34$ & $6.08 \pm 0.02 \pm 0.27$ \\
$0.013-0.016$ & $404 \pm 1 \pm 16$ & $4.34-4.14$ & $5.84 \pm 0.01 \pm 0.23$ \\
$0.016-0.020$ & $303 \pm 1 \pm 11$ & $4.14-3.91$ & $5.43 \pm 0.01 \pm 0.20$ \\
$0.020-0.025$ & $225 \pm 1 \pm 9$ & $3.91-3.69$ & $5.03 \pm 0.01 \pm 0.19$ \\
$0.025-0.030$ & $167 \pm 1 \pm 7$ & $3.69-3.51$ & $4.58 \pm 0.01 \pm 0.18$ \\
$0.030-0.035$ & $131 \pm 1 \pm 5$ & $3.51-3.35$ & $4.24 \pm 0.01 \pm 0.15$ \\
$0.035-0.040$ & $103 \pm 1 \pm 4$ & $3.35-3.22$ & $3.87 \pm 0.01 \pm 0.15$ \\
$0.040-0.050$ & $76.9 \pm 0.2 \pm 2.8$ & $3.22-3.00$ & $3.45 \pm 0.01 \pm 0.13$ \\
$0.050-0.060$ & $53.4 \pm 0.2 \pm 2.0$ & $3.00-2.81$ & $2.93 \pm 0.01 \pm 0.11$ \\
$0.060-0.070$ & $39.2 \pm 0.2 \pm 1.4$ & $2.81-2.66$ & $2.54 \pm 0.01 \pm 0.09$ \\
$0.070-0.085$ & $28.1 \pm 0.1 \pm 1.0$ & $2.66-2.47$ & $2.17 \pm 0.01 \pm 0.08$ \\
$0.085-0.100$ & $19.6 \pm 0.1 \pm 0.8$ & $2.47-2.30$ & $1.81 \pm 0.01 \pm 0.08$ \\
$0.100-0.125$ & $13.0 \pm 0.1 \pm 0.5$ & $2.30-2.08$ & $1.45 \pm 0.01 \pm 0.06$ \\
$0.125-0.150$ & $8.04 \pm 0.05 \pm 0.35$ & $2.08-1.90$ & $1.103 \pm 0.006 \pm 0.048$ \\
$0.150-0.200$ & $4.50 \pm 0.03 \pm 0.26$ & $1.90-1.61$ & $0.783 \pm 0.005 \pm 0.045$ \\
$0.200-0.300$ & $1.71 \pm 0.02 \pm 0.19$ & $1.61-1.20$ & $0.422 \pm 0.004 \pm 0.047$ \\
$0.300-0.400$ & $0.507 \pm 0.010 \pm 0.087$ & $1.20-0.92$ & $0.176 \pm 0.003 \pm 0.030$ \\
$0.400-0.500$ & $0.184 \pm 0.005 \pm 0.038$ & $0.92-0.69$ & $0.082 \pm 0.002 \pm 0.017$ \\
$0.500-0.600$ & $0.065 \pm 0.002 \pm 0.011$ & $0.69-0.51$ & $0.036 \pm 0.001 \pm 0.006$ \\
$0.600-0.800$ & $0.017 \pm 0.001 \pm 0.002$ & $0.51-0.22$ & $0.012 \pm 0.000 \pm 0.002$ \\
$0.800-1.000$ & $0.0023 \pm 0.0003 \pm 0.0010$ & $0.22-0.00$ & $0.0020 \pm 0.0003 \pm 0.0009$ \\
\hline \hline
\end{tabular}

Table 5: Photon fragmentation function obtained by combining the calorimeter and conversion results. The quoted errors are statistical and systematic, respectively. 


\begin{tabular}{|c|c||c|c|}
\hline$x_{E}$ & $\frac{1}{\sigma_{\text {had }}} \frac{d \sigma}{d x_{E}}$ & $\ln \left(1 / x_{p}\right)$ & $\frac{1}{\sigma_{\text {had }}} \frac{d \sigma}{d \ln \left(1 / x_{p}\right)}$ \\
\hline \hline $0.007-0.009$ & $254 \pm 18 \pm 48$ & $5.06-4.77$ & $1.74 \pm 0.12 \pm 0.33$ \\
$0.009-0.011$ & $266 \pm 12 \pm 38$ & $4.77-4.55$ & $2.42 \pm 0.11 \pm 0.34$ \\
$0.011-0.013$ & $248 \pm 6 \pm 28$ & $4.55-4.37$ & $2.78 \pm 0.06 \pm 0.31$ \\
$0.013-0.016$ & $211 \pm 3 \pm 18$ & $4.37-4.15$ & $2.92 \pm 0.05 \pm 0.25$ \\
$0.016-0.020$ & $178 \pm 2 \pm 14$ & $4.15-3.92$ & $3.11 \pm 0.03 \pm 0.25$ \\
$0.020-0.025$ & $139 \pm 2 \pm 6$ & $3.92-3.70$ & $3.05 \pm 0.03 \pm 0.13$ \\
$0.025-0.030$ & $113 \pm 1 \pm 5$ & $3.70-3.51$ & $3.06 \pm 0.03 \pm 0.13$ \\
$0.030-0.035$ & $94.1 \pm 0.9 \pm 4.0$ & $3.51-3.36$ & $3.03 \pm 0.03 \pm 0.13$ \\
$0.035-0.040$ & $77.7 \pm 0.8 \pm 4.3$ & $3.36-3.22$ & $2.89 \pm 0.03 \pm 0.16$ \\
$0.040-0.050$ & $62.5 \pm 0.4 \pm 3.9$ & $3.22-3.00$ & $2.79 \pm 0.02 \pm 0.17$ \\
$0.050-0.060$ & $45.7 \pm 0.4 \pm 3.0$ & $3.00-2.81$ & $2.50 \pm 0.02 \pm 0.16$ \\
$0.060-0.070$ & $34.7 \pm 0.3 \pm 3.0$ & $2.81-2.66$ & $2.25 \pm 0.02 \pm 0.20$ \\
$0.070-0.085$ & $26.2 \pm 0.2 \pm 1.8$ & $2.66-2.47$ & $2.02 \pm 0.02 \pm 0.14$ \\
$0.085-0.100$ & $19.4 \pm 0.2 \pm 1.4$ & $2.47-2.30$ & $1.79 \pm 0.02 \pm 0.13$ \\
$0.100-0.125$ & $13.2 \pm 0.1 \pm 2.9$ & $2.30-2.08$ & $1.48 \pm 0.02 \pm 0.32$ \\
$0.125-0.150$ & $9.05 \pm 0.13 \pm 0.76$ & $2.08-1.90$ & $1.240 \pm 0.017 \pm 0.105$ \\
$0.150-0.200$ & $5.36 \pm 0.10 \pm 0.69$ & $1.90-1.61$ & $0.931 \pm 0.017 \pm 0.120$ \\
$0.200-0.300$ & $2.26 \pm 0.13 \pm 0.38$ & $1.61-1.20$ & $0.558 \pm 0.031 \pm 0.094$ \\
$0.300-0.400$ & $0.764 \pm 0.085 \pm 0.309$ & $1.20-0.92$ & $0.266 \pm 0.030 \pm 0.107$ \\
$0.400-0.500$ & $0.455 \pm 0.095 \pm 0.244$ & $0.92-0.69$ & $0.204 \pm 0.043 \pm 0.110$ \\
\hline \hline
\end{tabular}

Table 6: $\quad \pi^{0}$ fragmentation function obtained by combining the $\pi^{0} \rightarrow \gamma \gamma, \pi^{0} \rightarrow \gamma \gamma_{c}$ and $\pi^{0} \rightarrow \gamma_{c} \gamma_{c}$ data. The quoted errors are statistical and systematic, respectively.

\begin{tabular}{|c|c||c|c|}
\hline$x_{E}$ & $\frac{1}{\sigma_{\text {had }}} \frac{d \sigma}{d x_{p}}$ & $\ln \left(1 / x_{p}\right)$ & $\frac{1}{\sigma_{\text {had }}} \frac{d \sigma}{d \ln \left(1 / x_{p}\right)}$ \\
\hline \hline $0.025-0.035$ & $10.6 \pm 1.5 \pm 2.4$ & $3.82-3.42$ & $0.261 \pm 0.038 \pm 0.046$ \\
$0.035-0.050$ & $7.63 \pm 0.78 \pm 1.27$ & $3.42-3.03$ & $0.294 \pm 0.030 \pm 0.038$ \\
$0.050-0.075$ & $5.10 \pm 0.38 \pm 0.61$ & $3.03-2.60$ & $0.302 \pm 0.023 \pm 0.028$ \\
$0.075-0.100$ & $3.81 \pm 0.21 \pm 0.44$ & $2.60-2.31$ & $0.324 \pm 0.018 \pm 0.032$ \\
$0.100-0.125$ & $2.83 \pm 0.12 \pm 0.28$ & $2.31-2.08$ & $0.314 \pm 0.014 \pm 0.028$ \\
$0.125-0.150$ & $2.21 \pm 0.10 \pm 0.22$ & $2.08-1.90$ & $0.301 \pm 0.014 \pm 0.027$ \\
$0.150-0.200$ & $1.46 \pm 0.05 \pm 0.13$ & $1.90-1.61$ & $0.252 \pm 0.009 \pm 0.021$ \\
$0.200-0.300$ & $0.733 \pm 0.026 \pm 0.062$ & $1.61-1.20$ & $0.180 \pm 0.006 \pm 0.014$ \\
$0.300-0.400$ & $0.364 \pm 0.022 \pm 0.047$ & $1.20-0.92$ & $0.126 \pm 0.008 \pm 0.014$ \\
$0.400-0.500$ & $0.220 \pm 0.019 \pm 0.031$ & $0.92-0.69$ & $0.099 \pm 0.008 \pm 0.011$ \\
$0.500-0.600$ & $0.086 \pm 0.010 \pm 0.019$ & $0.69-0.51$ & $0.047 \pm 0.006 \pm 0.009$ \\
$0.600-0.800$ & $0.033 \pm 0.004 \pm 0.008$ & $0.51-0.22$ & $0.023 \pm 0.003 \pm 0.005$ \\
$0.800-1.000$ & $0.0013 \pm 0.0004 \pm 0.0011$ & $0.22-0.00$ & $0.0012 \pm 0.0004 \pm 0.0009$ \\
\hline \hline
\end{tabular}

Table 7: $\quad \eta$ fragmentation function obtained by combining the $\gamma \gamma, \gamma \gamma_{c}$ and $\pi^{0} \pi^{+} \pi^{-}$data. The quoted errors are statistical and systematic, respectively. 


\begin{tabular}{|c|c||c|c|}
\hline$x_{E}$ & $\frac{1}{\sigma_{\text {had }}} \frac{d \sigma}{d x_{p}}$ & $\ln \left(1 / x_{p}\right)$ & $\frac{1}{\sigma_{\text {had }}} \frac{d \sigma}{d \ln \left(1 / x_{p}\right)}$ \\
\hline \hline $0.016-0.025$ & $17.3 \pm 8.1 \pm 12.2$ & $5.0-4.5$ & $0.171 \pm 0.008 \pm 0.081$ \\
$0.025-0.035$ & $32.3 \pm 2.5 \pm 9.7$ & $4.5-4.0$ & $0.419 \pm 0.035 \pm 0.111$ \\
$0.035-0.050$ & $21.3 \pm 0.7 \pm 4.5$ & $4.0-3.5$ & $0.500 \pm 0.092 \pm 0.138$ \\
$0.050-0.075$ & $16.7 \pm 0.4 \pm 1.8$ & $3.5-3.0$ & $0.692 \pm 0.028 \pm 0.165$ \\
$0.075-0.100$ & $9.89 \pm 0.40 \pm 1.46$ & $3.0-2.5$ & $0.868 \pm 0.021 \pm 0.126$ \\
$0.100-0.125$ & $7.11 \pm 0.25 \pm 1.04$ & $2.5-2.0$ & $0.805 \pm 0.022 \pm 0.104$ \\
$0.125-0.150$ & $5.90 \pm 0.25 \pm 0.78$ & $2.0-1.5$ & $0.603 \pm 0.017 \pm 0.078$ \\
$0.150-0.200$ & $3.60 \pm 0.12 \pm 0.48$ & $1.5-1.0$ & $0.419 \pm 0.014 \pm 0.073$ \\
$0.200-0.300$ & $2.02 \pm 0.07 \pm 0.21$ & $1.0-0.5$ & $0.217 \pm 0.010 \pm 0.055$ \\
$0.300-0.400$ & $1.03 \pm 0.04 \pm 0.27$ & $0.5-0.0$ & $0.034 \pm 0.004 \pm 0.019$ \\
$0.400-0.600$ & $0.430 \pm 0.023 \pm 0.081$ & & \\
$0.600-0.800$ & $0.075 \pm 0.013 \pm 0.032$ & & \\
$0.800-1.000$ & $0.013 \pm 0.003 \pm 0.009$ & & \\
\hline \hline
\end{tabular}

Table 8: $\quad \rho^{ \pm}$fragmentation function. The quoted errors are statistical and systematic, respectively. Because of the width of the $\rho^{ \pm}$, the relation between $x_{E}$ and $x_{p}$ varies with mass. Therefore the analysis is performed first with bins of $x_{E}$ (first two columns) and then repeated with bins of $\log \left(1 / x_{p}\right)$.

\begin{tabular}{|c|c||c|c|}
\hline$x_{E}$ & $\frac{1}{\sigma_{\text {had }}} \frac{d \sigma}{d x_{p}}$ & $\ln \left(1 / x_{p}\right)$ & $\frac{1}{\sigma_{\text {had }}} \frac{d \sigma}{d \ln \left(1 / x_{p}\right)}$ \\
\hline \hline $0.025-0.035$ & $15.2 \pm 2.4 \pm 2.1$ & $4.01-3.49$ & $0.293 \pm 0.046 \pm 0.040$ \\
$0.035-0.050$ & $9.88 \pm 0.84 \pm 1.48$ & $3.49-3.06$ & $0.344 \pm 0.029 \pm 0.051$ \\
$0.050-0.075$ & $5.82 \pm 0.35 \pm 0.75$ & $3.06-2.62$ & $0.330 \pm 0.020 \pm 0.043$ \\
$0.075-0.100$ & $4.12 \pm 0.25 \pm 0.54$ & $2.62-2.32$ & $0.344 \pm 0.021 \pm 0.045$ \\
$0.100-0.125$ & $2.74 \pm 0.16 \pm 0.32$ & $2.32-2.09$ & $0.299 \pm 0.018 \pm 0.035$ \\
$0.125-0.150$ & $2.23 \pm 0.14 \pm 0.24$ & $2.09-1.90$ & $0.301 \pm 0.018 \pm 0.032$ \\
$0.150-0.200$ & $1.45 \pm 0.09 \pm 0.17$ & $1.90-1.61$ & $0.250 \pm 0.016 \pm 0.029$ \\
$0.200-0.300$ & $0.789 \pm 0.049 \pm 0.099$ & $1.61-1.21$ & $0.193 \pm 0.012 \pm 0.024$ \\
$0.300-0.400$ & $0.335 \pm 0.037 \pm 0.042$ & $1.21-0.92$ & $0.116 \pm 0.013 \pm 0.014$ \\
$0.400-0.600$ & $0.130 \pm 0.027 \pm 0.028$ & $0.92-0.51$ & $0.064 \pm 0.013 \pm 0.014$ \\
\hline \hline
\end{tabular}

Table 9: $\omega$ fragmentation function. The quoted errors are statistical and systematic, respectively. 


\begin{tabular}{|c|c||c|c|}
\hline$x_{E}$ & $\frac{1}{\sigma_{\text {had }}} \frac{d \sigma}{d x_{p}}$ & $\ln \left(1 / x_{p}\right)$ & $\frac{1}{\sigma_{\text {had }}} \frac{d \sigma}{d \ln \left(1 / x_{p}\right)}$ \\
\hline \hline $0.050-0.070$ & $1.01 \pm 0.38 \pm 0.14$ & $3.09-2.71$ & $0.052 \pm 0.020 \pm 0.007$ \\
$0.070-0.100$ & $0.462 \pm 0.180 \pm 0.073$ & $2.71-2.33$ & $0.036 \pm 0.014 \pm 0.006$ \\
$0.100-0.125$ & $0.460 \pm 0.144 \pm 0.082$ & $2.33-2.09$ & $0.050 \pm 0.016 \pm 0.009$ \\
$0.125-0.150$ & $0.293 \pm 0.099 \pm 0.049$ & $2.09-1.91$ & $0.039 \pm 0.013 \pm 0.007$ \\
$0.150-0.200$ & $0.354 \pm 0.068 \pm 0.054$ & $1.91-1.61$ & $0.061 \pm 0.012 \pm 0.009$ \\
$0.200-0.300$ & $0.137 \pm 0.028 \pm 0.017$ & $1.61-1.21$ & $0.034 \pm 0.007 \pm 0.004$ \\
$0.300-0.400$ & $0.088 \pm 0.020 \pm 0.011$ & $1.21-0.92$ & $0.030 \pm 0.007 \pm 0.004$ \\
$0.400-0.600$ & $0.034 \pm 0.010 \pm 0.006$ & $0.92-0.51$ & $0.017 \pm 0.005 \pm 0.003$ \\
$0.600-0.800$ & $0.013 \pm 0.006 \pm 0.003$ & $0.51-0.22$ & $0.009 \pm 0.004 \pm 0.002$ \\
\hline \hline
\end{tabular}

Table 10: $\eta^{\prime}$ fragmentation function. The quoted errors are statistical and systematic, respectively.

\begin{tabular}{|c|c||c|c|}
\hline$x_{E}$ & $\frac{1}{\sigma_{\text {had }}} \frac{d \sigma}{d x_{p}}$ & $\ln \left(1 / x_{p}\right)$ & $\frac{1}{\sigma_{\text {had }}} \frac{d \sigma}{d \ln \left(1 / x_{p}\right)}$ \\
\hline \hline $0.050-0.070$ & $1.65 \pm 1.03 \pm 0.75$ & $3.50-3.00$ & $0.093 \pm 0.063 \pm 0.050$ \\
$0.070-0.100$ & $1.05 \pm 0.49 \pm 0.73$ & $3.00-2.50$ & $0.104 \pm 0.041 \pm 0.041$ \\
$0.100-0.125$ & $0.747 \pm 0.215 \pm 0.214$ & $2.50-2.00$ & $0.076 \pm 0.019 \pm 0.030$ \\
$0.125-0.150$ & $0.985 \pm 0.238 \pm 0.560$ & $2.00-1.50$ & $0.088 \pm 0.013 \pm 0.023$ \\
$0.150-0.200$ & $0.623 \pm 0.107 \pm 0.171$ & $1.50-1.00$ & $0.040 \pm 0.009 \pm 0.012$ \\
$0.200-0.300$ & $0.207 \pm 0.046 \pm 0.069$ & $1.00-0.50$ & $0.019 \pm 0.006 \pm 0.007$ \\
$0.300-0.400$ & $0.093 \pm 0.027 \pm 0.040$ & $0.50-0.00$ & $0.0071 \pm 0.0025 \pm 0.0022$ \\
$0.400-0.600$ & $0.038 \pm 0.015 \pm 0.015$ & & \\
$0.600-0.800$ & $0.014 \pm 0.005 \pm 0.006$ & & \\
$0.800-1.000$ & $0.0040 \pm 0.0018 \pm 0.0024$ & & \\
\hline \hline
\end{tabular}

Table 11: $\quad \mathrm{a}_{0}^{ \pm}$fragmentation function. The quoted errors are statistical and systematic, respectively. Because of the width of the $\mathrm{a}_{0}^{ \pm}$, the relation between $x_{E}$ and $x_{p}$ varies with mass. Therefore the analysis is performed first with bins of $x_{E}$ (first two columns) and then repeated with bins of $\log \left(1 / x_{p}\right)$. 


\begin{tabular}{|c|c|c|c|c|c|}
\hline \multirow{2}{*}{ Particle } & \multicolumn{5}{|c|}{ Location of the maximum of $d \sigma / d \ln \left(1 / x_{p}\right)$} \\
\cline { 2 - 6 } & OPAL & L3 [10]12] & DELPHI [9] & JETSET 7.4 & HERWIG 5.9 \\
\hline$\gamma$ & $4.61 \pm 0.12$ & & & 4.54 & 4.63 \\
$\pi^{0}$ & $3.77 \pm 0.11$ & $3.90 \pm_{0.14}^{0.24}$ & $3.96 \pm 0.13$ & 3.78 & 3.86 \\
$\eta$ & $2.64 \pm 0.14$ & $2.52 \pm 0.12$ & & 2.94 & 3.01 \\
$\rho^{ \pm}$ & $2.63 \pm 0.15$ & & & 2.69 & 2.70 \\
$\omega$ & $2.89 \pm 0.24$ & $2.86 \pm 0.20^{*}$ & & 2.77 & 2.77 \\
$\eta^{\prime}$ & $2.21 \pm 0.42$ & $2.69 \pm 0.10^{*}$ & & 2.48 & 2.17 \\
$\mathrm{a}_{0}^{ \pm}$ & $2.57 \pm 0.50$ & & & 2.62 & 2.72 \\
\hline \hline
\end{tabular}

Table 12: Location of the maximum of the $\log \left(1 / x_{p}\right)$ distributions, determined from a Gaussian fit to the data in the region around the maximum. The OPAL measurements are compared to other LEP measurements and to the predictions of the default versions of JETSET 7.4 and HERWIG 5.9. The errors on the predictions are typically \pm 0.01 . The results marked with an asterisk are extracted assuming that the shape of the $\log \left(1 / x_{p}\right)$ distribution is given by a MLLA calculation [12].

\begin{tabular}{|l|r|r|r|r|r|r|r|}
\hline & $\gamma$ & $\pi^{0}$ & $\eta$ & $\rho^{ \pm}$ & $\omega$ & $\eta^{\prime}$ & $\mathrm{a}_{0}^{ \pm}$ \\
\hline \hline Measured range: & & & & & & & \\
Min. $\log \left(1 / x_{p}\right)$ & 0.00 & 0.69 & 0.00 & 0.00 & 0.51 & 0.22 & 0.00 \\
Max. $\log \left(1 / x_{p}\right)$ & 5.81 & 5.06 & 3.82 & 5.00 & 4.01 & 3.09 & 3.50 \\
\hline \% of rate in range & & & & & & & \\
JETSET 7.4 & 81.9 & 88.7 & 78.7 & 98.9 & 87.7 & 68.9 & $80.5^{*}$ \\
HERWIG 5.9 & 79.4 & 87.7 & 77.9 & 98.7 & 87.1 & 74.5 & 78.6 \\
Gaussian fit & 78.7 & 84.9 & 82.2 & 97.4 & 82.3 & 77.7 & 81.6 \\
$\pi^{ \pm}$shape & & 88.4 & & & & & \\
$\mathrm{~K}_{\mathrm{S}}^{0}$ shape & & & 82.2 & & & & \\
\hline Combined \% & 80.3 & 86.8 & 81.1 & 98.2 & 85.0 & 73.3 & 80.1 \\
Error & 1.6 & 1.9 & 3.1 & 0.8 & 2.7 & 4.4 & 1.5 \\
\hline Measured rate & 16.84 & 8.29 & 0.789 & 2.36 & 0.883 & 0.103 & 0.214 \\
Extrapolated rate & 20.97 & 9.55 & 0.973 & 2.40 & 1.039 & 0.141 & 0.267 \\
Extrapolation error & 0.42 & 0.21 & 0.038 & 0.02 & 0.033 & 0.008 & 0.005 \\
\hline \hline
\end{tabular}

Table 13: Data used for the extrapolation to the unobserved energy/momentum ranges. The result marked with an asterisk is from the JETSET tune of ref. [19]. 


\begin{tabular}{|c|c|c|c|c|c|c|}
\hline & \multicolumn{4}{|c|}{ Experimental results } & \multirow{2}{*}{$\begin{array}{c}\text { JETSET } \\
7.4 \\
\end{array}$} & \multirow{2}{*}{$\begin{array}{c}\text { HERWIG } \\
5.9 \\
\end{array}$} \\
\hline & OPAL & ALEPH [6] & DELPHI [9] & L3 [10 12 & & \\
\hline $\begin{array}{c}\text { photon } \\
x_{E} \text { range } \\
N_{\gamma} \text { in range } \\
N_{\gamma} \text { all } x_{E} \\
\end{array}$ & $\begin{array}{c}0.003-1.000 \\
16.84 \pm 0.86 \\
20.97 \pm 1.15 \\
\end{array}$ & $\begin{array}{l}0.018-0.450 \\
7.37 \pm 0.24\end{array}$ & & & 20.76 & 22.65 \\
\hline $\begin{array}{c}\pi^{0} \\
x_{E} \text { range } \\
N_{\pi^{0}} \text { in range } \\
N_{\pi^{0}} \text { all } x_{E} \\
\end{array}$ & $\begin{array}{l}0.007-0.400 \\
8.29 \pm 0.63 \\
9.55 \pm 0.76\end{array}$ & $\begin{array}{l}0.025-1.000 \\
4.80 \pm 0.32 \\
9.63 \pm 0.64\end{array}$ & $\begin{array}{c}0.011-0.750 \\
7.1 \pm 0.8 \\
9.2 \pm 1.0\end{array}$ & $\begin{array}{l}0.004-0.150 \\
8.38 \pm 0.67 \\
9.18 \pm 0.73\end{array}$ & 9.60 & 10.29 \\
\hline $\begin{array}{c}\eta \\
x_{E} \text { range } \\
N_{\eta} \text { in range } \\
N_{\eta} \text { all } x_{E} \\
N_{\eta} x_{p}>0.1 \\
\end{array}$ & $\begin{array}{c}0.025-1.000 \\
0.79 \pm 0.08 \\
0.97 \pm 0.11 \\
0.344 \pm 0.030 \\
\end{array}$ & $\begin{array}{c}0.100-1.000 \\
0.282 \pm 0.022 \\
0.282 \pm 0.022 \\
\end{array}$ & & $\begin{array}{l}0.020-0.300 \\
0.70 \pm 0.08 \\
0.91 \pm 0.11\end{array}$ & $\begin{array}{c}1.00 \\
0.286 \\
\end{array}$ & $\begin{array}{c}0.92 \\
0.243 \\
\end{array}$ \\
\hline $\begin{array}{c}\rho^{ \pm} \\
x_{E} \text { range } \\
N_{\rho^{ \pm}} \text {in range } \\
N_{\rho^{ \pm}} \text {all } x_{E} \\
\end{array}$ & $\begin{array}{l}0.016-1.000 \\
2.36 \pm 0.42 \\
2.40 \pm 0.44\end{array}$ & & & & 2.82 & 2.29 \\
\hline $\begin{array}{c}\omega \\
x_{E} \text { range } \\
N_{\omega} \text { in range } \\
N_{\omega} \text { all } x_{E} \\
\end{array}$ & $\begin{array}{l}0.025-0.800 \\
0.88 \pm 0.12 \\
1.04 \pm 0.14 \\
\end{array}$ & $\begin{array}{l}0.053-1.000 \\
0.64 \pm 0.08 \\
1.07 \pm 0.14\end{array}$ & & $\begin{array}{l}0.026-0.300 \\
0.94 \pm 0.14 \\
1.17 \pm 0.17\end{array}$ & 1.35 & 1.14 \\
\hline $\begin{array}{c}\eta^{\prime} \\
x_{E} \text { range } \\
N_{\eta^{\prime}} \text { in range } \\
N_{\eta^{\prime}} \text { all } x_{E} \\
N_{\eta}^{\prime} x_{p}>0.1 \\
\end{array}$ & $\begin{array}{c}0.050-0.800 \\
0.103 \pm 0.017 \\
0.14 \pm 0.03 \\
0.069 \pm 0.012\end{array}$ & $\begin{array}{c}0.100-1.000 \\
0.064 \pm 0.014 \\
0.064 \pm 0.014\end{array}$ & & $\begin{array}{l}0.023-0.240 \\
0.25 \pm 0.04\end{array}$ & $\begin{array}{l}0.297 \\
0.127 \\
\end{array}$ & $\begin{array}{l}0.122 \\
0.060 \\
\end{array}$ \\
\hline $\begin{array}{c}\mathrm{a}_{0}^{ \pm} \\
x_{E} \text { range } \\
N_{\mathrm{a}_{0}^{ \pm}} \text {in range } \\
N_{\mathrm{a}_{0}^{ \pm}} \text {all } x_{E}\end{array}$ & $\begin{array}{c}0.050-1.000 \\
0.21 \pm 0.08 \\
0.27 \pm 0.11\end{array}$ & & & & 0.210 & 0.221 \\
\hline
\end{tabular}

Table 14: Summary of the measurements of the particles rates described in this paper compared to other measurements at LEP and to JETSET and HERWIG predictions. 
OPAL

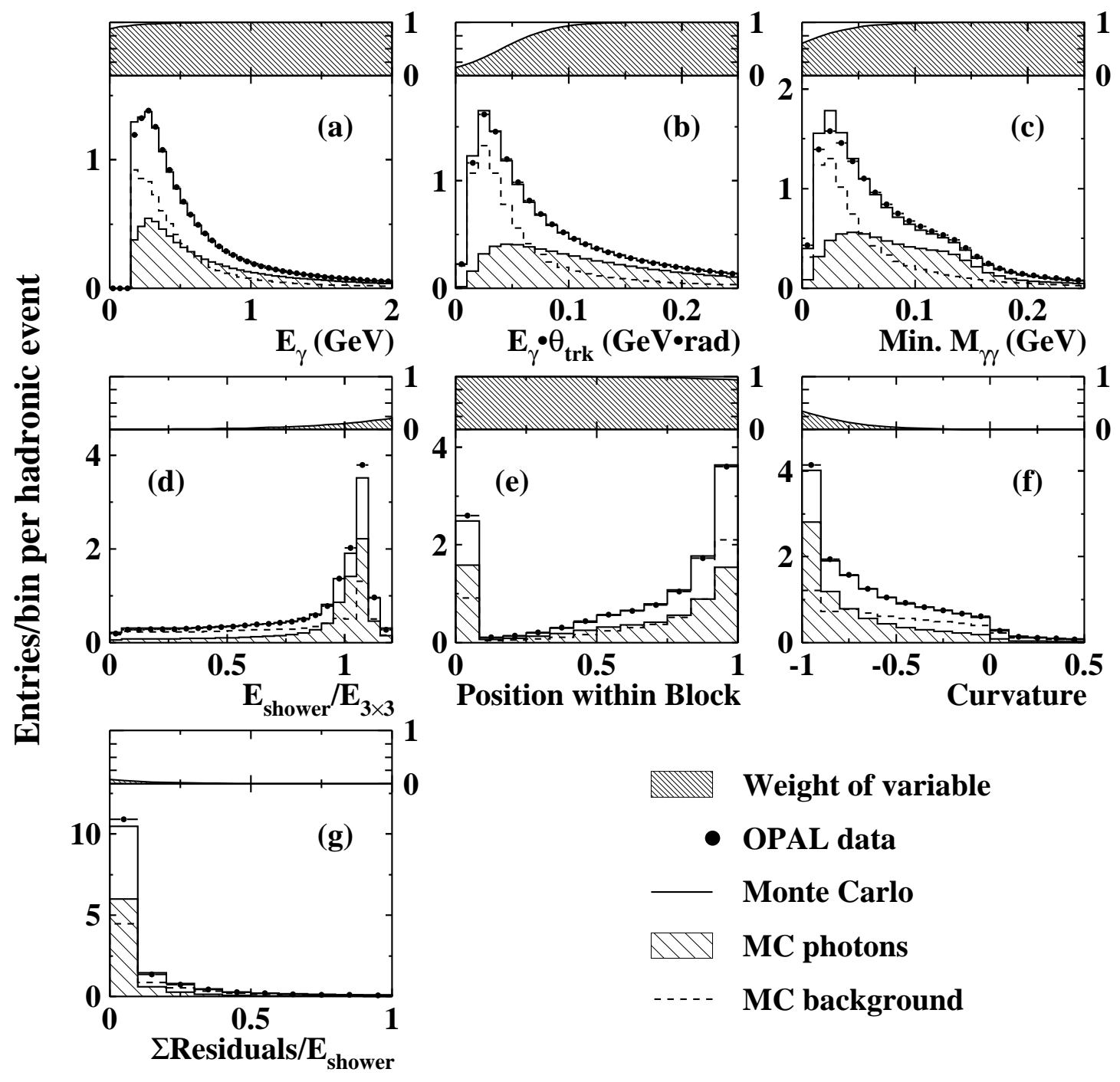

Figure 1: Distribution of variables used for the discrimination of photons recorded in the electromagnetic calorimeter. The points represent the data and the histogram represents the Monte Carlo simulation, normalised to one event. The Monte Carlo photons and background are shown as hatched and dashed histograms, respectively. The insert shows the weights used in eq. 1. The variables are: a) the photon energy; b) the photon energy multiplied by the angle to the closest charged track; c) the minimum of the invariant mass of the photon with any other photon; d) the fitted shower energy divided by the sum of the energy in the $3 \times 3$ array of blocks around the shower; e) the distance of the shower relative to the centre of the block, a value of 1 corresponding to the edge of the block; f) the maximum value of $\left(E_{-1}-E_{0}\right)\left(E_{+1}-E_{0}\right) / E_{0}^{2}$, where $E_{-1}, E_{0}, E_{+1}$ are the energies deposited in 3 consecutive blocks in either $\theta$ or $\phi$, the index 0 corresponding to the block where the shower is centered; and $g$ ) the sum of the residual of the shower fit in the $3 \times 3$ array of blocks around the shower divided by the shower energy. 


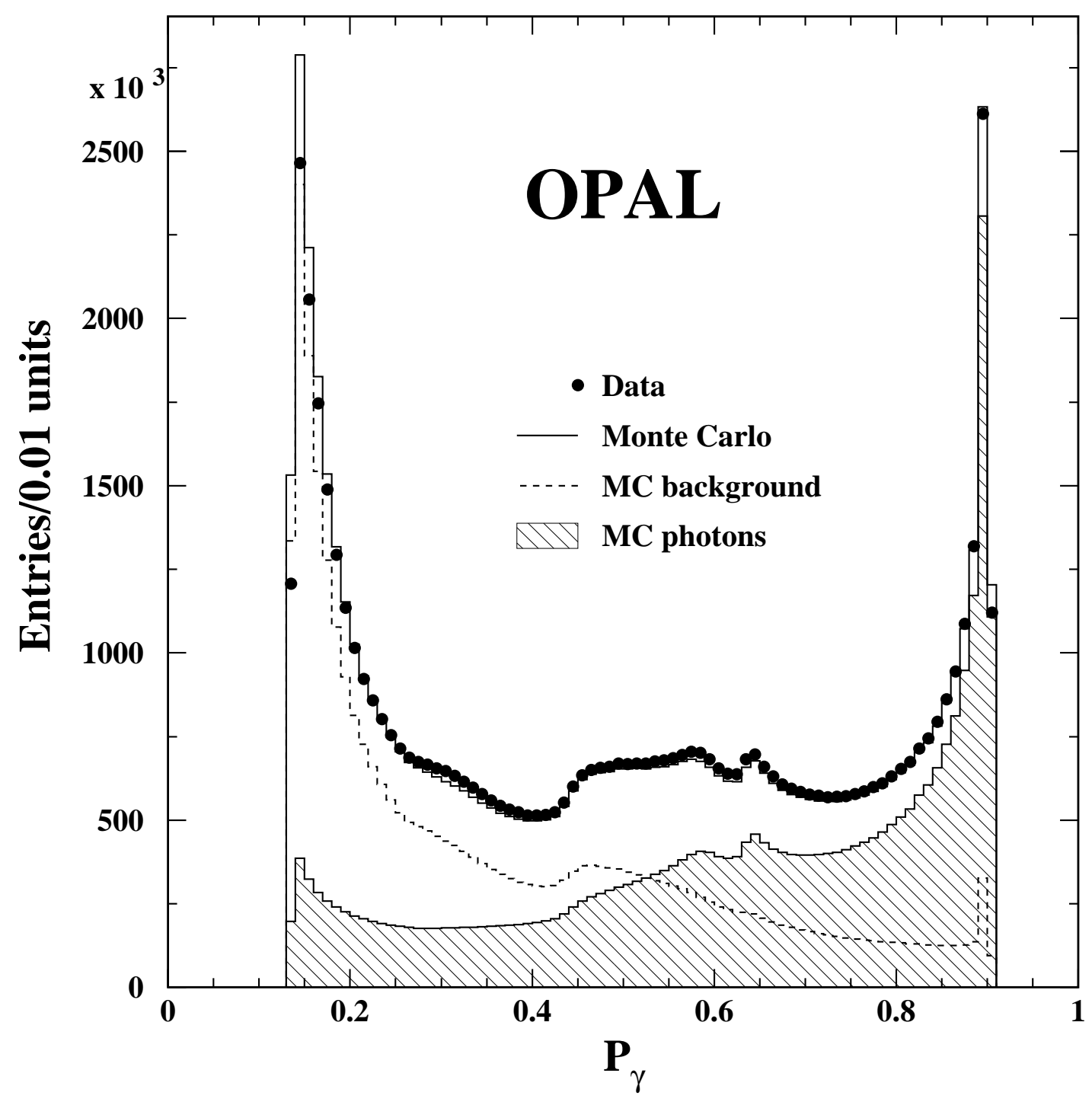

Figure 2: Distribution of $P_{\gamma}$, the variable used for the selection of calorimeter photons in the measurement of the photon rates. The points are data and the histogram represents the Monte Carlo simulation. The Monte Carlo signal (dashed histogram) and background (hatched histogram) are also shown. The Monte Carlo signal has been normalised to the measured photon rate and the background to the number of events in the data sample. 


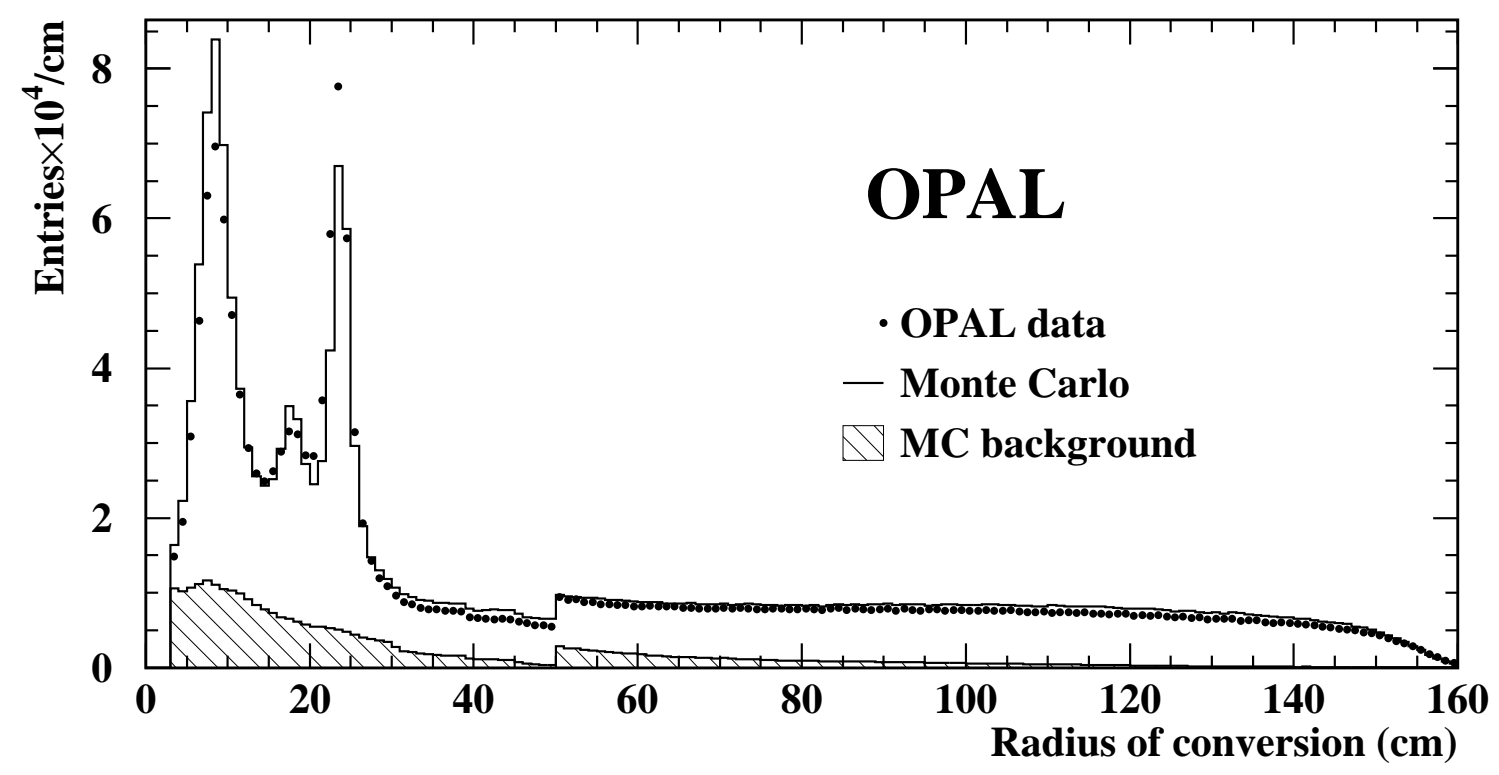

Figure 3: Radial coordinate $r$ of the photon conversion, in the data (dots) and in the Monte Carlo (histogram). The shaded histogram shows the contribution from background, according to the Monte Carlo. The Monte Carlo sample is normalised to the same number of events. The peaks close to 9, 18 and $23 \mathrm{~cm}$ correspond to concentration of material in the detector. The abrupt cut at $r=50 \mathrm{~cm}$ is due to an additional cut on the $\mathrm{d} E / \mathrm{d} x$ of the two electron tracks. 


\section{OPAL}
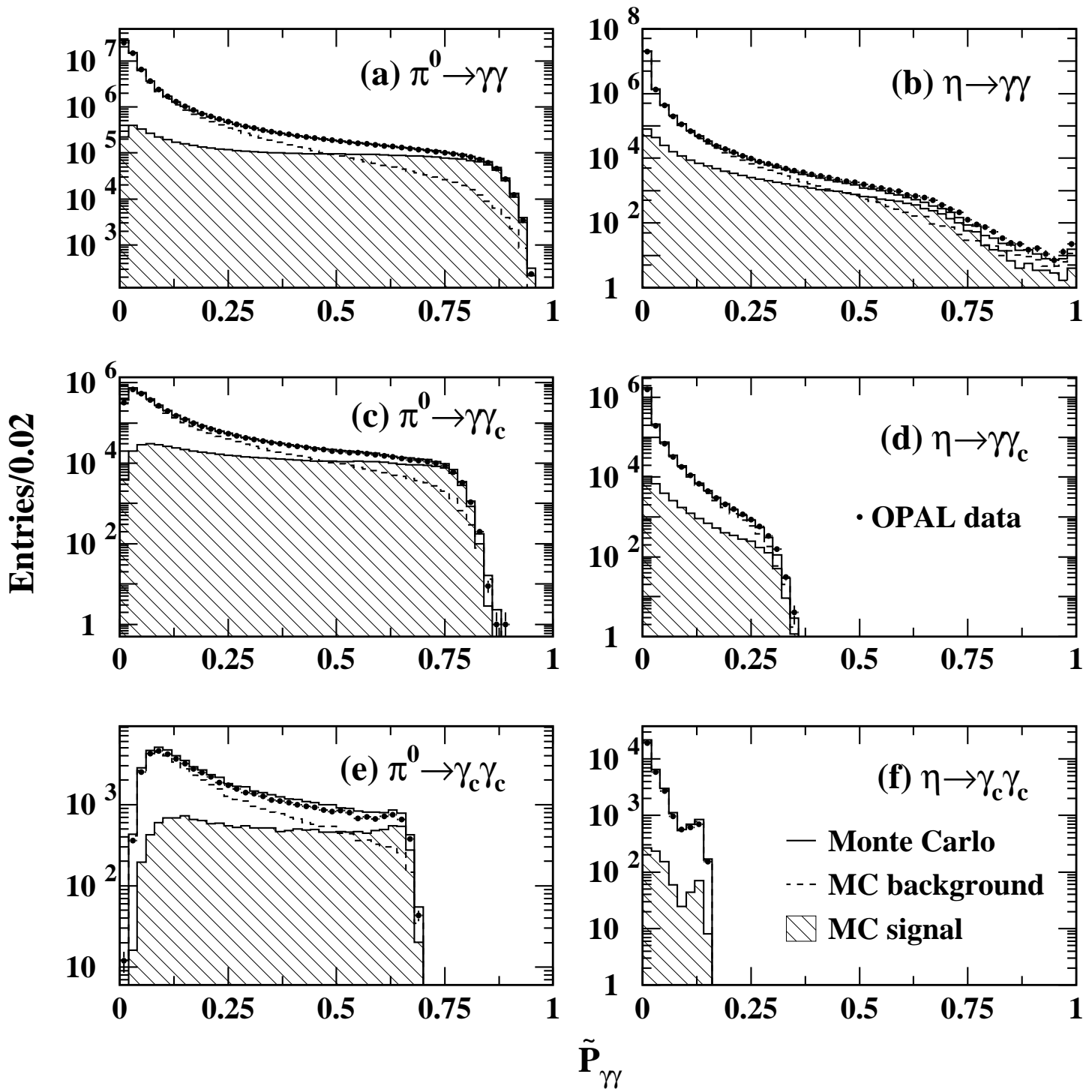

Figure 4: a-f: Distribution of $\tilde{P}_{\gamma \gamma}$ for the channels $\pi^{0} \rightarrow \gamma \gamma, \eta \rightarrow \gamma \gamma, \pi^{0} \rightarrow \gamma \gamma_{c}, \eta \rightarrow \gamma \gamma_{c}$, $\pi^{0} \rightarrow \gamma_{c} \gamma_{c}$ and $\eta \rightarrow \gamma_{c} \gamma_{c}$. The points represent the data and the histograms represent the Monte Carlo simulation normalised to the same number of events. The Monte Carlo signals and backgrounds are shown separately as hatched and dashed histograms, respectively. 


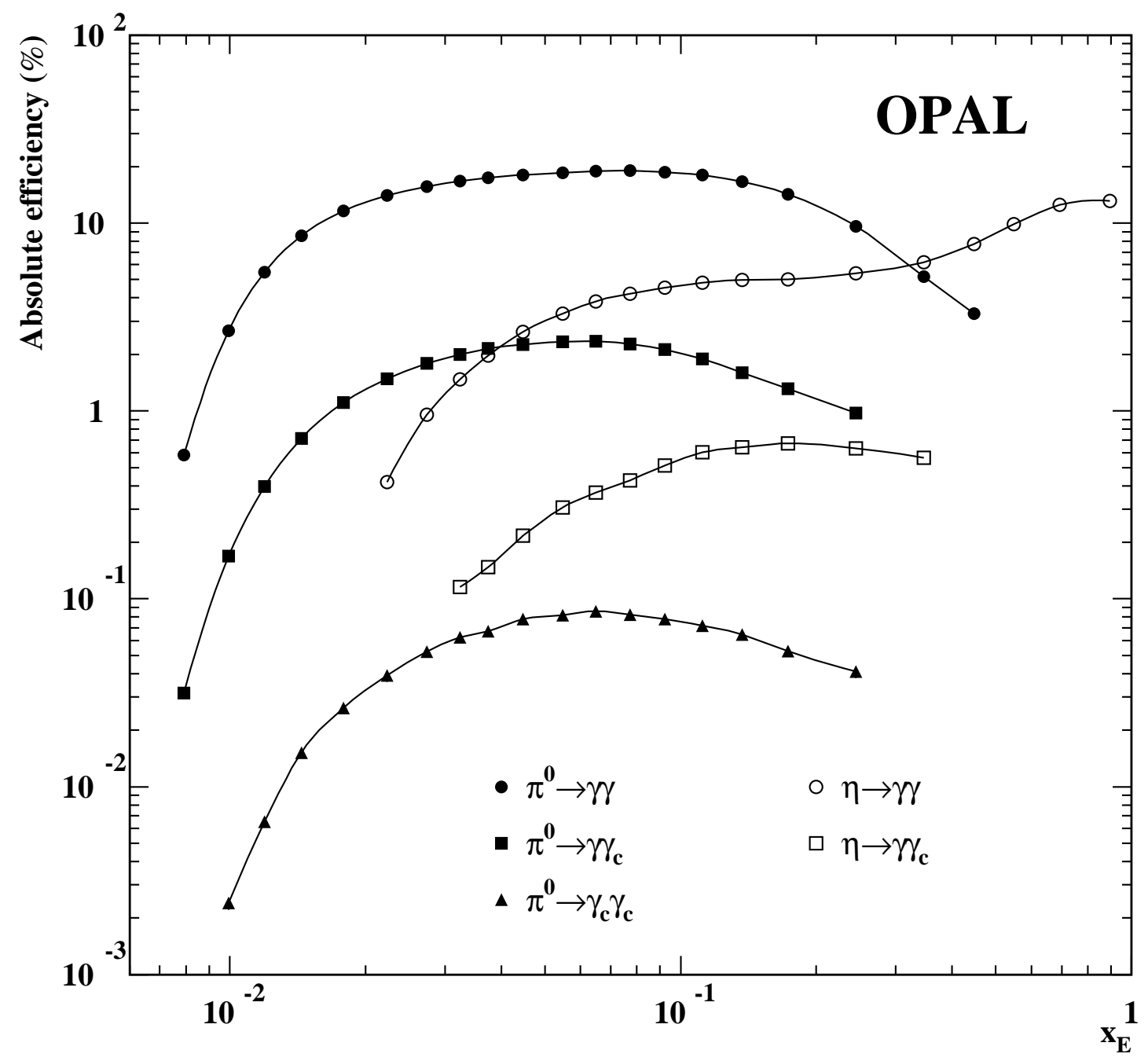

Figure 5: Absolute efficiency as a function of the scaled meson energy $x_{E}$ for the reconstruction and identification of $\pi^{0}$ and $\eta$ mesons, according to the Monte Carlo simulation. The efficiencies for the decay channels $\pi^{0} \rightarrow \gamma \gamma, \pi^{0} \rightarrow \gamma \gamma_{c}, \pi^{0} \rightarrow \gamma_{c} \gamma_{c}, \eta \rightarrow \gamma \gamma$ and $\eta \rightarrow \gamma \gamma_{c}$ are shown separately. A cut on $\tilde{P}_{\gamma \gamma}>0.1$ is applied for the $\pi^{0}$, and $\tilde{P}_{\gamma \gamma}>0.05$ for the $\eta$ (see text). 


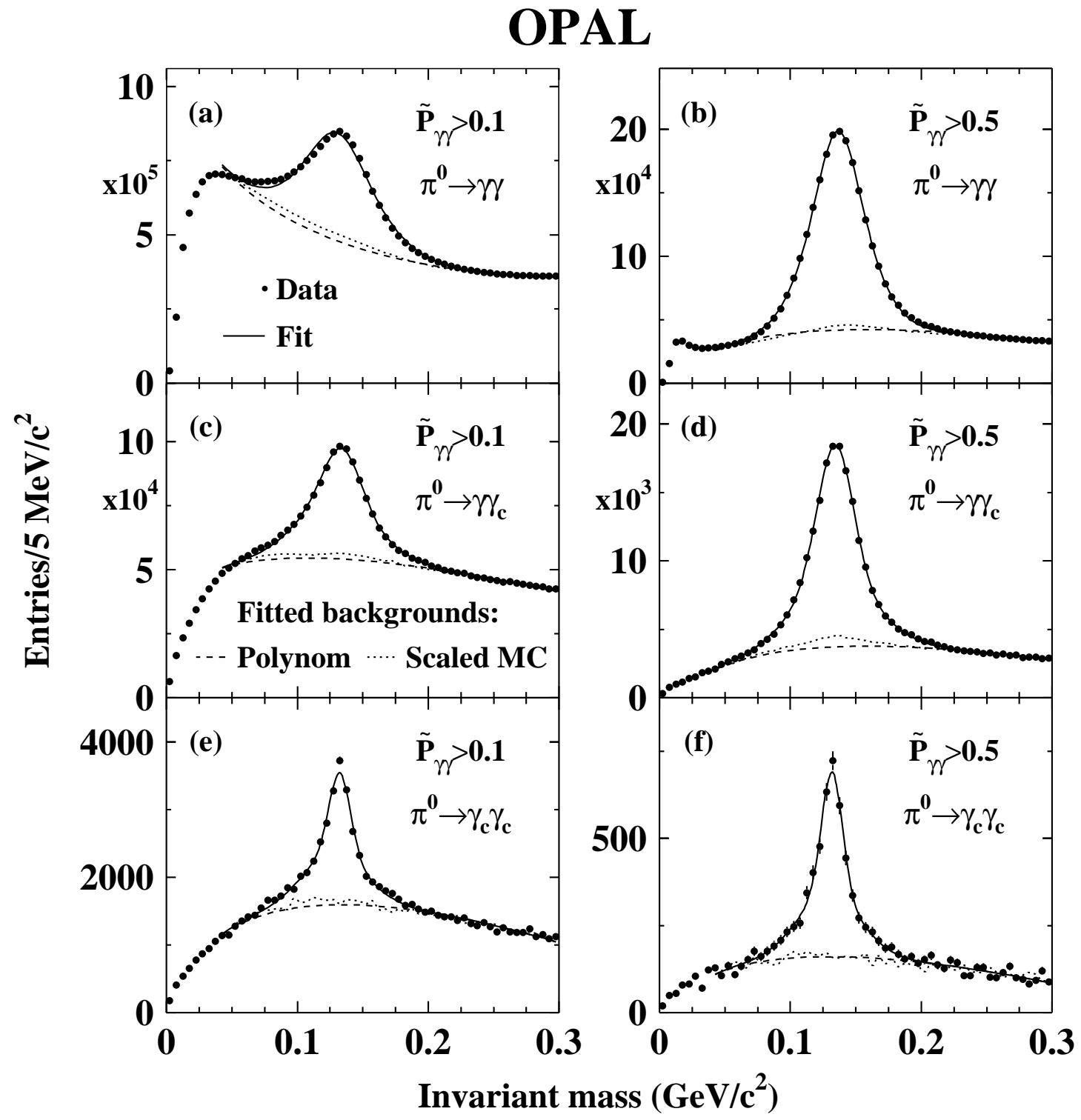

Figure 6: a-f) Fits to the invariant mass distribution of pairs of photons, for the decay channels $\pi^{0} \rightarrow \gamma \gamma, \pi^{0} \rightarrow \gamma \gamma_{c}$ and $\pi^{0} \rightarrow \gamma_{c} \gamma_{c}$ for the lowest and highest $\tilde{P}_{\gamma \gamma}$ cut used in the present analysis. The points represent the OPAL data and the full lines the fits to the data where the background (dashed lines) is parameterized as a second-order polynomial. The dotted lines correspond to the background evaluated using the Monte Carlo distributions, which may contain a small fraction of the signal in cases where the association between photon candidates and true photons is ambiguous (see text). 


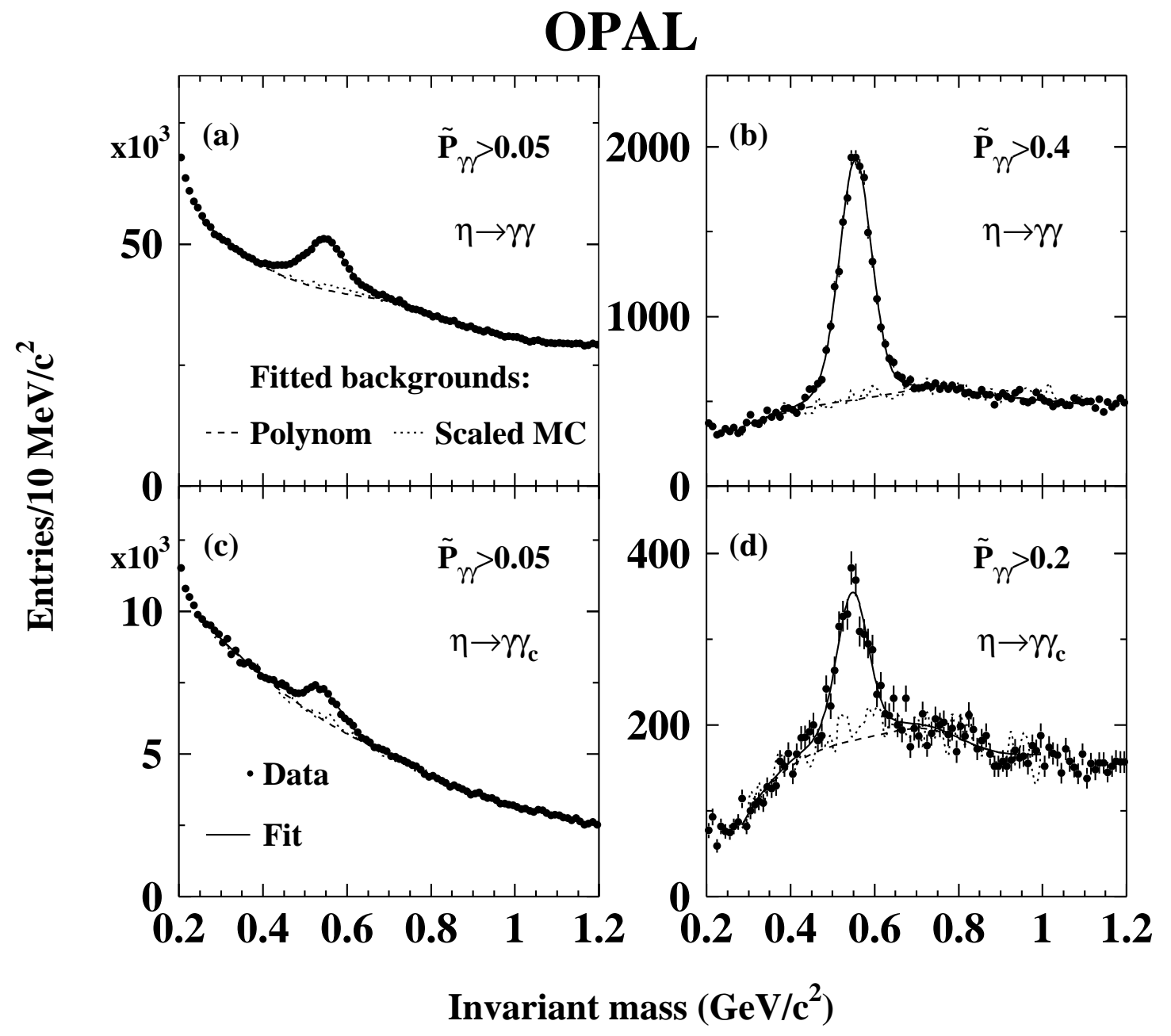

Figure 7: a-d) Fits to the invariant mass distribution of pairs of photons, for the decay channels $\pi^{0} \rightarrow \gamma \gamma$ and $\eta \rightarrow \gamma \gamma_{c}$ for the lowest and highest $\tilde{P}_{\gamma \gamma}$ cut used in the present analysis. The points represent the OPAL data and the full lines the fits to the data where the background (dashed lines) is parameterized as a second-order polynomial plus a Gaussian for the reflection from $\omega \rightarrow \gamma \pi^{0}$ decays. The dotted lines correspond to the background evaluated using the Monte Carlo distributions, which may contain a small fraction of the signal in cases where the association between photon candidates and true photons is ambiguous (see text). 


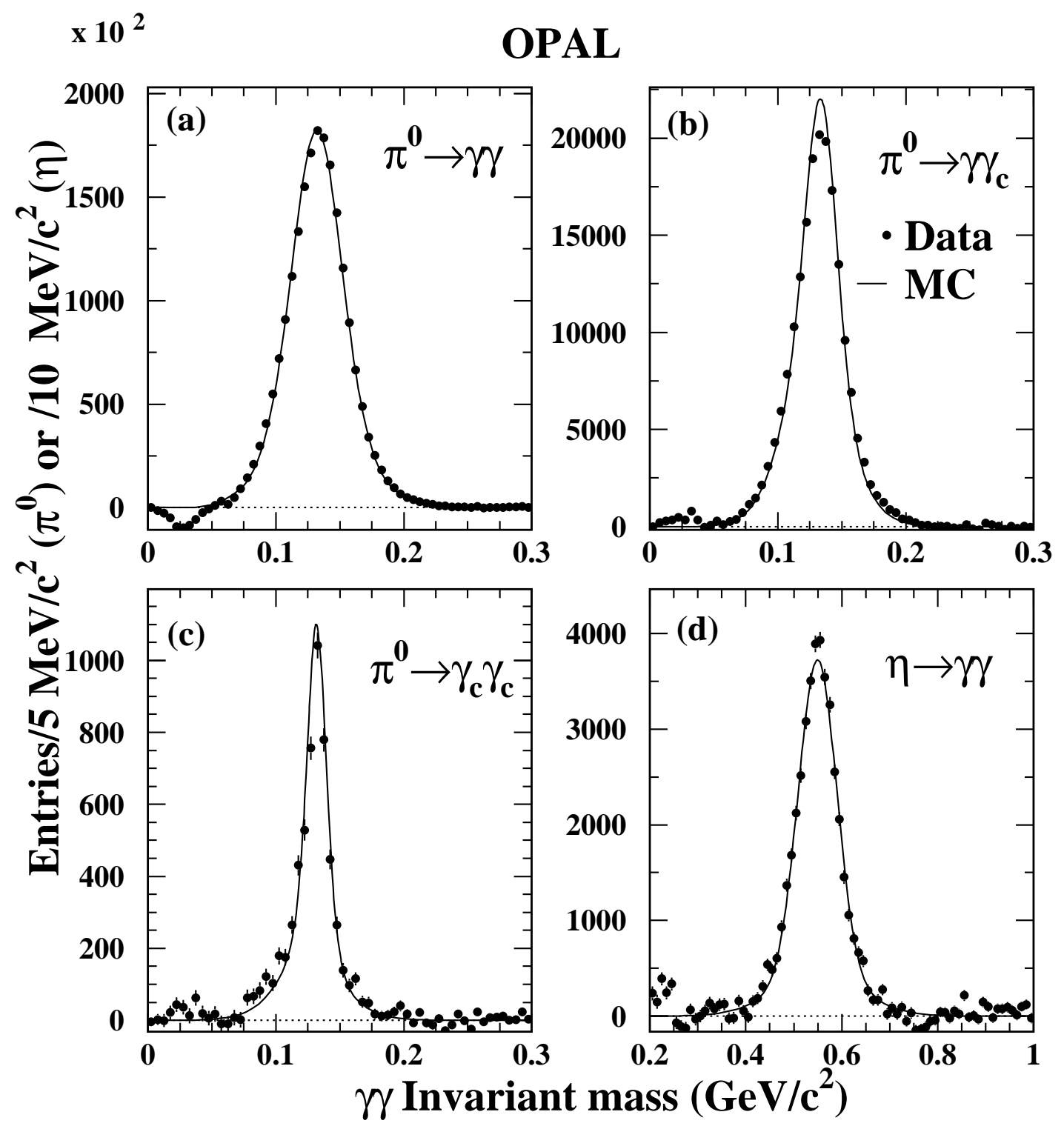

Figure 8: a-d) Mass peak for the decay channels $\pi^{0} \rightarrow \gamma \gamma, \pi^{0} \rightarrow \gamma \gamma_{c}, \pi^{0} \rightarrow \gamma_{c} \gamma_{c}$ and $\eta \rightarrow \gamma \gamma$. The distributions are obtained by subtracting the fitted background shape from the raw spectra. The points represent the OPAL data and the full curves the fit to the Monte Carlo. The Monte Carlo distributions are normalised to the area in the data. A cut on $\tilde{P}_{\gamma \gamma}>0.3$ is applied for the $\pi^{0}$, and $\tilde{P}_{\gamma \gamma}>0.2$ for the $\eta$ (see text). 


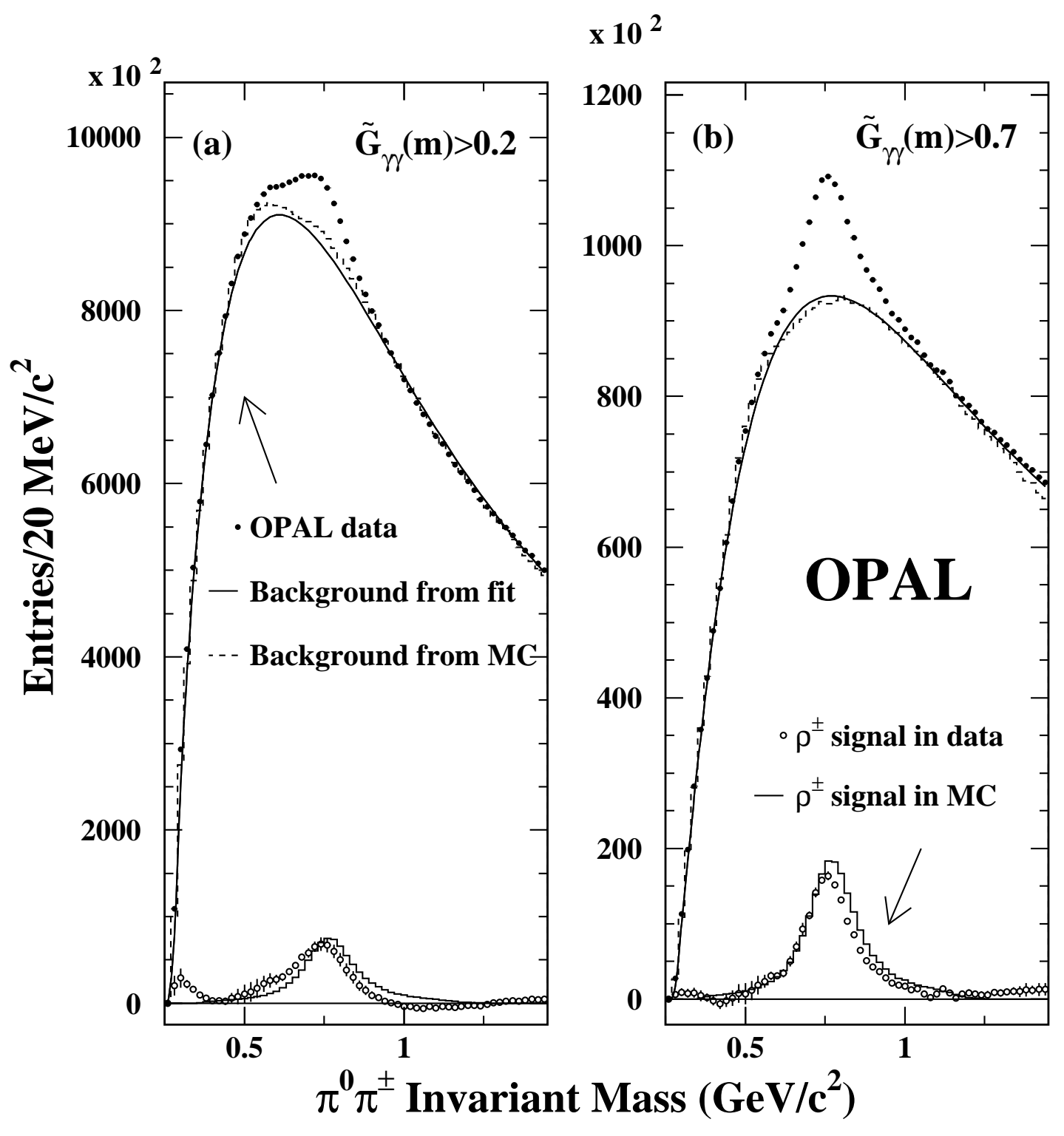

Figure 9: Fits to the invariant mass distribution of $\pi^{0} \pi^{ \pm}$combinations for two different cuts on $\tilde{G}_{\gamma \gamma}(m)$. The points represent the OPAL data and the full curves the background obtained in the fit to the data. The dashed histograms correspond to the background evaluated using the Monte Carlo distributions. In the lower part of the figures, the signal obtained by subtracting the average of the two background from the data is shown as the open circles, and the signal in the Monte Carlo, normalised to the same number of events, is shown as the full histogram. 


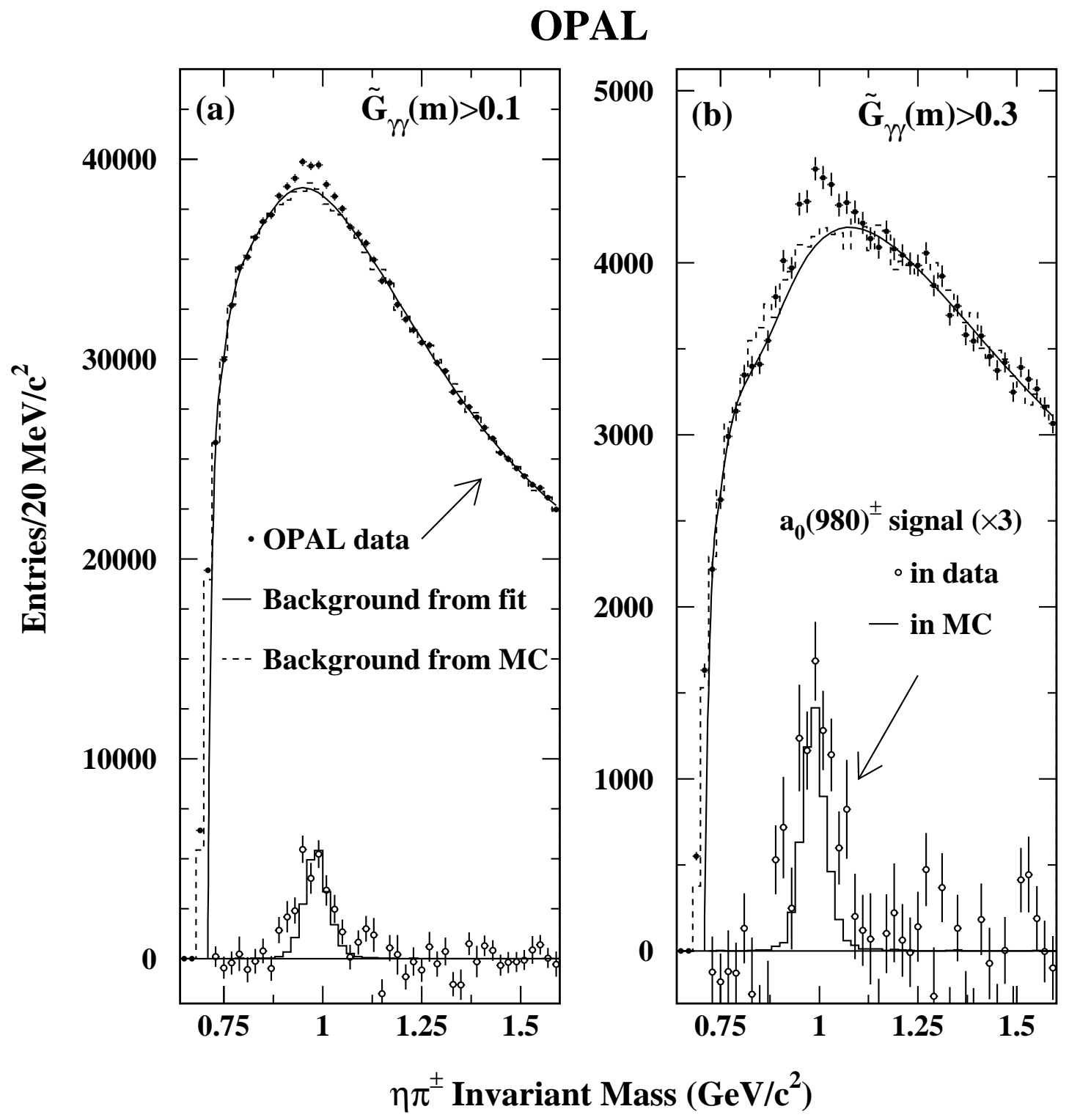

Figure 10: Fits to the invariant mass distribution of $\eta \pi^{ \pm}$combinations for two different cuts on $\tilde{G}_{\gamma \gamma}(m)$. The points represent the OPAL data and the full curves the background obtained in the fit to the data. The dashed histograms correspond to the background evaluated using the Monte Carlo distributions. In the lower part of the figures, the signal obtained by subtracting the average of the two background from the data is shown as the open circles and is multiplied by a factor 3 for clarity. The full histogram is the Monte Carlo signal normalised to the same number of events. 


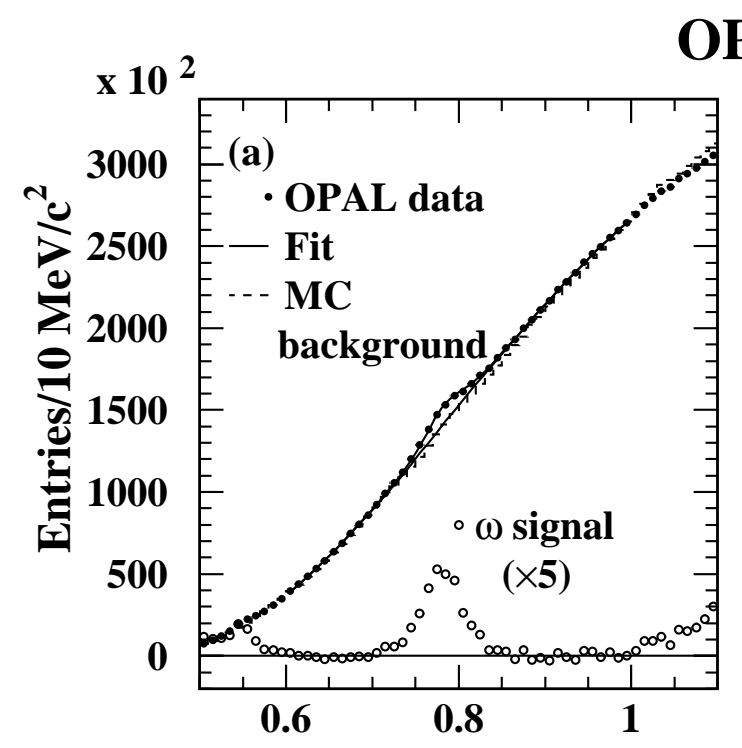

\section{OPAL}
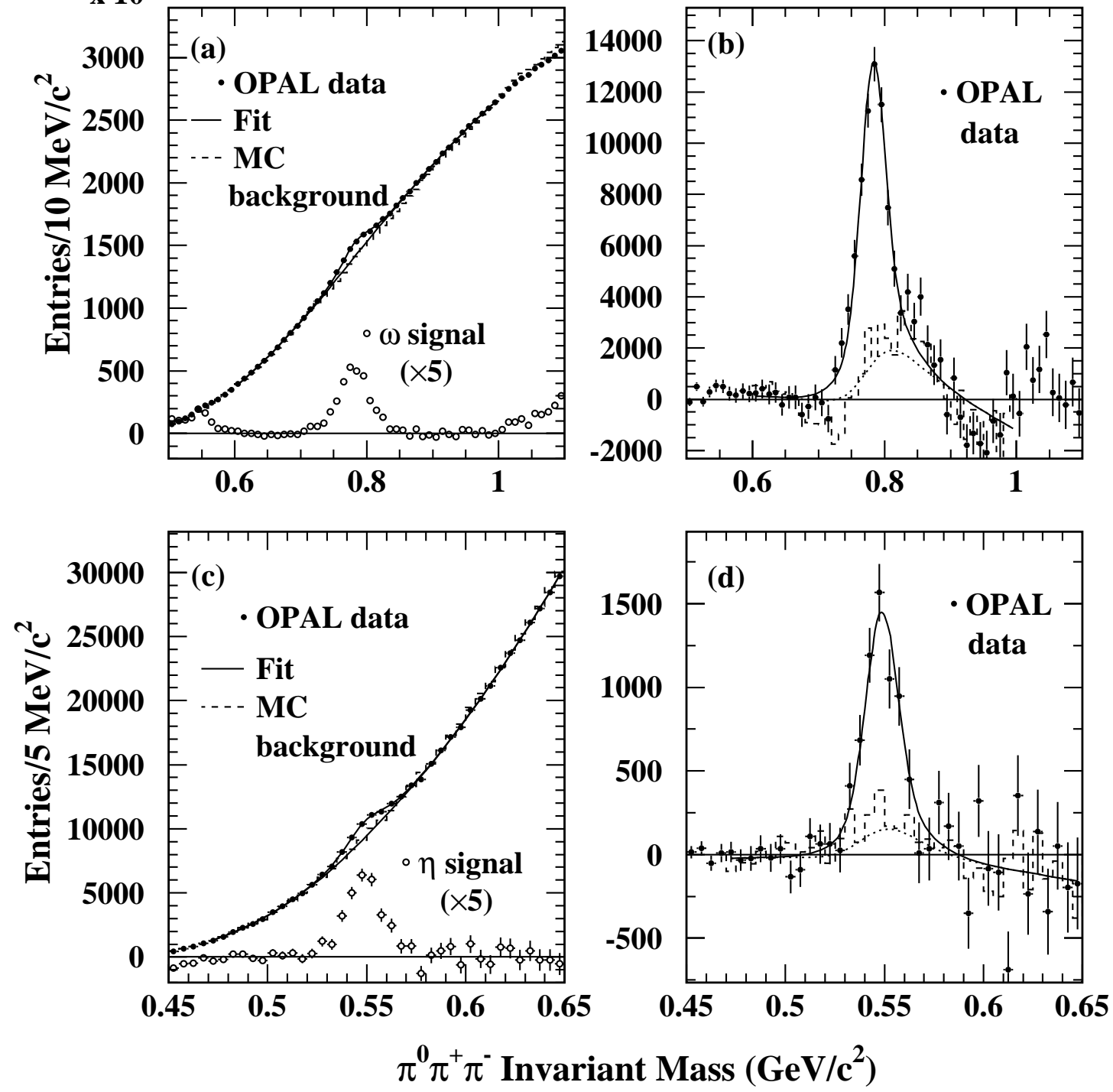

Figure 11: Fits to the invariant mass distribution of $\pi^{0} \pi^{+} \pi^{-}$combinations, for a cut of $\tilde{G}_{\gamma \gamma}(m)>0.3$. The points represent the OPAL data and the full curves the fit to the data. The dashed histograms correspond to the background evaluated using the Monte Carlo distributions. Fig. (a) and (c): fits in the region of the $\omega$ and the $\eta$, respectively. The signal extracted from the fit to the data, multiplied by a factor 5 for clarity, is shown by the open circles. Fig. (b) and (d): the points are the components proportional to $\lambda$ (see section 6.4) extracted from the data in the region of the $\omega$ and the $\eta$, respectively. The full curves are the fits to the data. The dashed histogram is the simulated background, including partially reconstructed mesons which appear as a bump close to the signal peak. The dotted curves are the contributions from partial reconstructions included in the fit. 


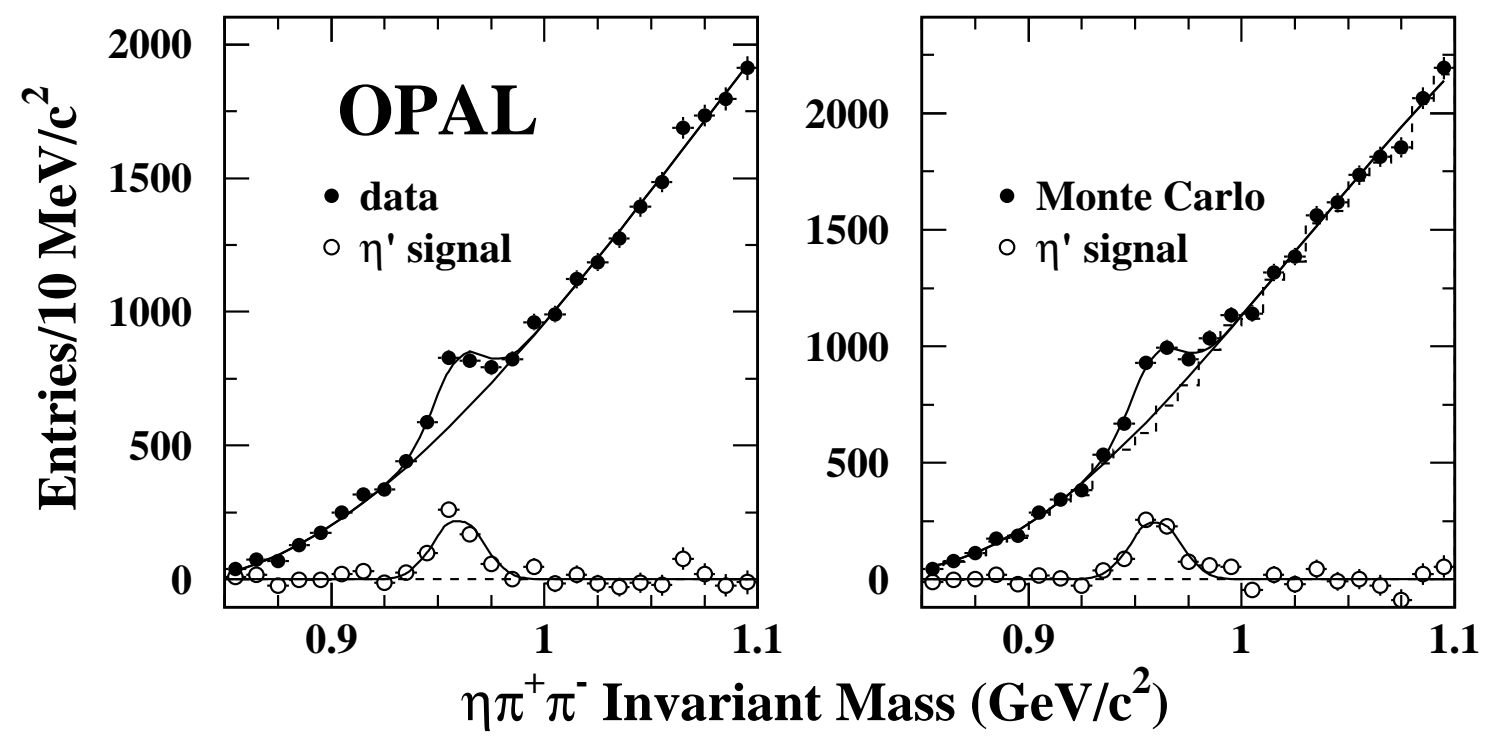

Figure 12: Fit to the invariant mass distribution of $\eta \pi^{+} \pi^{-}$combinations in the data (left) and in the Monte Carlo (right). A cut on $\tilde{G}_{\gamma \gamma}(m)>0.1$ has been applied. The curves represent the fit and the Monte Carlo background is shown as a dashed histogram. In the lower part of the figures, the open symbols represent the signal extracted from the fit. 
OPAL
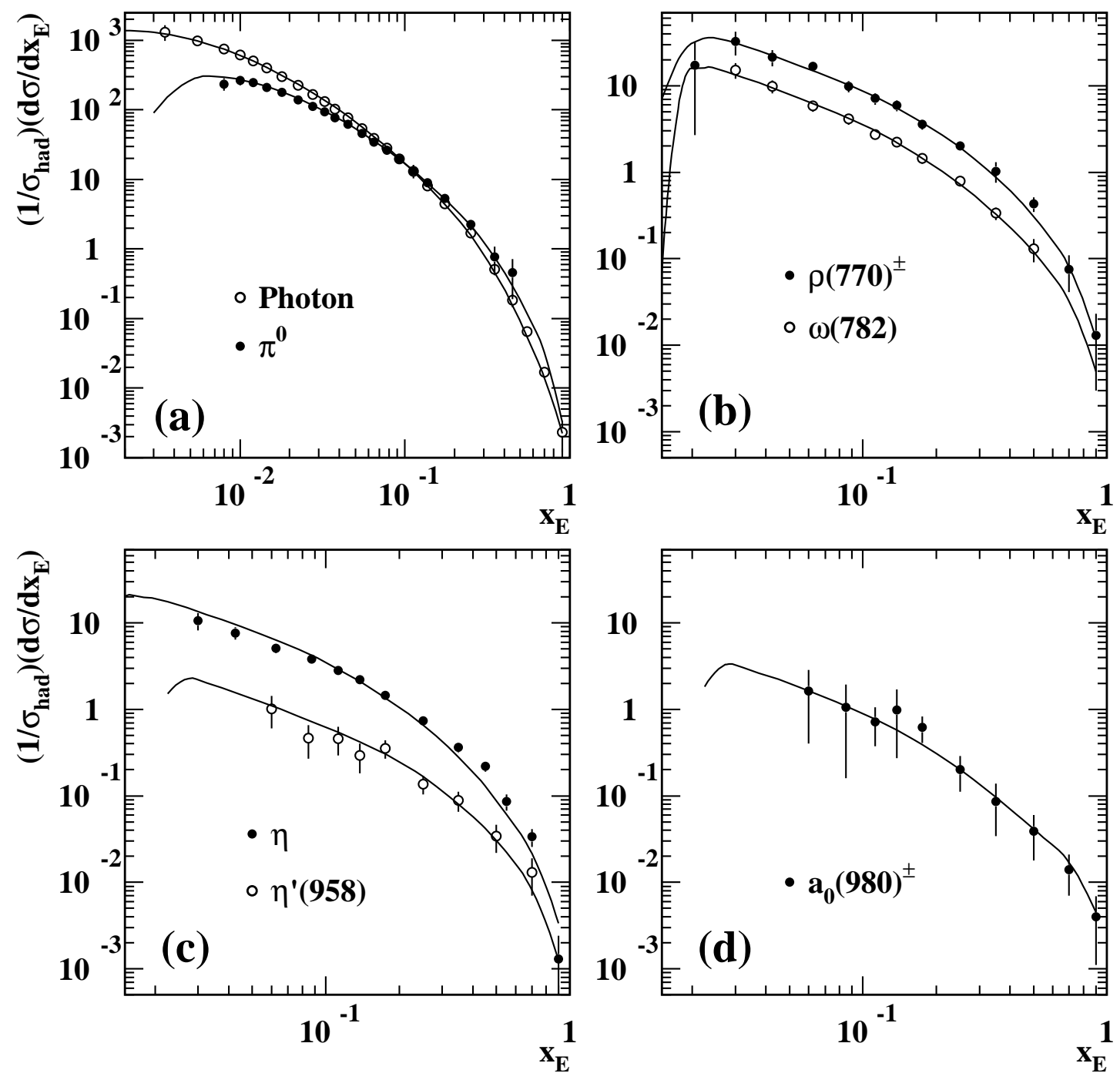

Figure 13: Differential cross-section as a function of the scaled energy $x_{E}$ for the production in hadronic $\mathrm{Z}^{0}$ decays of (a) photons and $\pi^{0}$ mesons, (b) $\rho^{ \pm}$and $\omega$ mesons, (c) $\eta$ and $\eta^{\prime}$ mesons and (d) $\mathrm{a}_{0}^{ \pm}$mesons. The points are the data and the curves are the predictions of default JETSET 7.4, with a normalisation fitted to the data. The error bars represent the quadratic sum of the statistical and systematic errors. 


\section{OPAL}
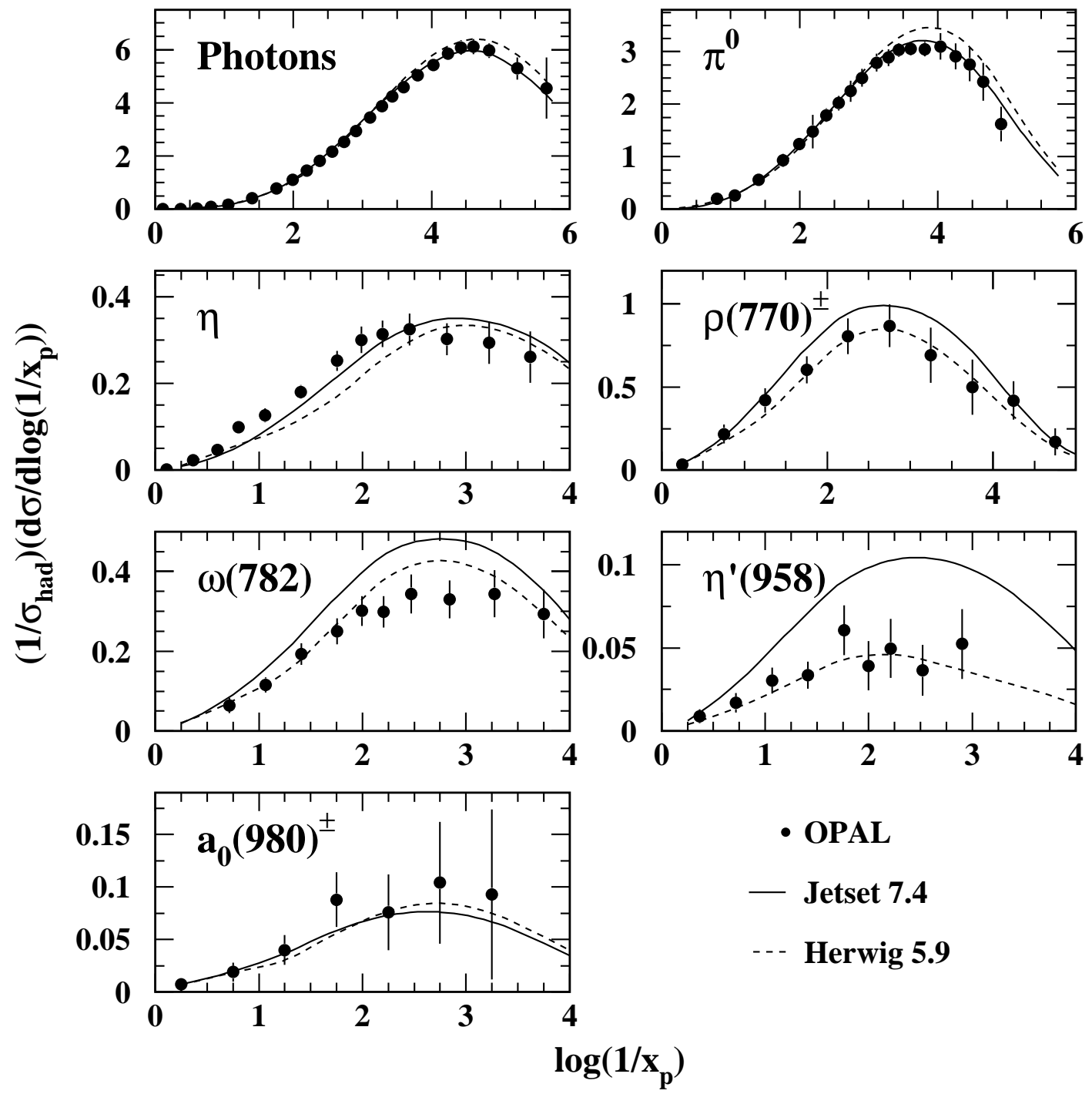

Figure 14: OPAL measurement of the inclusive production of photons, $\pi^{0} \eta, \rho^{ \pm} \omega, \eta^{\prime}$ and $\mathrm{a}_{0}^{ \pm}$mesons in hadronic $\mathrm{Z}^{0}$ decays, as a function of $\log \left(1 / x_{p}\right)$. The full and dashed lines are the absolute predictions of JETSET 7.4 and HERWIG 5.9, respectively, without any additional normalisation. The error bars represent the quadratic sum of the statistical and systematic errors. 

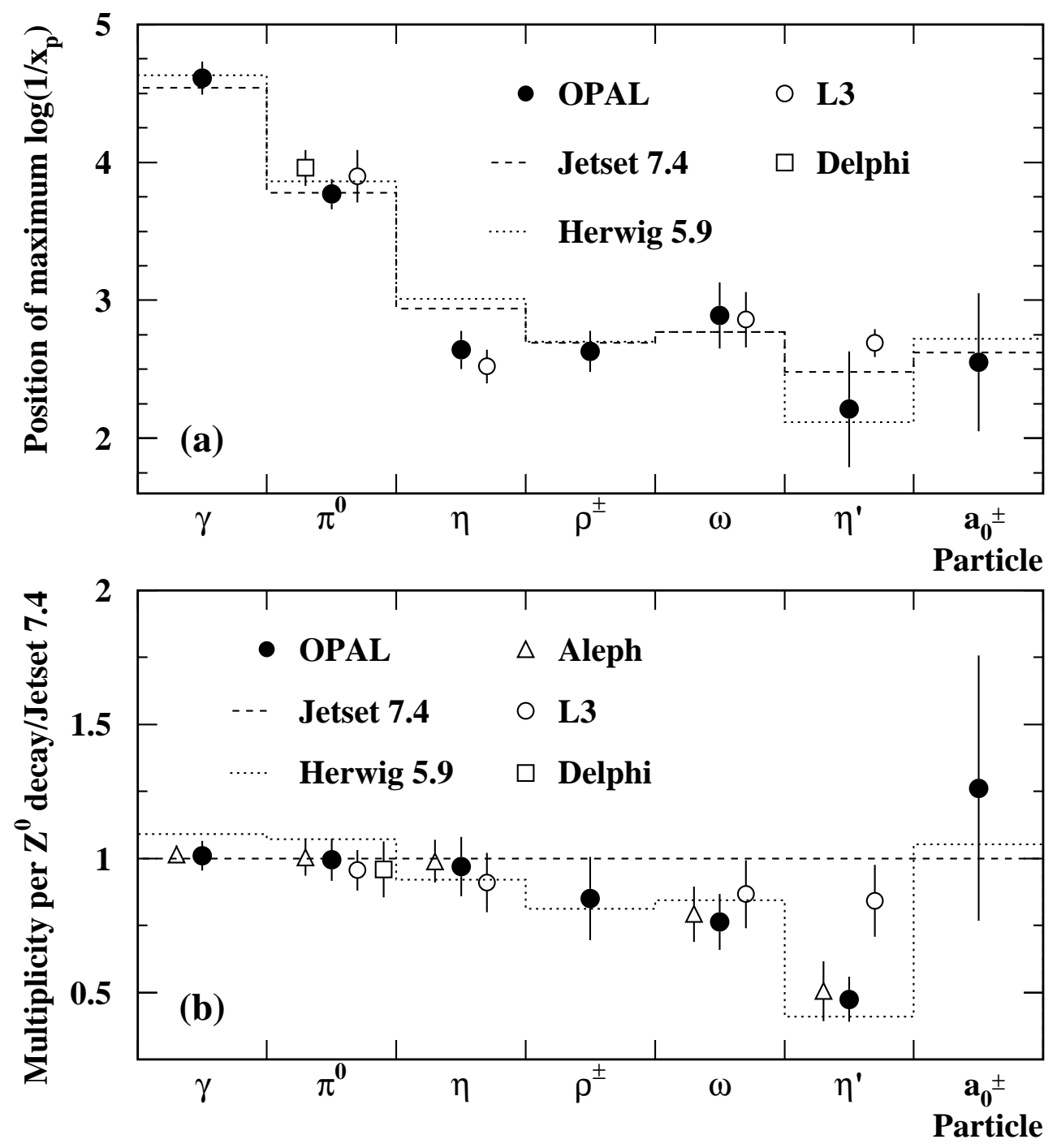

Figure 15: a) Position of the maximum of the $\log \left(1 / x_{p}\right)$ distributions. The black points are the OPAL measurements, and the full and dashed lines are the predictions of JETSET 7.4 and HERWIG 5.9, respectively. The measurements of DELPHI [9] and L3 [10 [12] are shown as white squares and circles, respectively. The maxima are obtained by a Gaussian fit to the data close to the maximum, except for L3, which makes model-dependent assumptions concerning the shape of the distribution. b) Particle multiplicities (extrapolated to $0<x_{E}<1$ ), divided by the prediction of JETSET 7.4. The full circles represent the OPAL measurements, and the open circles, triangles and squares those of other LEP experiments [6, 9 [12]. The dotted line represents the prediction of HERWIG 5.9. 